\title{
Enzymatic Degradation of Egg Yolk Cholesterol
}

A thesis submitted in total fulfilment of the requirements of the degree of Master of Science

by

Speroulla Christodoulou B.Sc.

Victoria University of Technology, Victoria, Australia Food Science Australia, Victoria, Australia

March 1998

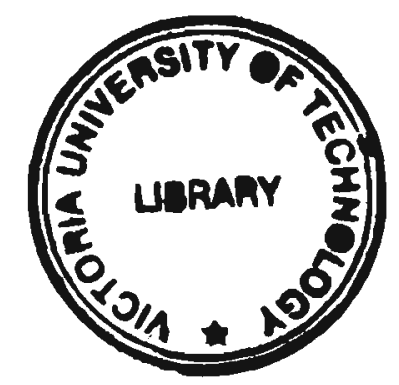




\section{FTS THESIS}

664.024 CHR

30001005475910

Christodoulou, Speroulla

Enzymatic degradation of egg yolk cholesteral 


\section{ACKNOWLEDGEMENTS}

I want to sincerely thank my mother and father for their wonderful encouragement and support, both intellectual and financial, throughout the course of my life, and to gratefully acknowledge the opportunity they have given me to reach a higher level of education.

Special thanks is extended to my supervisors Dr Tran Viet Hung and Mr Robert Black of Food Science Australia and Assoc. Prof. Maurie Trewhella of University of Technology for their invaluable advice, encouragement and expert constructive criticism throughout this work. Particular thanks to Dr Tran Viet Hung and Assoc. Prof. Maurie Trewhella for their patience and persistence.

I would also like to thank LiHui Lui and Milica Rodavanovic, fellow postgraduate students for sharing the experience of postgraduate studies with me, and Gail Di Gregorio of Food Science Australia, for her support and good humour during the sometimes frustrating first year of my thesis. Thanks also to Hein Hamerjylink for providing a much-needed diversion to my studies.

Thanks to Drs Heather Craven and John Coventry and Ms Vivien Boghossian of Food Science Australia, for expert technical advice, and Daphne Wolmersley and Lorraine Ruddick for their helpful assistance.

Many thanks to Mr Kym Butler of the Victorian Institute of Animal Science, for assistance with the design of experiments and statistical analysis, Mr Vince Murone (trouble shooter extraordinaire) of Victoria University of Technology, for assistance with GC-MSD analysis and Dr Harry Laan, post-doctoral fellow from the University of Groningen in the Netherlands, for valuable discussion and to Stella Halkiadakis, Jill Vince, Ian Sullivan and Min Nguyen of the School of Life Science Sector, Victoria University of Technology for their helpful assistance with equipment.

Finally to all my family and friends, whose insistence on running commentaries as to the progress of my thesis at every opportunity secured it's completion, I extend sincere thanks. 
This work was made possible through facilities provided by Food Science Australia and Victoria University of technology and the financial support provided by a grant from the Egg Industry Research Council and a research scholarship from Victoria University of Technology. 


\section{DECLARATION}

The studies described in this thesis have not been previously submitted for any other degree at this or any other University. The studies presented in this thesis represent my own original work except where due acknowledgment has been made. This thesis is less than 100,000 words in length, exclusive of tables, bibliographies, appendices and footnotes.

Speroulla Christodoulou 
For Mum, Dad and my grandparents, $\Gamma \imath \alpha \gamma \alpha$ and $\Pi \alpha \pi o v$ 


\section{CONTENTS}

Page

Abstract

xiii

Publications \& Communications

Xv

List of Abbreviations

xvi

List of Figures and Tables

xviii

\section{CHAPTER 1}

\section{Literature Review}

$1.1 \quad$ Egg Nutritional Value 2

1.2 Egg Composition 2

1.3 Cholesterol Synthesis and Utilisation 4

Transport of Cholesterol in the Bloodstream 5

$1.4 \quad$ Epidemiological Studies and Clinical Trials 7

$\begin{array}{lll}1.5 & \text { Dietary Studies } & 8\end{array}$

Hyper-and Hypo-Responders 9

1.6 Association Between Cholesterol, Cardiovascular and

Coronary Heart Disease $\quad 10$

1.6.1 Health Authority Response 11

1.6.2 Public Response $\quad 13$

Egg Consumption $\quad 14$

1.6.3 Industry Response $\quad 14$

$1.7 \quad$ Low Cholesterol Egg Products-Problem and Approach 15

$\begin{array}{lll}\text { 1.7.1 Selective Breeding } & 15\end{array}$

Species Variation in Egg Yolk Cholesterol Concentration 17

$\begin{array}{lll}1.7 .2 & \text { Diet Modification. } & 19\end{array}$

$\begin{array}{lll}\text { 1.7.3 Pharmaceuticals } & 26\end{array}$ 
1.7.4 Removal of Egg Yolk 28

1.7.5 Removal of Cholesterol-Containing Fat Components 28

Novel Food Ingredients in the Reformulation of Food

Products 29

1.7.6 Extraction Processes $\quad 30$

Steam Distillation 30

Organic Solvents $\quad 30$

Supercritical Fluid Extraction $\quad 32$

Cyclodextrin $\quad 34$

1.7.7 Enzymatic Degradation 36

Industry Significance of Enzymes $\quad 36$

Immobilisation of Enzymes $\quad 38$

$\begin{array}{lll}\text { 1.7.8 Microbiological Degradation } & 39\end{array}$

$\begin{array}{lll}1.8 & \text { Project Aims and Objectives }\end{array}$

\section{CHAPTER 2}

\section{Materials and Methods}

$2.1 \quad$ Cholesterol Assay Conditions 42

Solvents $\quad 42$

Chemicals $\quad 42$

Chromatography Standards $\quad 43$

Instruments 43

Hewlett Packard 5890 Gas Chromatograph 43

Varian 3700 Gas Chromatograph 44

2.1.1 Cholesterol Determination 45

2.1.2 Cholesterol Content in Multi-component Foods 46

$2.2 \quad$ Optimisation of Cholesterol Assay Conditions 46 
$\begin{array}{lll}2.2 .2 & \text { Substrate } & 47\end{array}$

$\begin{array}{lll}2.2 .3 & \text { Silylation } & 47\end{array}$

Silylation reaction Time Studies $\quad 48$

2.2.4 Gas Chromatography $\quad 49$

Positioning of Capillary Column in Injector of Varian

Gas Chromatograph

Relative Response Factors

2.2.5 Reproducibility and Recovery $\quad 50$

2.3 Enzymatic Treatment of Egg Yolk 50

Organic Material $\quad 50$

Enzymes 51

Solvents $\quad 51$

$\begin{array}{ll}\text { Chemicals } & 51\end{array}$

Chromatography Standards $\quad 53$

Egg Sample $\quad 54$

2.3.1 Cholesterol Reductase $\quad 55$

Method of Extraction from Cucumber Leaves 55

Purification and Preparation of XAD-4 Amberlite Resin 56

Purification and Preparation of PVPP 57

Cholesterol Degrading Capacity of Cholesterol Reductase

Enriched Extract $\quad 57$

2.3.2 Cholesterol Oxidase 58

Cholesterol Degrading Capacity Of Cholesterol Oxidase 59

Effect of Time and Temperature 59

Effect of Concentration $\quad 60$

Thin Layer Chromatography 61

Gas Chromatography-Mass Spectroscopy (GC-MS) 61

2.4 Microbiological Treatment of Egg Yolk and Milk 
$\begin{array}{ll}\text { Cholesterol } & 62\end{array}$

Solvents $\quad 62$

Media $\quad 62$

Cultures and Media 63

Growth Curves for Microorganisms 63

Detection of COD Activity 64

Level of Isopropanol Tolerance by Rhodococcus sp. 64

Detection of COD Activity on Agar Medium 64

2.4.1 Cholesterol Degradation in Milk 65

2.4.2 Cholesterol Degradation in Egg Yolk 66

2.4.3 Cholesterol Degradation Products 66

\section{CHAPTER 3}

\section{Optimisation of Cholesterol Assay}

$3.1 \quad$ Introduction

$3.2 \quad$ Results and Discussion

3.2.1 Internal Standards

3.2.2 Silylation

Silylation Reaction Time Study

3.2.3 Positioning of Capillary Column in Varian Gas Chromatograph Injector

3.2.4 Relative Response Factors

3.2.5 Determination of Cholesterol

3.2.6 Reproducibility and Recovery

3.2.7 The Determination of Cholesterol in Multi-component Foods 


\section{CHAPTER 4}

\section{Degradation of Egg Yolk Cholesterol using Purified Enzymes}

4.1

$$
\text { Introduction }
$$

4.1.1 Enzymatic Reactions

4.1.2 Enzyme Specificity

4.2 Conversion of Cholesterol in Food to Coprostanol by Cholesterol Reductase

4.2.1 Introduction

4.2.2 Results and Discussion

Conversion of Cholesterol in Food to Cholestenone by Cholesterol Oxidase

4.3.1 Introduction

Preparation and Characterisation of COD

Specificity and Mode of Action of COD

Cofactors of Cholesterol Oxidase

Cholesterol Oxidation Byproducts

COD Activity and Cholesterol Assay

Potential Application for Cholesterol Degradation

4.3.2 Results and Discussion

Effect of Time and Temperature

Effect of Concentration

Cholesterol Degradation Products 


\section{CHAPTER 5}

\section{Microbial Degradation of Egg and Milk Cholesterol}

5.1

Introduction

5.1.1 Equi Factor.

5.1.2 Cell Activity

5.1.3 Steroid Byproducts

5.1.4 Non-steroid Byproducts

5.2 Results and Discussion

5.2.1 Detection of Cholesterol Degradation Activity in Agar Media

5.2.2 Cholesterol Degradation in Milk

5.2.3 Effect of Culture Growth Medium on the Cholesterol Degrading Activity of Cells in Milk and Egg Yolk

5.2.4 A Comparison of Milk and Egg Yolk Cholesterol Degradation

5.2.5 Products of Cholesterol Degradation in Egg Yolk

\section{CHAPTER 6}

\section{Conclusions}




\section{BIBLIOGRAPHY}




\section{ABSTRACT}

A rapid, reliable and accurate gas chromatographic method for the determination of cholesterol in egg yolk and processed foods was developed from a method of Kovacs (1990). A sample is heated in a sealed tube with a solution of potassium hydroxide in ethanol containing the internal standard, dihydrocholesterol. The sterols are then isolated by solvent extraction, converted to trimethylsilyl (TMS) derivatives and subjected to gas chromatography.

The method was used to determine the cholesterol content of a number of foods in order to demonstrate its application to more complex matrixes. Among egg-based products, Italian egg spaghetti had a lower cholesterol content $(0.14-0.74 \mathrm{mg} / \mathrm{g})$ than egg noodles $(0.87-1.33 \mathrm{mg} / \mathrm{g})$. Some egg rolls contained cholesterol levels as high as $1.72-3.61 \mathrm{mg} / \mathrm{g}$. Among meat-based foods, ham products had cholesterol levels similar to pork cakes or pork loafs $(0.59-0.67 \mathrm{mg} / \mathrm{g}$ and $0.66-0.81 \mathrm{mg} / \mathrm{g}$ respectively). Fish balls and cheddar cheese contained almost the same level of cholesterol $(0.93 \mathrm{mg} / \mathrm{g}$ and $0.95 \mathrm{mg} / \mathrm{g}$, respectively). Of all foods investigated, chicken liver, chicken paste and prawn contained the highest levels of cholesterol.

The capacity of four cholesterol oxidases, from Pseudomonas fluorescens (Pf), Nocardia erythropolis (Ne), Brevibacterium species (Bs) and Streptomyces species (Ss), and cholesterol reductase isolated from cucumber leaves to degrade cholesterol in egg yolk was investigated.

Incubation of egg yolk with a cholesterol reductase for $72 \mathrm{~h}$ at $37^{\circ} \mathrm{C}$ resulted in minimal $(3.6 \%)$ cholesterol reduction. In contrast, up to $93.4 \%$ of the total available cholesterol was degraded by cholesterol oxidases from Pseudomonas fluorescens and Streptomyces species under similar conditions. At $4^{\circ} \mathrm{C}$, cholesterol oxidase from Pseudomonas fluorescens could degrade up to $64.9 \%$ cholesterol 
after $48 \mathrm{~h}$; cholesterol lowering was barely evident at $4^{\circ} \mathrm{C}$ with the other cholesterol oxidases. The most effective temperature was $37^{\circ} \mathrm{C}$ for Bs cholesterol oxidase, and $45^{\circ} \mathrm{C}$ for the other cholesterol oxidases. The enzyme derived from Pseudomonas fluorescens had an effective temperature range from 15 to $60^{\circ} \mathrm{C}$, and from 5 to $37^{\circ} \mathrm{C}$ it was clearly superior to the other three oxidases. The capacity of the cholesterol oxidases to degrade cholesterol in egg yolk followed the sequence Pf $>$ Ne $>$ Ss $>$ Bs. The optimal enzyme concentration for cholesterol degradation was the lowest for Pf cholesterol oxidase at $0.5 \mathrm{U} / \mu$ mole cholesterol. Even at the low concentration of $0.125 \mathrm{U} / \mu$ mole cholesterol, Pf cholesterol oxidase degraded up to a third of the egg cholesterol in $2 \mathrm{~h}$.

The cholesterol degrading activities of Rhodococcus equi, Rhodococcus erythropolis and two other unspeciated isolates of Rhodococcus were investigated in egg yolk and milk. These microorganisms, when grown on a cholesterol containing medium, resulted in halo formation around colonies of the Rhodococcus species consistent with the production of cholesterol degrading enzymes. The ability of Rhodococcus equi No. 23 to degrade cholesterol in whole homogenised UHT milk or preparations diluted to half strength was minimal. However, R. equi No. 23 degraded up to $52.7 \%$ of cholesterol in a $1 / 8$ strength preparation of UHT milk. In these preparations cholesterol degradation was further enhanced by growth of $R$ equi No. 23 cells used as the inoculum in UHT milk rather than in an artificial medium, prior to determination of cholesterol degradation.

The cholesterol degrading system of $R$. equi No. 23 was equally effective in reducing the concentrations of cholesterol in egg yolk. The rate of cholesterol degradation was also enhanced by pre-incubations of $R$. equi No. 23 cells in yolk compared with artificial medium. Cholesterol degradation in egg yolk was almost complete after growth of $R$. equi No. 23 for $7 \mathrm{~d}$ at $37^{\circ} \mathrm{C}$. 


\section{PUBLICATIONS}

The following articles have been published or submitted for publication during the course of this thesis:

Christodoulou S., T. V. Hung, M.A. Trewhella and R. Black (1994) Enzymatic degradation of egg yolk cholesterol J. Food Protection. 57(10), 908-912.

Christodoulou S., T. V. Hung, J. Coventry and M.A. Trewhella Microbial degradation of egg yolk cholesterol Aust. J. Dairy Technol. (submitted).

\section{COMMUNICATIONS}

The following communications have been presented to learned societies:

Christodoulou S., T. V. Hung, M.A. Trewhella and R. Black (1993) Enzymatic degradation of egg cholesterol 26th Convention Proceedings AIFST 2nd-6th May, Adelaide PP.4.

Christodoulou S., T. V. Hung, M. A. Trewhella and R. Black (1993) Cholesterol content of some western foods and oriental analogues X V International Congress of Nutrition 26 September-1st October, Adelaide P31.

Christodoulou S., T. V.Hung, M. A. Trewhella and R. Black (1994) Degradation of milk and egg cholesterol by enzymes and microorganisms 24th International Dairy Congress 18th-22nd September, Melbourne. 


\section{LIST OF ABBREVIATIONS}

\begin{tabular}{ll} 
ABS & Australian Bureau of Statistics \\
Bs & Brevibacterium species \\
BSTFA & N-O-bis-(trimethylsilyl)-trifluoroacetamide \\
CHD & coronary heart disease \\
COD & cholesterol oxidase \\
CPIB & p-chlorophenoxyisobutyrate \\
CR & cholesterol reductase \\
CVD & cardio vascular disease \\
DEAE & 2-(diethyl-amino)ethyl-Sephadex \\
E & enzyme \\
EI & enzyme-intermediate complex \\
ES & enzyme-substrate complex \\
FAD & flavin adenine dinucleotide \\
FDA & Food and Drug Administration \\
FID & flame ionisation detector \\
GC & gas chromatograph \\
GC-MS & gas chromatography-mass spectrometry \\
HDL & high density lipoprotein \\
HMG-CoA & human gonadotrophin CoA \\
HPLC & high performance liquid chromatography \\
JCM & Japan Collection of Microorganisms \\
LDL & low density lipoprotein \\
LRC-CPPT & Lipid Research-Coronary Primary Prevention Trial \\
LSD & least significant difference \\
MSD & mass selective difference \\
MSTFA & N-methyl-N-(trimethylsilyl)-trifluoroacetamide \\
NADH & nicotinamide adenine dinucleotide \\
\hline
\end{tabular}




$\begin{array}{ll}\text { NADPH } & \text { nicotinamide adenine dinucleotide phosphate } \\ \text { NCEP } & \text { National Cholesterol Education Program } \\ \text { Ne } & \text { Nocardia erythropolis } \\ \text { NHFA } & \text { National Heart Foundation } \\ \text { NHMRC } & \text { National Health and Medical Research Council } \\ \text { P } & \text { product } \\ \text { Pf } & \text { Pseudomonas fluorescens } \\ \text { PVPP } & \text { polyvinyl polypyrrolidone } \\ \text { s.e. } & \text { standard error } \\ \text { S/U } & \text { saturated/unsaturated } \\ \text { SFE } & \text { supercritical fluid extraction } \\ \text { Ss } & \text { Streptomyces species } \\ \text { TLC } & \text { thin-layer chromatography } \\ \text { TMS } & \text { trimethyl silyl } \\ \text { U/mmole } & \text { units of enzyme per micromole } \\ \text { UHT } & \text { ultra high temperature }\end{array}$




\section{LIST OF FIGURES AND TABLES}

Figure 1.1 The inputs and outputs that contribute to cholesterol balance. 4

Figure 2.1 The structure of BSTFA (N-O-bis-(trimethylsilyl)trifluoroacetamide), $\mathrm{C}_{8} \mathrm{H}_{18} \mathrm{~F}_{3} \mathrm{NOSi}_{2}$, Alltech (Aust) Pty. Ltd.

Figure 2.2 The structure of MSTFA (N-methyl-N-(trimethylsilyl)trifluoroacetamide), $\mathrm{C}_{6} \mathrm{H}_{12} \mathrm{~F}_{3} \mathrm{NOSi}$.

Figure 2.3 The structure of MSTFA silylated cholesterol, $\mathrm{C}_{27} \mathrm{H}_{45} \mathrm{OSi}\left(\mathrm{CH}_{3}\right)_{3}$.

Figure 2.4 The structure of MSTFA silylated cholesterol, $\mathrm{C}_{27} \mathrm{H}_{47} \mathrm{OSi}\left(\mathrm{CH}_{3}\right)_{3}$.

Figure 2.5 The oxidation of cholesterol to cholestenone catalysed by cholesterol oxidase.

Figure 3.1 Chromatogram showing near baseline separation achieved between dihydrocholesterol (1) and cholesterol (2) under the chromatographic conditions used.

Figure 3.2 Conversion of cholesterol to the TMS ether derivative (Berezkin, 1983), with N-methyl-N-(trimethylsilyl)trifluoroacetamide (MSTFA).

Figure 3.3 The rate of derivative formation in a mixture containing equal amounts of octacosan-1-ol and cholesterol using BSTFA at $100^{\circ} \mathrm{C}$, and gas chromatography traces of the reaction mixtures after (A) $2 \mathrm{~min},(B) 5 \mathrm{~min},(C) 40 \mathrm{~min}$ and (D) $50 \mathrm{~min}$.

Figure 3.4 Gas chromatograms under identical conditions showing (A) peak shape quality of underivatised octacosanol-1-ol and cholesterol, and (B) improved peak shape quality after derivatising with MSTFA.

Figure 3.5 The effect on peak quality by varying the length of capillary column inserted into the injector tube of a gas chromatograph: 
measured by the ratio of peak area between cholesterol and octacosan-1-ol and the peak height of the same two compounds. 80

Figure 4.1 Time course of cholesterol degradation in egg yolk at $37^{\circ} \mathrm{C}$ by the four COD's investigated.

Figure 4.2 Time course of cholesterol degradation in egg yolk at $25^{\circ} \mathrm{C}$ by the four COD's investigated.

Figure 4.3 Time course of cholesterol degradation in egg yolk at $4^{\circ} \mathrm{C}$ by the four COD's investigated.

Figure 4.4 Effect of temperature on the activity of various COD's in egg yolk, after $12 \mathrm{~h}$.

Figure 4.5 Effect of enzyme concentration on the degradation of cholesterol in egg yolk, at $45^{\circ} \mathrm{C}$ after $2 \mathrm{~h}$.

Figure 5.1 Photographs of (A) R. equi No. 23 cells as cocci and (B) $R$. equi No. 23 cells as rods under oil immersion (X1000).

Figure 5.2 Turbidity measurements at $530 \mathrm{~nm}$ of $10 \mathrm{~mL}$ cultures inoculated with $250 \mu \mathrm{L}$ stock culture over time with mechanical shaking, at $30^{\circ} \mathrm{C}$.

Figure 5.3 Halo formation of the four strains of Rhodococcus grown on agar, in the absence (-) and presence ( + ) of cholesterol.

Figure 5.4 Halo formation of the four strains of Rhodococcus grown on agar containing egg yolk.

Figure 5.5 Cholesterol reduction in whole milk and in milk diluted, 1 in 2, 1 in 4 , and 1 in 8 , following with $R$. equi No. 23 at $37^{\circ} \mathrm{C}$ for $7 d$.

Figure 5.6 Time course of cholesterol degradation in (a) yolk and (b) milk, by induced and non-induced cells of $R$. equi No. 23 .

Table 1.1 Egg yolk composition.

Table 3.1 The analysis of cholesterol in egg yolk using either octacosan-1ol or dihydrocholesterol as the internal standard. 
Table 3.2 Comparison of extraction by the modified and original procedures in egg yolk.

Table 3.3 Cholesterol (mg/gts.e.*) content of various foods. 


\section{CHAPTER 1}

Literature Review 


\subsection{Egg Nutritional Value}

Eggs are among the most nutritious foods known, providing a unique, wellbalanced source of nutrients for persons of all ages. They contribute to the body's nutrient needs during rapid growth, and are an excellent food for young children and teenagers. Their high nutrient content, comparatively low caloric value and ease of digestibility make eggs valuable in many therapeutic diets for adults (Cook and Briggs, 1977).

Although eggs contain about $74 \%$ water, they are a rich source of high quality proteins. Experimental nutritionists use egg proteins as a standard for measuring the quality of other food proteins (Cook and Briggs, 1977). Eggs are also an important source of iron, phospholipids, trace minerals, vitamin A, B (including $B_{12}$ ), $E$ and $K$. As a source of vitamin D, eggs rank second only to fish-liver oils. Eggs are low in calcium, however, and contain very little, or no, vitamin C (Cook and Briggs, 1977).

\subsection{Egg Composition}

Whole egg is composed of approximately $64 \%$ white and $36 \%$ yolk (Pratt, 1975). The yolk contains all of the egg lipid; Table 1.1 gives the composition of egg yolk and lists the components of the yolk lipid. 
Table 1.1 Egg yolk composition (Pratt, 1975).

\begin{tabular}{|c|c|}
\hline Component & $\mathrm{g} / 100 \mathrm{~g}$ fresh egg yolk \\
\hline water & 51.1 \\
\hline protein & 16.0 \\
\hline ash & 1.7 \\
\hline carbohydrate & 0.6 \\
\hline lipid & 30.6 \\
\hline triglyceride & 20.0 \\
\hline phospholipid & 8.7 \\
\hline cholesterol & 1.6 \\
\hline cholesteryl ester & trace \\
\hline
\end{tabular}

The fatty acids of the phospholipid are predominantly unsaturated; the main

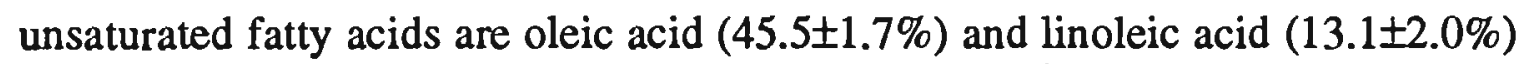
(O'Dea and Sinclair, 1991).

Egg yolk has been described as a concentrated source of cholesterol (Gurr et al., 1989). Reported measurements of egg yolk cholesterol have ranged from 195-270mg/egg (Feeley, 1972; Beyer and Jensen, 1989a; Holden et al., 1989, ).

Most of the cholesterol in egg yolk is found in the low density lipoprotein (LDL) (Gornall and Kuksis, 1971). The composition of LDL in egg yolk comprises of protein (11-17\%), phospholipid (22-28\%) and neutral lipid (59-67\%) (Holdsworth and Finean, 1972).

Egg yolk LDL particles appear to be spheres of lipid surrounded by a layer of glycoprotein, phospholipid and cholesterol with hydrophobic groups on the inside 
and hydrophilic groups oriented toward the outside (Aihara et al., 1988a). Electron microscopy and X-ray diffraction strongly support the concept of a mosaic structure of the surface of the LDL particles in which protein and lipid cover separated areas of the surface and do not form strong associations with each other (Holdsworth and Finean, 1972).

\subsection{Cholesterol Synthesis and Utilisation}

Cholesterol in man is synthesised in many tissues, especially the liver and the small bowel, and is also obtained from the diet (McGilvery, 1979). It is an essential substance in the body, a vital constituent of all cell walls, and is also the precursor of faecal steroids, bile acids and steroid hormones in animals (Lehninger, 1975b). The origin and destination of contributions to the cholesterol balance in the body is shown in Figure 1.1.

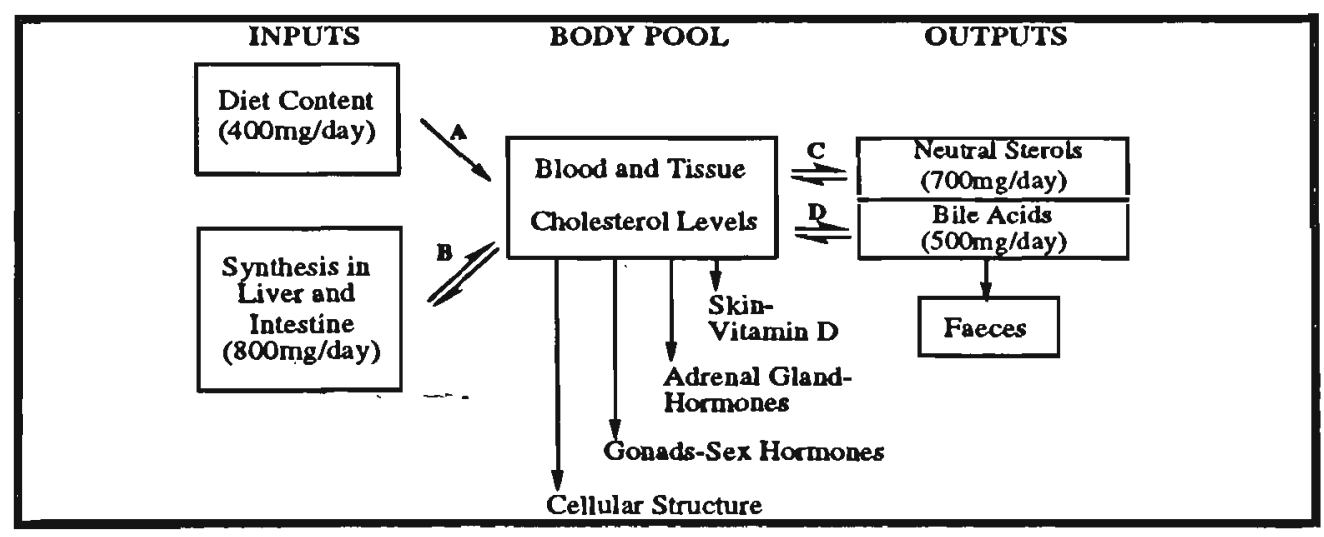

Figure 1.1 The inputs and outputs that contribute to cholesterol balance. Liver and intestinal synthesis in the body normally contribute about twice as much cholesterol to the body pool as does the normal human diet, 800 versus $400 \mathrm{mg}$ per day. Cholesterol is converted in the liver to neutral sterols and bile acids that are released to the intestine and recycled or excreted from the body. Loss in the faeces is usually compensated for by the inputs from diet and synthesis. Changes in dietary cholesterol input would be expected to be compensated for by changes in synthesis to maintain the balance under normal conditions (Naber, 1991). 
Most of the cholesterol synthesised or absorbed from the diet each day is used to replace the bile acids and cholesterol lost in the faeces (McGilvery, 1979). If all systems are functioning optimally the body can adjust cholesterol synthesis and excretion, reducing or increasing each as appropriate to balance cholesterol intake in the diet (O'Dea and Sinclair, 1991). Thus, concentrations of cholesterol can be maintained near a constant level day-to-day, and perhaps month-to-month, despite variations in demand and in dietary supply (McGilvery, 1979).

This physiological feedback system operates in the majority of the adult population, however, in 20-30\% this system does not function (Davidson, 1990). Several human studies have shown that people can be classified into two categories: those with increased plasma cholesterol levels and those with unchanged cholesterol levels, following cholesterol intake (Kestin et al., 1989; Simons et al., 1988).

\section{Transport of Cholesterol in the Bloodstream}

Atherosclerosis is a disease caused by an accumulation of cholesterol in the arteries in the form of bulky plaques that inhibit blood flow and eventually cause a heart attack or stroke (Soehnlen, 1987). LDL is the major cholesterol-carrying lipoprotein in the blood and high levels of LDL can result in the development of atherosclerosis (Soehnlen, 1987). The level of LDL in the blood is affected by specialised proteins called LDL receptors. These receptors bind the LDL particles, which are then taken into the cells where they are broken down, and the cholesterol, which is needed by the cell, is utilised (Brown and Goldstein, 1984). 
Cholesterol is also transported by another lipoprotein, high density lipoprotein (HDL), which has the opposite type of association with atherosclerosis (Nestel $e t$ al., 1989). Elevated levels of HDL-cholesterol, in particular $\mathrm{HDL}_{2}$ particles, are associated with reduced risk of coronary heart disease (Mattson and Grundy, 1985). A significant rise in the $\mathrm{HDL}_{2}$ distribution was reported in men fed two eggs daily, against background diets either high or low in saturated fat, although mean HDL did not change. This may represent a means of clearing dietary cholesterol (Kestin et al., 1989).

The levels of LDL and HDL appear to influence the deposition and accumulation of cholesterol in artery walls, a critical factor in the etiology of atherosclerosis and occlusive vascular disease (Nestel et al., 1989).

An hereditary condition called hypercholesterolaemia causes elevated LDL concentrations. This is as a result of either, an absence or deficiency of the LDLreceptor site protein in plasma membranes, or a failure in the mechanism for endocytosis of the attached LDL from the receptor site (McGilvery, 1979). This disease causes high blood cholesterol and heart attacks in persons under 60 years of age (Soehnlen, 1987) and a high dietary intake of cholesterol could be a contributing factor (Brown and Goldstein, 1984). Therefore, people with a family history of heart attacks or strokes, those particularly susceptible to damaging effects of LDL, should further restrict intakes of dietary cholesterol (Brown and Goldstein, 1984). 


\subsection{Epidemiological Studies and Clinical Trials}

Many different types of epidemiological studies have quantitatively defined the relationship between cholesterol levels and coronary heart disease (CHD) risk (Rifkind, 1985). Studies such as: Framingham, an investigation that helped elucidate the precursors of coronary heart disease (Corday and Corday, 1975); and - Lipid Research Clinics-Coronary Primary Prevention Trial (LRC-CPPT), a double-blinded, primary prevention study of the effect of cholestyramine on men who had hypercholesterolaemia (Gotto and Phil, 1984), have been especially important in establishing the fact that the higher the blood cholesterol, the greater the risk of CHD (Levy, 1985; Rifkind, 1986). The LRC-CPPT findings provided evidence that reduced incidence of CHD is mediated chiefly by total plasma cholesterol and LDL levels (Anon., 1984).

Clinical trial findings are in good agreement with what was suggested by the epidemiological studies, namely, that each $1 \%$ reduction in plasma cholesterol is associated with an approximately 2\% reduction in CHD manifestations (Rifkind, 1986).

A characteristic of many of these trials was the selection of middle-aged subjects suffering from hypercholesterolaemia. Dietary recommendations based on extrapolations made to the rest of the population with less severe elevations of cholesterol, have consequently been regarded by some as unjustified on present evidence (Kronmal, 1985; Ahrens, 1985; Levy, 1985). Nonetheless, these trials have provided some evidence that lowering dietary lipids is beneficial in reducing 
the risk of CHD, and that there exists a possible dose (cholesterol-lowering) response relationship (Rifkind, 1986).

\subsection{Dietary Studies}

Several dietary studies (O'Dea et al., 1991; Kestin et al, 1989b) hrighight a possible correlation between cholesterol levels found in human plasma and background diet. Evidence suggests that it is primarily saturated fat of animal origin (fat in dairy and meat products) that raises cholesterol levels. Polyunsaturated and monounsaturated fats/oils have been shown to have quite different effects on the lipoprotein profile (O'Dea and Sinclair, 1991).

Low-fat diets containing two eggs per day when supplemented with either butter or olive oil resulted in the rise and fall of total plasma cholesterol, respectively. This effect was related to the changes in LDL-cholesterol and HDL-cholesterol levels. Rises in total plasma cholesterol concentration in response to the addition of butter was entirely due to rises in LDL-cholesterol, however, HDL-cholesterol did not change (O'Dea and Sinclair, 1989).

Kestin et al. (1989a) observed no significant change in mean total LDL-cholesterol or HDL-cholesterol plasma concentrations in response to dietary cholesterol when changing from a high fat to a low-fat diet. However, considerable changes did occur in the average size of HDL particles. Proportionately more was present as $\mathrm{HDL}_{2}$, reflecting a compensating mechanism for excess dietary cholesterol through the scavenging potential of HDL (Kestin et al., 1989a). 
A significant rise in serum cholesterol (10\%) was reported when changing from two, to as many as six eggs per week, under well controlled experimental conditions (Beynen and Katan, 1983). In comparison, a rise in serum cholesterol of less than $10 \%$ was measured for a control diet of low cholesterol content (Kestin et al., 1989a). Yet others found no significant elevations in plasma cholesterol concentrations in men ingesting cholesterolequivalent to 2 eggs per week against two background diets (high saturated fat vs fat modified) (Kestin et al., 1989a), suggesting that the influence of dietary cholesterol may be independent of the type of fatty acids present in the dietary fat.

One reason for the discrepancies is the now well established variability in human response to dietary cholesterol (Beynen and Katan, 1985; Simons et al., 1988; Kestin et al., 1989a).

\section{Hyper- and Hypo-Responders}

Hypercholesterolaemic people can be further divided into two groups: hyperresponders and hypo-responders or dietary-sensitive and dietary-insensitive individuals, respectively. Dietary sensitive individuals were found to be more responsive to a background diet low in fat than dietary insensitive individuals when supplemented with cholesterol (Nestel et al., 1989). Hypercholesterolaemic individuals who are sensitive to dietary fat reduction have shown larger responses to dietary cholesterol than either fat-insensitive hypercholesterolaemic subjects or normocholesterolaemic individuals (Kestin et al., 1989a). 
A $3.7 \%$ rise in plasma cholesterol, a $4.9 \%$ rise in LDL-cholesterol and a 5.4\% rise in HDL-cholesterol was seen in hypercholesterolaemic men and women given approximately $700 \mathrm{mg}$ a day of yolk cholesterol in a double-blinded, crossover study while on a background diet containing $25 \%$ of energy as fat, of which $9 \%$ was derived from saturated fat. Normocholesterolaemic individuals experienced small, non-significant rises in total LDL-cholesterol and HDL-cholesterol (Kestin et al., 1989a).

O'Dea and Sinclair (1991) indicated that in normocholesterolaemic subjects the consumption of one egg a day can lower plasma cholesterol, provided the background diet is low in saturated fat (low fat diet or diets containing mono- or polyunsaturated fat). However, these beneficial affects were completely negated when butter was added at a relatively low level (10\% energy). This highlights the important interaction of dietary cholesterol and saturated fat, indicating clearly that if eggs are to be consumed regularly then the saturated fat content of the diet should be kept to a minimum (O'Dea and Sinclair, 1989). Therefore, those suffering from hypercholesterolaemia should use egg yolk in moderation, and normocholesterolaemic subjects need not restrict dietary cholesterol unduly (Kestin et al., 1989a).

\subsection{Association between Cholesterol, Cardiovascular and Coronary Heart Disease}

Coronary heart disease (CHD) and cardiovascular disease (CVD) are leading causes of death for all ages in Australia and North America. Coronary heart disease accounted for half of all deaths in Australia and is responsible for a 
significant proportion of premature deaths in adults (NHFA, 1990). In America, CVD is responsible for more than 550,0000 deaths in the US each year, more deaths than all forms of cancer combined (Consensus conference, 1985). The sense of urgency in research emphasis to isolate the possible causes of CVD and to find a cure has led to a spread of misinterpretations among the public, and even among the scientific community, and has given rise to a controversial issue (Soehnlen, 1987).

Implications made by several epidemiological studies such as the Framingham study (Kannel and Gordon, 1974), the Coronary Primary Prevention Trial (Gordon et al., 1986), the Multiple Risk Factor Intervention trial (Rifkind, 1986) and the Lipid Hypothesis (Soehnlen, 1987), have led health authorities to make dietary recommendations increasing public awareness of the correlations between dietary cholesterol, blood cholesterol and CVD. In fact, these investigations failed to confirm any significant effect of dietary cholesterol on serum cholesterol by either feeding cholesterol (Kestin et al., 1989a) or eggs (Flynn, 1979; Hegsted, 1986; Sidhu, 1989; O'Dea and Sinclair, 1989 and 1991).

\subsubsection{Health Authority Response}

Since epidemiological studies have shown evidence of cholesterol-lowering by cholesterol-lowering drugs or by fat-modified diets, Australians are encouraged to reduce total fat intake, mainly saturated fat, because of its effect on plasma cholesterol. A decline of CHD mortality has been attributed to the increased consumption of polyunsaturated fats in the Australian diet. The energy from 
saturated fatty acids omitted from people's diets has been replaced by complex carbohydrates, monounsaturated and polyunsaturated fats (NHMRC, 1992).

Similarly, in America, the National Cholesterol Education Program (NCEP) has recommended that dietary cholesterol, total fat and saturated fat be limited, both for those at increased risk of CHD and for the general public (Expert Panel, 1988). Based on these factors, the NCEP, American Heart Association and Surgeon General recommend reducing dietary cholesterol to about $300 \mathrm{mg} /$ day (Fairchild, 1991).

A typical Australian consumes about $450 \mathrm{mg}$ of cholesterol per day, about $185 \mathrm{mg}$ of this coming from eggs and dairy fat (Sidhu, 1989). The National Heart Foundation of Australia has provided guidelines recommending the intake of cholesterol-rich foods be reduced and no more than two eggs should be consumed per week due to their cholesterol content (NHFA, 1989).

Therefore, efforts to control serum cholesterol by dietary changes are not limited to the cholesterol in the diet; dietary fatty acid composition has a powerful effect on the cholesterol level in the serum (Keys, 1984). General dietary recommendations outlined by an NHMRC Working Party (NHMRC, 1992) for Australians included reducing the energy intake from saturated fat from $15 \%$ to $10 \%$ of energy. Reducing saturated fatty acids to about $9 \%$ could produce an average reduction of $23 \mathrm{mg} / \mathrm{dL}$ in serum cholesterol (Keys, 1984). 


\subsubsection{Public Response}

In America, the proportion of people believing that a reduction of blood cholesterol would have a large effect on heart disease increased following the release of the results from the Coronary Primary Prevention Trial (72\% of those surveyed, compared with 64\%; Gordon et al., 1986). Dietary changes compared with cholesterol-lowering drugs, were most frequently chosen as ways to control the blood cholesterol level. For instance, reducing dietary fat was believed to be as important as reducing dietary cholesterol. By $1986,23 \%$ of adults reported that they made dietary changes specifically to lower their blood cholesterol level compared with $14 \%$ in 1983 (Schucker et al., 1987a). In a 1996 study by National Family Opinion conducted by the American Egg Board, a consumer concern about cholesterol in the foods they ate further declined to $3.5 \%$, the lowest since 1986 , with $50 \%$ of those questioned citing fats in the diet as the chief culprit of high cholesterol (Anon, 1996).

Before 1983, physicians attributed less preventative value to lowering blood cholesterol than the public, indicative of a reluctance on the part of physicians to make strang endorsements in the absence of conclusive scientific evidence. However, between 1983 and 1986, with the publication of the LRC-CPPT, physicians were more convinced of the benefit of lowering high blood cholesterol levels, and were treating patients accordingly (Schucker et al., 1987b). Elevated blood cholesterol levels were regarded with a level of seriousness approaching that accorded high blood pressure or the risk from smoking, by both practising physicians and the public (Schucker et al., 1987b). 


\section{Egg Consumption}

Dietary recommendations by prominent health authorities based on limited, controversial understanding, coupled with the high level of cholesterol in egg yolk were a likely contributing factor to the decrease in egg consumption experienced by the egg industry. In Australia, the apparent per capita annual consumption of eggs has decreased from 140 to 126 since 1985 (ABS, 1993) and in the U.S. from 303 to 235 during the past twenty years (Elkin et al., 1993).

However, the consumption of processed egg products has continued to increase due to the excellent consumer demand for pre-cooked and convenience products. Perhaps one of the best avenues for increasing per capita consumption of egg is through new processing and product concepts (Larsen and Froning, 1981).

\subsubsection{Industry Response}

Motivated by public concern over cholesterol, the food industry in Australia recognised the public demand for low-cholesterol foods. The Meat and Livestock Corporation market leaner cuts of meat, and the Dairy Corporation now produce low-fat products (Catalyst, 1991).

Like other food organisations, the Egg Industry Research and Development Councils have defined research priorities devoted to the development of technologies for the removal of cholesterol from eggs and egg-based products. 
A market potential of cholesterol-reduced foods including egg-based products is estimated at 25 billion dollars (Hegenbart, 1989). Any reduction in the cholesterol content of market eggs would be valued by both consumers and egg producers (Beyer and Jensen, 1992).

\subsection{Low Cholesterol Egg Products-Problem and Approach}

\subsubsection{Selective Breeding}

Plasma cholesterol levels may represent episodic synthesis and excretion of cholesterol by the liver linked to feed intake or the ovulation period of the hen (Beyer and Jensen, 1992).

The major excretory route for cholesterol is provided by the egg (Sutton et. al., 1984, Sim et al., 1980). Egg yolk cholesterol is important for the developing embryo, and is transferred to a large extent from the yolk to the embryo in the final stages of incubation (Weiss and Scoth, 1979). Increase in excretion of cholesterol via the egg also enables the hen to prevent hypercholesterolaemia when ingesting large levels of dietary cholesterol (Sutton et al.. 1984).

The hen also needs to synthesise cholesterol for use as structural components of cell membranes, as a precursor to sex and adrenal hormones, vitamin D and the bile acids (Hargis, 1988).

Attempts to produce a low cholesterol egg by selective breeding and dietary modification, have been based on the theory that plasma cholesterol levels in the 
hen have a direct influence on those found in the yolk. However, these attempts have yielded only moderate results, adding credence to the alternative theory that the concentration of plasma cholesterol in the hen is not closely associated with that found in the egg (Weiss et al., 1967b, Washburn and Nix, 1974b, Sutton et al., 1984).

Eggs collected from nineteen producers varied in cholesterol content, according to egg weight, yolk weight, hen age, egg production and dietary protein (Beyer and Jensen, 1989a). Eggs from broiler breeders contain higher concentrations of cholesterol than eggs from commercial layer strains (Turk and Barnett, 1971) and moulted flocks have a higher level of total cholesterol than non-moulted flocks due to larger egg size (Beyer and Jensen, 1989a). These studies determining the effects of various parameters on egg cholesterol levels confirm the possibility of genetically selecting for decreased egg yolk cholesterol (Bair and Marion, 1978).

Eggs from seven inbred lines of chickens showed significant differences in yolk cholesterol (Bair and Marion, 1978). Other experiments indicate that strain of commercial layer is not a factor affecting cholesterol levels (Beyer and Jensen, 1989a), as (1) no significant differences in total egg cholesterol or cholesterol concentrations in eggs have been found between commercial egg layer strains (Turk and Barnett, 1971), and (2) significant differences in yolk cholesterol have been seen in the same strain at two different ages (Bair and Marion, 1978).

These findings agree with those of Turk and Barnett (1971) who found considerable variation among individual eggs from the same flock in the concentration of cholesterol as well as in the total amount of cholesterol per egg 
regardless of the age, feed, management or strain. They concluded that there were not enough differences in the amount of cholesterol in the eggs laid by birds of several commercial strains to provide a basis for choosing one strain over another.

These variations, even among birds of the same strain, indicate the difficulties for breeders to select for, and maintain, low cholesterol egg yolk concentration. Many of these variables become interrelated with the passage of time, and this may cause difficulties for breeders when trying to maintain low cholesterol egg yolk concentration in selected strains.

Genetic differences between different strains of chickens may possibly result in differences in cholesterol metabolism (Kondra and Hodgson, 1961; Washburn et al., 1975). However, birds chosen for high and low oxygen-consumption, which theoretically alters metabolic rate, exhibited altered rates of cholesterol and lipid synthesis, but genotype did not appear to alter the minimum level of cholesterol maintained in the egg (Sutton et al., 1984). Genetic selection to develop a chicken line which could produce low cholesterol eggs has only yielded moderate results (2.4\% reduction) (Hargis, 1988; Naber and Biggert, 1985).

\section{Species Variation in Egg Yolk Cholesterol Concentration}

Variation has been found among various species of birds in the concentration of cholesterol as well as in the total amount of cholesterol per egg. Significant differences were found between domestic and wild genetic groups for turkeys and ducks (Bair and Marion, 1978). Eggs from turkey, duck and quail contain higher concentrations of cholesterol than chicken eggs (Turk and Barnett, 1971), 
therefore a shift in consumption from chicken to turkey, duck or quail groups would not reduce dietary cholesterol.

In conclusion, cholesterol levels in the hen result from a balance of dietary intake, biosynthesis, and excretion of cholesterol and cholesterol by-products (Sutton $e t$ $a l ., 1984)$ with yolk cholesterol concentrations for a given hen remain relatively constant (Bartov and Reiser, 1973). Even the age effect whereby total egg cholesterol increased as birds aged did not alter the concentration of yolk cholesterol, the observed effect was a result of increased egg size (Turk and Barnett, 1971).

The moderate results obtained through genetic selection (Hargis, 1988; Naber et al. 1976; Marks and Washburn, 1977), indicate that there may be a low heritability for low-cholesterol concentration. Selection for low cholesterol levels has usually only been effective in the upper cholesterol range (Marks and Washburn, 1977), again suggesting the importance of a basal level of cholesterol required for egg formation (Sutton, 1984).

The increased efforts required to decrease yolk-cholesterol by this approach would undoubtedly be expensive (Bair and Marion, 1978). A more economical approach for producing low cholesterol eggs could be through modification of the hen's diet. 


\subsubsection{Diet Modification}

The cholesterol content of egg yolk has been found to be affected by a number of factors including dietary supplements (Washburn and Nix, 1974b). The amount of cholesterol biosynthesis by the liver (Weiss et al., 1967a) has been shown to vary significantly with dietary treatment (Vargus et al., 1986). Some of the dietary factors identified as influencing yolk cholesterol are the addition of the plant sterols ( $\beta$-sitosterol and soy sterol), the quantity and type of fatty acids present and the type and quantity of fibre present (McDonald and Shafey, 1989).

Plant Sterols The inclusion of sterols, other than cholesterol in the diet has been shown to reduce yolk cholesterol (McDonald and Shafey, 1989).

Plant sterol supplemented diets had no effect on egg cholesterol levels, but diets supplemented with corn oil, or corn oil and plant sterols, showed small, but significant increases in the level of egg cholesterol. Chickens bred from hens fed a commercial low-fat laying diet supplemented with $4 \%$ plant sterols, with or without $10 \%$ corn oil, had significantly reduced levels of egg cholesterol (up to 50\%) (Konlande and Fisher, 1969; Kudchodkar, 1976). This was thought to be related to cholesterol absorption (Kudchodkar, 1976), the ability to absorb dietary cholesterol being highly dependent upon the nature of dietary oil (Sim and Bragg, 1978; Sim et al., 1980).

Sitosterol Standard rations supplemented with $2 \%$ or $4 \%$ sitosterol emulsion reduced egg cholesterol by as much as $35 \%$. Maximal effects were obtained at high concentrations of sitosterol fed for longer periods (Clarenburg et al., 1971). 
Sitosterol acts by interfering with the intestinal absorption of dietary enterohepatically circulating cholesterol, thus, lowering cholesterol levels by promoting faecal excretion of sterols and their degradation products. This suggests that the inclusion of adequate amounts of sitosterol in an absorbable form in the standard diet of laying hens would profoundly affect the egg sterol content (Clarenburg et al., 1971). It has been postulated that the presence of sitosterol in egg yolk (3.8\%) may also depress intestinal absorption of the reduced egg cholesterol content by the human consumer (Clarenburg et al., 1971).

A conflicting report by Kudchodkar et al. (1976), in which hens were fed a commercial low-fat diet supplemented with $\beta$-sitosterol, provided little evidence that sitosterol passed through the intestinal barrier. Up to $93.4 \%$ of fed $\beta$ sitosterol was recovered in the faeces, with up to $80 \%$ of fed cholesterol being absorbed. A similar effect was seen when hens were fed the same diet with the inclusion of $10 \%$ corn oil and $4 \%$ plant sterols. The effect of feeding sitosterol on egg cholesterol levels was insignificant and unless hens were fed high $\beta$-sitosterol diets there was no evidence of $\beta$-sitosterol in the eggs (Kudchodkar et al.s 1976). Kudchodkar et al., (1976) reported that $2.2 \%$ of the absorbed dose of $\beta$-sitosterol was deposited in eggs laid by hens fed high $\beta$-sitosterol diets, compared to the 3.8\% observed by Clarenburg et al. (1971).

Soy Sterols Sim and coworkers reported that reduction of yolk cholesterol levels between 16 and 33\% resulted when hens were fed soy sterols combined with various saturated and unsaturated oils (Sim and Bragg, 1978; Sim et al, 1980). These workers suggested that phytosterols produce their effect through enhanced rate of cholesterol turnover and excretion as bile. 
Grains A significant reduction of $10 \%$ in the level of egg yolk cholesterol was observed when triticale was substituted for wheat, however, a significant decrease could not be demonstrated when either barley or maize was substituted for wheat (McDonald and Shafey, 1989).

Garlic and Lucerne McDonald and Shafey (1989) found that when hens were fed fish oil and garlic mixed with some wheat, up to a $7 \%$ reduction in egg cholesterol was achieved. However, others found no influence on egg cholesterol concentration on modifying the hen's feed either by dietary lipid treatment or by the addition of garlic oil (Reddy et al., 1991, Watkins and Elkin, 1992).

Significant reductions in yolk cholesterol were obtained following the addition of lucerne meal to a maize-based diet (McDonald and Shafey, 1989). However, the addition of lucerne and $\alpha$-ketoisocaproic acid (KIC) to laying hen diets failed to consistently reduce egg yolk cholesterol levels.

Fatty Acid Content Various attempts have been made to reduce the cholesterol level in the egg by modifying the fatty acid content in the hen's diet. In Australia fat-modified eggs called Veggs are marketed. These eggs contain less saturated fat as a result of feeding hens a specially formulated fat modified natural vegetarian diet. The replacement of oleic acid in the egg with linoleic acid is facilitated by vegetable oil feeding with the result that the lipids in the egg yolk become more unsaturated. Feeding hens high fat diets may result in inflated cholesterol by stimulating the synthesis of cholesterol in the liver and deposition in the egg (Washburn and Nix, 1974a). 
The addition of unsaturated oils to the diet has consistently increased yolk cholesterol (McDonald and Shafey, 1989). Beyer and Jensen (1989b) found the addition of animal by-products (mostly meat meal) to the hen's diet, as a source of dietary cholesterol, did not increase the cholesterol content of eggs, compared to those flocks which were not fed animal by-products.

The presence of highly unsaturated fatty acids in the diet increases the absorption of dietary cholesterol and increases the yolk cholesterol while saturated fatty acids have little effect (Sim and Bragg, 1978). Part of the effect of unsaturated fatty acids may be related to fat and cholesterol transport from the liver. Phospholipid synthesis has been shown to be enhanced by unsaturated and unaffected by saturated fatty acids (Hargis, 1988).

No change in egg cholesterol content was observed by Edwards et al. (1962) upon feeding diets containing either corn oil, beef tallow, or lard at a level of $10 \%$. Combs and Helbacka however, observed an increase in the cholesterol content of egg yolk upon feeding a diet containing corn oil at a level of $10 \%$, but not when they fed a diet containing animal tallow at the same level (Weiss et al., 1964).

Chicks exhibit an increased turnover rate of plasma cholesterol when fed a high protein diet. This is associated with an increased faecal excretion of neutral steroids and bile acids (Yeh and Leveille, 1972). When fed a low-fat laying diet, total faecal steroids is much lower than that contained in the egg, however, when diets are supplemented with unsaturated fats, faecal steroid excretion can increase to levels close to those found in the egg (Sim et al., 1980). 
Dietary Fibre Dietary fibre influences cholesterol metabolism by (1) decreasing absorption of dietary cholesterol, (2) binding with bile salts in the intestinal tract, (3) shortening intestinal transit time and (4) increasing faecal excretion (McDonald and Shafey, 1989).

Yolk cholesterol concentration may be reduced by as much as $15 \%$ by choosing a suitable source of fibre. Inclusion of fibre in the diet also results in significant changes in the levels of some nutrients which may affect cholesterol metabolism, eg., changes in the level and type of fat, metabolisable energy or phytosterols (McDonald and Shafey, 1989).

Additives such as cellulose, alfalfa meal, oat hulls, cellulose and pectin or DEAE Sephadex to diets, based on either wheat and soybean meal or corn and soybean meal, were investigated (Turk and Barnett, 1972). Alfalfa added to corn-soy rations was the most effective of the fibre sources tested by Turk and Barnett (1972) with least loss of egg size, feed efficiency and egg production. The DEAE sephadex additive resulted in slightly lower egg cholesterol content than found when a conventional corn-soy layer ration was fed.

Feeding alfalfa meal, oats, sunflower meal, rice mill feed, or wood shavings to laying hens significantly decreased egg cholesterol levels when compared to those of hens fed a basal diet of corn-soybean meal. The greatest reduction was reported on feeding either oats or wood shavings up to $2 \%$ added fibre (McNaughton, 1978). This was achieved without adversely affecting either egg production or egg weight. 
Oat hulls and pectin most effectively reduced cholesterol in wheat diets, but also decreased feed efficiency and egg size (Turk and Barnett, 1972). When corn diets that included DEAE sephadex were fed, egg production, feed efficiency, and egg size tended to be improved, and cholesterol concentration and cholesterol per egg were lower (Turk and Barnett, 1972). When sunflower meal was used as the fibre source a linear decrease in egg cholesterol was correlated with increasing levels of the dietary fibre (McNaughton, 1978). Leghorn pullets fed on basal layer ration containing $30 \%$ oat bran and $3 \%$ cotton seed hulls lowered $(\mathrm{p}<0.05)$ egg yolk cholesterol concentration 6 to 7\% (Lirette et al., 1993).

Nutrient Density Reducing the nutrient density of the diet was found to reduce yolk cholesterol. This effect could also be due to the increased fibre content of the low nutrient density diets, or to a reduction in weight gain by the hen, hence a reduction in lipogenesis. Varying the dietary levels of choline and/or methionine, which influence the lipid transport systems within the hen, did not produce a significant change in the egg cholesterol level (McDonald and Shafey, 1989).

Vitamins It has been suggested that several vitamins, such as vitamin A, niacin and ascorbic acid, reduce plasma cholesterol in the hen when added above normal levels (Hargis, 1988), however corresponding reductions in yolk cholesterol were not demonstrated. In fact, ascorbic acid reduced plasma cholesterol and raised the yolk level (McDonald and Shafey, 1989).

The zinc to copper ratio in the diet is thought to be a possible factor in determining the plasma level of cholesterol (Klevay, 1973), however, hens fed normal and low energy practical diets containing varied ratios of zinc and copper showed no 
significant differences in levels of either plasma or egg cholesterol (Helwig et al., 1978).

Supplementing the diets of white leghorn layers with $100 \mathrm{mg}$ and $150 \mathrm{mg}$ probiotic per $\mathrm{kg}$ of food reduced egg yolk cholesterol concentrations from $14.69 \mathrm{mg}$ to 11.28 and $11.37 \mathrm{mg} / \mathrm{g}$, respectively. Serum cholesterol values were similar between controls and treated birds when diets were supplemented with $100 \mathrm{mg} / \mathrm{g}$ probiotic but decreased from $176.5 \mathrm{mg} / \mathrm{dL}$ to $114.3 \mathrm{mg} / \mathrm{dL}$ when $150 \mathrm{mg} / \mathrm{g}$ probiotic was added to the diet (Mohan et al., 1995).

Bacteria A selected strain of Lactobacillus acidophilus when fed to laying hens at 4 million viable cells/g of feed reduced the cholesterol values in yolk by as much as $18.8 \%$ compared to control flocks. This was thought to be a possible result of a dramatic drop in serum cholesterol of $55 \%$ after the bacillus was added to the feed (Haddadin et al., 1996).

In summary, attempts to consistently reduce egg cholesterol levels by either altering the laying hen's diet, or through selection programs have only been marginally successful. The relative resistance of egg composition to alterations in diet apparently reflects the nutritional and structural requirements for avian embryonic development (Kuksis, 1992). Although dietary intake has been shown to influence egg cholesterol levels (Sutton, 1984, Sim et al., 1980), Beyer and Jensen (1989a) found that the cholesterol level of market eggs remained the same irrespective of the feed source 
There have been many attempts to reduce the cholesterol content of eggs but most have demonstrated that the cholesterol level is difficult to alter in the domestic fowl. Another approach to lowering egg cholesterol is the administration of pharmacological agents to laying hens.

\subsubsection{Pharmaceuticals}

During the past 25 years, many drugs that have been employed as hypercholesterolaemic agents in humans have been orally administered to chickens (Naber, 1983; Hargis, 1988). These drugs alter cholesterol metabolism, by interfering with either cholesterol synthesis or sterol excretion (Naber, 1983).

Examples of hypercholesterolaemic agents used are ethyl p-chlorophenoxyisobutyrate (CPIB) and D-thyroxine (Weiss et al., 1967b). Both CPIB and D-thyroxine injected subcutaneously reduced blood cholesterol but increased egg cholesterol concentration, with higher dosages having an adverse affect on egg production (Weiss et al., 1967a,b). Oral administration of Dthyroxine also increased the yolk cholesterol content, but did not produce the same decrease in plasma cholesterol. Secondary effects of D-thyroxine also included decreased yolk size and moulting (Weiss et al., 1967a,b).

Others noted that $80 \%$ of the cholesterol in the yolk was replaced with desmosterol after feeding Triparanol, (Naber et al., 1982; Burgess et al., 1962), and that Probucol reduced yolk cholesterol by 4 to $7 \%$ when fed at a level of $0.1 \%$ of the diet (Naber et al., 1982). 
Recent work by Elkin and Roger (1990), with the human hypercholesterolaemic drug lovastatin, suggested that HMG-CoA reductase inhibitors hold promise as effective egg cholesterol lowering agents. When PD 123244-15 [( \pm$)-\left(R^{*}, R^{*}\right)-3,4-$ dibromo- $\beta, \delta$-dihydroxy-2-(4-fluorophenyl)-5-(1-methylethyl)-1H-pyrrol-1heptanoic acid, sodium salt], a totally synthetic HMG-CoA reductase inhibitor, was fed to laying hens the egg cholesterol content varied inversely with the amount of dietary PD $123244-15$, with a maximum reduction of approximately $30 \%$ obtained at the highest drug level (Elkin et al., 1993). No drug residues were detected in either egg yolk or albumin extracts. Nevertheless, even if the Food and Drug Administration were to approve the procedure, Lovastatin is too expensive to use in commercial egg production (Crawford, 1990).

Little effect was found on yolk cholesterol levels even after inhibiting $43 \%$ of the activity of HMG-CoA reductase using 7-ketocholesterol (Vargus et al, 1986). The biological half life of plasma cholesterol in the hen is $36 \mathrm{~h}$, compared to $60 \mathrm{~h}$ in rats (Andrews et al., 1968). This indicates a far more rapid metabolism in the former species. Thus, the hen has a capacity to synthesise considerably more cholesterol than it normally produces.

Research into the effects of cholesterol-lowering using drugs fed orally or injected have often resulted in cessation of egg production or an accumulation of undesirable metabolites in the egg (Elkin et al., 1993). With current technology it appears unlikely that dietary changes, drugs or selective breeding will produce changes to egg cholesterol levels of significance to the human diet (Naber, 1983). 


\subsubsection{Removal of Egg Yolk}

Egg marketing authorities of Victoria and Queensland have developed and marketed "Scramblers" and "Yolk Free", respectively, low cholesterol foods containing largely egg albumin and vegetable oil. Egg yolk is partially replaced with vegetable oil or water then combined with egg white (Anon., 1991; Cully and Vollbrecht, 1992).

Trained flavour profile scores for scrambled eggs were shown to decrease as the level of egg white was increased. Although egg white contributes greatly to scrambled egg flavour by producing the very low threshold sulphur compounds, the yolk influences the scrambled egg flavour through the sweet note, and may be involved in retention of other flavour components (Warren and Ball, 1990).

Thus, many recombined egg products may fail to retain the desirable egg flavour demanded by consumers.

\subsubsection{Removal of Cholesterol- Containing Fat Components}

Other methods used to reduce cholesterol involve removing the cholesterolcontaining fat components. These methods have been effective in skim or low-fat milk. Unfortunately, the low-fat product may be organoleptically different from the full-fat product. Some food products produced may also depend upon the fat to provide function and bulk (Hegenbart, 1989). Manufacturers are then faced with the problem of having to replace not only the bulk, but also the flavour and functional qualities of the missing fat. 
A need has now been created to develop methods for reducing the cholesterol content in egg products without lowering organoleptic and functional properties.

Novel Food Ingredients in the Reformulation of Food Products

Intensive research and development efforts have been directed towards the development of novel food ingredients to be employed in the reformulation of food products (Gross, 1991).

Fat in cholesterol-reduced butter has been replaced with water and dairy proteins to literally dilute the cholesterol content. One disadvantage of this product is that it cannot be used for pan frying because it is likely to splatter. Some cheese manufacturers are also using milk proteins to replace cholesterol-containing butterfat in cheese products.

Emulsifiers have been used to replace the functional properties of fat, but not the bulk. These help to bind a product together and maintain the lubricity of mouthfeel that is normally lost when fat levels are reduced. In the baking industry cholesterol -containing fat is replaced on a 1:1 basis with a no-cholesterol fat. Manufacturers of frozen novelties, are replacing tropical oils in ice-cream products with soybean oil, for example, which does not increase cholesterol levels. However, these fatreplacers are awaiting Food and Drug Administration (FDA) approval, which may take several years (Hegenbart, 1989).

For traditional egg-based products, the lack of normal egg flavour and the poor flavour performance of egg substitutes, may be cause for complaints by consumers 
(Warren and Ball, 1990). Many research teams are seeking to find a way to remove cholesterol directly from the fats that contain it, thus eliminating many of the concerns involved with developing organoleptically acceptable products (Hegenbart, 1989).

\subsubsection{Extraction Processes}

\section{Steam Distillation}

Steam stripping used to deodorise fish oils also removes cholesterol from fish oil and other fats. Up to $93 \%$ of the cholesterol is removed while yielding up to $95 \%$ recovery of fat. In the process of steam stripping oil, temperatures as high as 220 $290^{\circ} \mathrm{C}$ are used to vaporise the oil into a chamber (Hegenbart, 1989). It is most unlikely that whole egg pulp or egg yolk could withstand such a treatment without suffering organoleptic deterioration. Both steam-stripping of cholesterol and fatreplacing techniques would also add cost to the final product.

\section{Organic Solvents}

Extraction of egg cholesterol using organic solvents has met with limited success. A large amount of cholesterol remains in the extracted egg, and the functional properties of the egg yolk are affected (Lebovics et al., 1992). Organic solvents remove phospholipids and denature proteins, both of which are responsible for functional properties (Froning et al., 1990). 
Using hexane and isopropanol as extraction solvents, Larsen and Froning (1981) fractionated egg yolk into an egg oil, a protein isolate and an aqueous component. It was suggested that the egg oil fraction could be used as a cooking oil and in pharmaceutical products and cosmetics, and the protein isolate as a food binder and emulsifier.

The egg oil fraction contained the highest concentration of cholesterol, which was removed (up to $40 \%$ ) by degumming, refining and bleaching. Analysis of the crude refined egg oil also showed a decrease in the ratio of unsaturated to saturated fatty acids as compared to intact egg yolk (Larsen and Froning, 1981).

Emulsion stability tests of the fractions revealed that neither was stable. Hexane greatly affected the emulsion stability by altering the protein integrity. When used to make mayonnaise, the viscosity associated with these emulsions were not as high as those found with mayonnaise using native egg yolk (Larsen and Froning, 1981). Also, in a sensory evaluation, panelists detected significantly more solvent odour in egg oil samples compared to corn and soybean oil. Egg oil also had high levels of rancidity (Larsen and Froning, 1981).

The development of egg oils has been described previously in literature (Romanoff and Romanoff, 1949; Anon., 1973). However, the production of these oils requires diethyl ether or chloroform extraction, which is unlikely to be acceptable for food products (Larsen and Froning, 1981). 


\section{Supercritical Fluid Extraction}

Capitalising on the unique properties of solvents above their critical conditions to extract target compounds from mixtures is the basic feature of a relatively new extraction and purification process known as supercritical fluid extraction (SFE).

An SFE system is made up of four basic components: a compressor, an extraction vessel, a temperature and pressure control unit and a separation vessel. An extraction solvent can be any liquid in a supercritical state. In such a system, a mixture is placed in contact with a supercritical extraction fluid in the extraction vessel. After the extraction, the supercritical fluid containing the extractives is introduced into the separation vessel and separated into product and solvent by reducing pressure. The solvent can be compressed and re-used. The system can be operated under different conditions to extract the target products.

Although there is some concern over the economics of SFE (Hegenbart, 1989), in spite of the high capital investment required, the process already has several commercial applications in the food industry.

Supercritical carbon dioxide is currently being used to extract flavours and decaffeinated coffee (Hegenbart, 1989). Food scientists are also investigating such applications as the extraction of spices, removal of oil from snack foods, extraction of oil from cotton seed, corn and soybeans, and extraction of oil from full-fat soy (Froning et al., 1990). Smaller scale successes have been achieved with fluid milk and butter (Hegenbart, 1989). Supercritical carbon dioxide has also been used to extract carotene and lutein from leaf protein concentrates (Favati 
et al., 1988). In other products, cheese for example, SFE may not be feasible as it could alter the flavour, texture and colour of the product (Best, 1989).

In eggs, up to $80 \%$ of egg cholesterol can be removed using carbon-dioxide as the extraction fluid. However, an economical technique to recover the cholesterol from the significant amount of co-extracted egg lipid is required as a prerequisite for commercial feasibility (Froning et al., 1990; Hung and Unger, 1993).

Froning et al. (1990) managed to extract two-thirds of the cholesterol from dried egg yolk at $306 \mathrm{~atm} / 45^{\circ} \mathrm{C}$ or $374 \mathrm{~atm} / 55^{\circ} \mathrm{C}$ compared to $163 \mathrm{~atm} / 40^{\circ} \mathrm{C}$ or $238 \mathrm{~atm} / 45^{\circ} \mathrm{C}$. The authors explained that this was due to the increased fluid density and greater volatility of cholesterol at the higher temperature/pressure conditions.

Phospholipids and protein were concentrated at the highest supercritical extraction conditions $\left(374 \mathrm{~atm} / 55^{\circ} \mathrm{C}\right)$ used, due to the significant reduction of total lipids (36\%) under these conditions. This was a desirable effect as the phospholipids are necessary for maintenance of good functional properties.

When used in sponge cake batter, SFE extracted egg yolk powder significantly improved sponge cake volume. This was observed at all extraction pressures and temperatures studied by Froning et al. (1990) except at $374 \mathrm{~atm} / 55^{\circ} \mathrm{C}$, ie. $163 \mathrm{~atm} / 40^{\circ} \mathrm{C} ; 238 \mathrm{~atm} / 45^{\circ} \mathrm{C}$ and $306 \mathrm{~atm} / 45^{\circ} \mathrm{C}$. The emulsion stability of mayonnaise, prepared with SFE extracted egg yolk powder was adversely affected by the $374 \mathrm{~atm} / 55^{\circ} \mathrm{C}$ extract, however the performance of yolk extracted at the milder treatment of $306 \mathrm{~atm} / 5^{\circ} \mathrm{C}$ was not significantly different from the control, 
generally indicating that supercritical extraction of cholesterol can be utilised without substantially impairing the stability of mayonnaise (Froning et al., 1990).

Extraction at increased temperatures and densities produced a dried egg yolk of lighter colour with less redness and yellowness indicating removal of some of the xanthophyll pigments (Froning et al., 1990).

Another undesirable effect of SFE is the increasing saturated to unsaturated fat ratio (S/U), as the temperature and/or pressure is increased (Froning et al., 1990). This was considered by Froning et al. (1990) to be a possible reflection of the concentration of the phospholipids in the extracted egg yolk powder rather than any specific fatty acid selectivity, since the extraction conditions used removed the majority of triglycerides in the system.

This increase in S/U fat may therefore, be of concern to health-conscious consumers who may be increasing their background diet of saturated fats even when consuming the low-cholesterol egg product (Froning et al., 1990).

Nonetheless, SFE extraction technology has excellent potential for production of a lower cholesterol, lower fat egg product.

\section{Cyclodextrin}

A natural, non-toxic compound derived from starch, $\beta$-cyclodextrin is composed of a ring of seven glucose units linked together by $\alpha-1,4$ glycosidic bonds (Bender, 1986). Cyclodextrin molecules can bind other compounds in their 
cavity, thereby stabilising, solubilising, or precipitating the compounds (Pszczola, 1988). When added to egg pulp, $\beta$-cyclodextrin forms an insoluble complex with cholesterol and cholesteryl ester.

The potential of cyclodextrin as an effective cholesterol removal reagent, was discovered by chance by Sidhu and Oakenfall, when investigating the absorption inhibiting effect of saponins on cholesterol in the gut for lowering plasma cholesterol (Davidson, 1990).

Cyclodextrin, unlike other proposed cholesterol-reduction technologies, is a cheap alternative, not requiring expensive and specialised equipment, and because it can be used at low temperatures, minimises the risk of microbiological spoilage or loss of flavour volatiles. Removal of up to $90 \%$ egg cholesterol has been described using this process (Cully and Vollbrecht, 1991).

$\beta$-cyclodextrin treated egg yolk was found to produce a low cholesterol egg product with similar compositional and functional properties similar to untreated egg yolk. The cholesterol reduced egg yolk contained less lipid and protein and more carbohydrate and ash than control egg yolk. Free and esterified cholesterol was reduced by $91.6 \%$ and $94.4 \%$, respectively (Awad et al. 1997).

Unfortunately, being a somewhat cumbersome batch process it inevitably leaves residues of the complexing agent in the product (Davidson, 1990). Even with an additional enzymatic treatment it is still difficult to completely remove all residues of $\beta$-cyclodextrin from the yolk (Cully and Vollbrecht, 1991). 
Though established as an acceptable food additive in a number of European countries and in Japan, $\beta$-cyclodextrin has yet to gain approval as a food additive by authorities in some countries, including the United States and Australia (Cully and Vollbrecht, 1991). This may also prove to be a major obstacle to the widespread use of the agent.

Various attempts have been made to solve the problem of $\beta$-cyclodextrin residue. For example, it has been suggested by Sidhu and Oakenfall that the molecule be chemically bonded to a support, such as a mass of silica beads; the adsorbent then used to extract cholesterol from milk or egg, and the beads washed for re-use (Davidson, 1990). However, more research is needed to optimise the process and to develop an economically viable and practical route for the production of lowcholesterol products (Davidson, 1990).

Consequently, cholesterol reduced egg products continue to be prepared by rather complex processes. There is a need for a simple technique to produce cholesterol free or cholesterol reduced egg yolk, without the loss of egg flavour, as a food ingredient for a variety of egg-based products.

\subsubsection{Enzymatic Degradation}

Industrial Significance of Enzymes

The food industry is the largest user of enzymes. Kindel (1981) has reported more than 2,500 enzymes being officially recognised by the International Union of Biochemistry, speculating that 25,000 natural enzymes exist (Neidleman, 1991). 
The world-wide market for industrial enzymes had an estimated value of $\$ 625$ million in 1990, with the U.S. as the largest sector; approximately $62 \%$ of the enzymes produced were destined for food applications (Leblond, 1990).

Enzymes can be used in the production of value-added ingredients and speciality chemicals using a variety of biocatalytic reactions, however the commercial development of such processes is often limited by the difficulties associated with the development of multi-enzyme reactions needed to achieve the desired final product (Penet, 1991).

In the food industry the largest use of enzymes is in starch processing followed by cheese production, fruit and vegetable juice, and barley and brewing (Leblond, 1990). Enzymes have also been used to create functional properties from deamidated soy protein (Hamada and Marshall, 1989), in the transesterification of unsaturated lipids by lipases (Osterberg et al., 1989), and for enhancing the functional properties of casein with trypsin (Chobert et al., 1988).

Today plants, animals and microbes are all commercial sources of useful industrial enzymes. Enzymes such as $\alpha$ - and $\beta$-amylases, proteases, amyloglucosidase, pentosanase, glucanase, and phytase used in bakery processing come from fungi and bacteria; the fungal genera Aspergillus, Rhizopus and Trichoderma are the chief sources of enzymes used in fruit and juice processing; chymosin, used by the dairy industry to coagulate milk, is obtained by saline extraction of the abomasum, or fourth stomach, from unweaned calves; the fungi, Mucor miehei, Mucor pusillus and Endothia parasitica are sources of chymosin-like proteases used as commercial substitutes for calf rennet in the manufacture of cheese; and 
commercially available lactase preparations are commonly purified from the yeast Kluyveromyces lactis (formerly Saccharomyces lactis) and the fungi Aspergillus oryzae (Dziezak, 1991).

Other examples which illustrate the potential of enzymes as catalysts in the production of food ingredients are the use of a lipase in the regio-specific transformation of fats and oils to produce $\beta$-monoglycerides and the use of cholesterol reductase to convert cholesterol into a non-toxic compound (Gross, 1991).

\section{Immobilisation of Enzymes}

The stability of enzymes is important for the economy of their industrial applications since highly purified enzyme preparations are expensive.

Storage stability depends upon the physical form of enzyme preparations and solid forms generally have higher stability than liquid forms (Randolph $e t$ al., 1988). Immobilisation is a means of creating a solid enzyme form and essentially eliminates the danger of proteolysis, however the risk of microbial degradation is higher. Immobilisation of an enzyme onto a solid support allows processors to take advantage of the enhanced reusability of this enzyme form (Dziezak, 1991). The continuous process of lactose hydrolysis relies on lactase that has been immobilised on a solid support such as a column-type reactor (Dziezak, 1991). 


\subsubsection{Microbiological Degradation}

Work done by Watanabe et al. (1986) suggested that cholesterol decomposing microorganisms in nature existed not only among Mycobacterium and Nocardia species as had been previously recognised but were also widely distributed among actinomycetes and bacteria.

Cholesterol-degrading bacteria (16 strains) have been isolated from foods of animal origin such as butter, bacon, pork fat and chicken fat. Most have been identified as Rhodococcus equi or Rhodococcus erythropolis (Ferriera and Tracey, 1984; Watanabe et al., 1986). These cholesterol-degrading bacteria are known to use cholesterol as the sole source of carbon for growth and have been found to have similar cholesterol-degrading ability but with some differences in the production of cholesterol oxidase (COD) (Aihara et al., 1986; Watanabe et al., 1986).

Microbiological degradation of cholesterol by some strains of Nocardia, Brevibacterium, Corynebacterium or Streptomyces have also been reported (Aihara et al., 1986, 1988a,b). Some strains of these organisms accumulate steroid intermediates, with consequent adverse health effects, however other strains which can degrade cholesterol without accumulating steroid intermediates have also been isolated (Aihara et al., 1988b).

Isolated intracellular and extracellular COD of various strains of cholesteroldegrading bacteria degraded over $70 \%$ of the cholesterol in a pure cholesterol preparation within 3 days (Watanabe et al., 1986). Johnson and Somkuti (1990) 
also found Rhodococcus strains could reduce up to $80 \%$ of cholesterol in a pure cholesterol preparation after 7 days growth. Although these findings indicate that enzymatic treatment could be used for egg cholesterol degradation, for any commercial application it would be necessary to shorten the degradation time of 3 days reported in these studies.

\subsection{Project Aims and Objectives}

The overall objective of this project is to define the parameters affecting the production of low cholesterol egg yolk products by enzymatic means.

This will include the following specific aims:

- the development of a rapid and simple method for the assay of cholesterol in egg yolk and other egg-based food products,

- the investigation of the cholesterol-degrading ability of cholesterol reductase and COD in egg yolk and

- the cholesterol-degrading activities of $R$. equi, R. erythropolis and two other unspeciated isolates of Rhodococcus . 
CHAPTER 2

Materials and Methods 


\subsection{Cholesterol Assay Conditions}

\section{Solvents}

- Absolute ethanol, analytical grade, Sigma Chemical Co.

- Chloroform, HPLC grade, Ajax Chemicals.

- Diethyl ether, laboratory reagent, Ajax Chemicals.

- Hexane, laboratory reagent, Ajax Chemicals.

- Methanol, Absolute, Sigma Chemical Co.

- Petroleum ether, AnalaR, BDH Chemicals, Australia Pty Ltd.

\section{Chemicals}

- di-potassium hydrogen orthophosphate, analytical reagent, BDH Chemicals.

- di-Sodium hydrogen orthophosphate, analytical reagent, BDH Chemicals.

- $\quad \mathrm{N}$-methyl-N-(trimethylsilyl)-trifluoroacetamide $\mathrm{C}_{6} \mathrm{H}_{12} \mathrm{~F}_{3} \mathrm{NOSi}$ (MSTFA), Sigma Chemical Co.

- $\quad \mathrm{N}$-O-bis-(trimethylsilyl)-trifluoroacetamide $\mathrm{C}_{8} \mathrm{H}_{18} \mathrm{~F}_{3} \mathrm{NOSi}_{2}$ (BSTFA), Alltech (Aust) Pty. Ltd.

- Potassium dihydrogen orthophosphate, analytical reagent, BDH Chemicals.

- Potassium hydroxide, analytical reagent, BDH Chemicals.

- Sodium dihydrogen orthophosphate, analytical reagent, Ajax Chemicals. 
Chromatography Standards

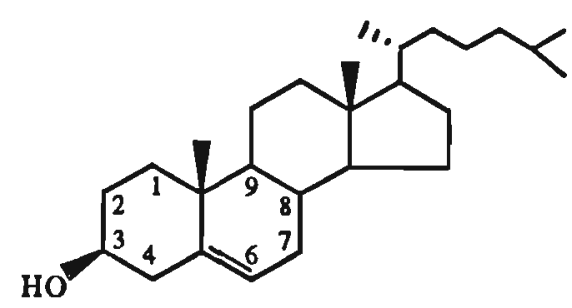

- Cholesterol, (Cholest-5-en-3ß-ol) $\mathrm{C}_{27} \mathrm{H}_{46} \mathrm{O}$, mol wt 386.64g/mol, 99 $\%$, Sigma Chemical Co.

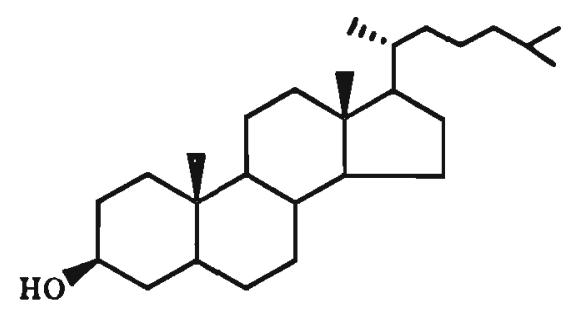

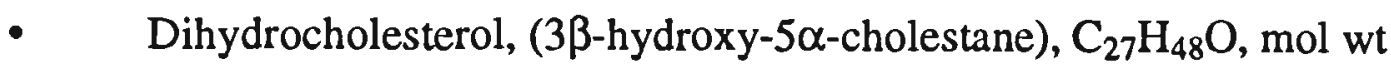
$388.65 \mathrm{~g} / \mathrm{mol}$, approx. 95\%, Sigma Chemical Co.

- Octacosan-1-ol $\left(\mathrm{C}_{28} \mathrm{H}_{58} \mathrm{O}\right)$, mol wt $410.74 \mathrm{~g} / \mathrm{mol}$, approx. $99 \%$ by capillary GC, Sigma Chemical Co.

\section{Instruments}

Hewlett Packard 5890 Gas Chromatograph

Column :

Phase thickness:

Injector mode:

Injection volume:

Detector:
BP- $10,25 \mathrm{~m} \times 0.25 \mathrm{~mm}$ i.d.

$0.25 \mu \mathrm{m}$

split ratio 30:1

$1 \mu \mathrm{L}$ in chloroform

FID or MSD Hewlett Packard 5971A

Mass Selective Detector 


$\begin{array}{lll}\text { Temperatures: } & \text { Injector } & 250^{\circ} \mathrm{C} \\ & \text { Detector } & 320^{\circ} \mathrm{C} \\ & \text { Oven } & 285^{\circ} \mathrm{C} \text { isothermal } \\ & 0.8 \mathrm{~mL} / \mathrm{min} \mathrm{He} \\ \text { Carrier gas: } & \text { scan } \\ \text { Acquisition mode: } & 1.1 / \mathrm{s} \\ \text { Scan rate: } & 40-550 \mathrm{amu} \\ \text { Scan range: } & 12 \mathrm{~min} \\ \text { Run time: } & \text { Hewlett Packard Vectra 386/25 } \\ \text { Work station: } & \text { HP ChemStation }\end{array}$

\section{Varian 3700 Gas Chromatograph}

Column :

Phase thickness:

Injector mode:

Injection volume:

Detector:

Temperatures:

Carrier gas:

Run time:

Range:

Integrator:

Chart speed:
BP- $10,25 \mathrm{~m} \times 0.25 \mathrm{~mm}$ i.d.

$0.25 \mu \mathrm{m}$

split ratio 50:1

$0.5 \mu \mathrm{L}$ in chloroform

FID

$\begin{array}{ll}\text { Injector } & 300^{\circ} \mathrm{C} \\ \text { Detector } & 350^{\circ} \mathrm{C} \\ \text { Oven } & 285^{\circ} \mathrm{C} \text { isothermal }\end{array}$

$0.8 \mathrm{~mL} / \mathrm{min} \mathrm{He}$

$10 \mathrm{~min}$

$10^{-11} \mathrm{~A} / \mathrm{mV}$

Shimadzu CR-1B Chromatopac

$0.5 \mathrm{~cm} / \mathrm{min}$ 


\subsubsection{Cholesterol Determination}

Cholesterol was determined using a modification of the method described by Kovacs (1990).

A $0.1 \mathrm{~g}$ sample of egg yolk, either weighed directly or dispensed as a $0.5 \mathrm{~mL}$ aliquot of a $0.2 \mathrm{~g} / \mathrm{mL}$ dispersion in $0.1 \mathrm{M}$ phosphate buffer $(\mathrm{pH})$, was placed into a $15 \mathrm{mM}$ x $100 \mathrm{mM}$ test-tube fitted with a teflon-lined screw cap. $1 \mathrm{M} \mathrm{KOH}$ in absolute ethanol $(2 \mathrm{~mL})$, containing $0.5 \mathrm{mg} / \mathrm{mL}$ of internal standard, dihydrocholesterol, was added and the sealed tube heated at $100^{\circ} \mathrm{C}$ for $1 \mathrm{~h}$. After the digestion, diethyl ether $(3 \mathrm{~mL})$ and water $(2 \mathrm{~mL})$ were added, the mixture was vortexed and the ether phase taken into a second test-tube fitted with a teflon-lined screw cap. The aqueous phase in the first test-tube was extracted with a further $2 \mathrm{~mL}$ diethyl ether. A few drops of saturated $\mathrm{NaCl}$ solution was required to avoid emulsion formation. The ether phase was combined, washed three times with water $(1 \mathrm{~mL})$ and then passed through a $4 \mathrm{~mm} \times 25 \mathrm{~mm}$ column of anhydrous $\mathrm{Na}_{2} \mathrm{SO}_{4}$, to remove moisture, into a $1.5 \mathrm{~mL}$ glass reaction vial. The ether was evaporated in a stream of $\mathrm{N}_{2}$ at $40^{\circ} \mathrm{C}$, $100 \mu \mathrm{L}$ MSTFA added and the sealed vial heated at $100^{\circ} \mathrm{C}$ for $15 \mathrm{~min}$. The reaction product was diluted with $500 \mu \mathrm{L} \mathrm{CHCl}_{3}$ and $1 \mu \mathrm{L}$ taken for gas chromatography using the Hewlett Packard gas chromatograph and the conditions outlined in Chapter 2.1. Under these chromatographic conditions, retention times of the TMS derivatives of cholesterol and dihydrocholesterol were typically 10 and 10.3min respectively.

The identification of cholesterol and dihydrocholesterol in gas chromatography traces was facilitated by comparison of retention times with those of known standards chromatographed under identical conditions. Quantification of 
cholesterol was achieved by comparison of the areas of the cholesterol and dihydrocholesterol peaks and the application of a small factor to recognise a slight difference in the response of each of these compounds in the flame ionisation detector.

\subsubsection{Cholesterol Content in Multi-component Foods}

A survey of cholesterol content of several typical western foods and their oriental analogues, available in Melbourne markets, was conducted using the method described in section 2.1.1. with the FID fitted to the gas chromatograph. Fresh noodles were milled in a Bamix ${ }^{\circledR}$ miller, dried overnight at $100^{\circ} \mathrm{C}$, and milled again. Other foodstuffs were also milled in a Bamix ${ }^{\circledR}$ miller, except pork cake which was mixed with a glass stirring rod.

A total of 15 Western foods and 10 Asian foods were assayed for cholesterol, and data was expressed as average mg cholesterol/g samplets.e $(n=3)$.

\subsection{Optimisation of Cholesterol Assay Conditions}

\subsubsection{Internal Standards}

In order to compare the effectiveness of the two internal standards used in this work, a $0.1 \mathrm{~g}$ sample of fresh egg yolk was assayed for cholesterol using either dihydrocholesterol or octacosan-1-ol. 


\subsubsection{Substrate}

A $0.2 \mathrm{~g} / \mathrm{mL}$ suspension of egg yolk was prepared in $0.1 \mathrm{M} \mathrm{pH} 7$ phosphate buffer, and $0.5 \mathrm{~mL}$ aliquots assayed for cholesterol $(\mathrm{n}=4)$. The same egg yolk was assayed fresh $(0.1 \mathrm{~g})(\mathrm{n}=4)$.

\subsubsection{Silylation}

A number of silylating agents are available for the derivatisation of the hydroxyl groups of steroids. The two reagents studied for the formation of trimethylsilyl (TMS) ether derivatives of cholesterol and the internal standards were N-O-bis(trimethylsilyl)-trifluoroacetamide (BSTFA) and N-methyl-N-(trimethylsilyl)trifluoroacetamide (MSTFA) (Figures 2.1 and 2.2). The silylated structures for cholesterol and internal standard dihydrocholesterol are shown in Figures 2.3 and 2.4, respectively. The formula for silylated octacosan-1-ol is $\mathrm{C}_{28} \mathrm{H}_{57} \mathrm{OSi}\left(\mathrm{CH}_{3}\right)_{3}$.<smiles>C[Si](C)(C)N[C@@H](O[Si](C)(C)C)C(F)(F)F</smiles>

Figure 2.1 The structure of BSTFA (N-O-bis-(trimethylsilyl)-trifluoroacetamide), $\mathrm{C}_{8} \mathrm{H}_{18} \mathrm{~F}_{3} \mathrm{NOSi}_{2}$, Alltech (Aust) Pty. Ltd.

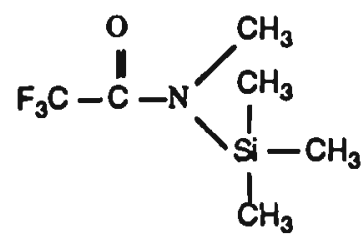

Figure 2.2 The structure of MSTFA (N-methyl-N-(trimethylsilyl)-trifluoroacetamide), $\mathrm{C}_{6} \mathrm{H}_{12} \mathrm{~F}_{3} \mathrm{NOSi}$, Sigma Chemical Co. and Alltech (Aust) Pty. Ltd. 


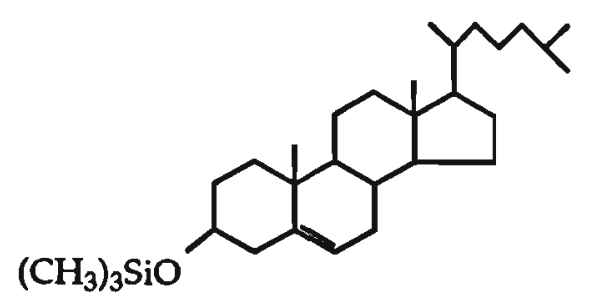

Figure 2.3 The structure of MSTFA silylated cholesterol, $\mathrm{C}_{27} \mathrm{H}_{45} \mathrm{OSi}\left(\mathrm{CH}_{3}\right)_{3}$.

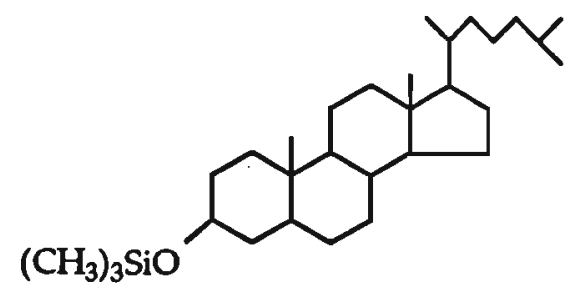

Figure 2.4 The structure of MSTFA silylated dihydrocholesterol, $\mathrm{C}_{27} \mathrm{H}_{47} \mathrm{OSi}\left(\mathrm{CH}_{3}\right)_{3}$.

\section{Silylation Reaction Time Studies}

A study of the silylation reaction was conducted in order to establish conditions necessary for complete derivatisation. A mixture containing equal amounts of cholesterol and octacosan-1-ol $(1 \mathrm{mg} / \mathrm{mL})$ in chloroform was prepared. The mixture $(1 \mathrm{~mL})$ was dispensed into septum-stoppered glass vials, evaporated to dryness under a stream of nitrogen, and derivatised with silylating agent (either BSTFA or MSTFA) for varying time periods $(0,1,2,3,5,10,20,30,40,50$ and $60 \mathrm{~min}$ ) with intermittent shaking at room temperature. A four fold excess of silylating reagent $(100 \mu \mathrm{L})$ was routinely used for these reactions. Each sample was diluted with $100 \mu \mathrm{L}$ chloroform, and $0.5 \mu \mathrm{L}$ taken for gas chromatography using the equipment and conditions outlined in section 2.1. 


\subsubsection{Gas Chromatography}

\section{Position of Capillary Column in Injector of Varian Gas Chromatograph}

$1 \mathrm{~mL}$ aliquots of a mixture containing equal amounts of cholesterol and octacosan-1-ol $(1 \mathrm{mg} / \mathrm{mL})$ was prepared, and were evaporated to dryness in septum-stoppered glass vials and derivatised with excess MSTFA $(100 \mu \mathrm{L})$ in preparation for gas chromatography.

The capillary column was inserted $11 \mathrm{~cm}$ into the injector port liner of the Varian gas chromatograph and progressively lowered until $4 \mathrm{~cm}$ remained inserted. At each centimetre interval, a $0.5 \mu \mathrm{L}$ injection of the standard mixture of derivatised cholesterol and octacosan-1-ol in chloroform $(1 \mathrm{mg} / \mathrm{mL})$ was made. The peak area ratios and peak heights of the standards were recorded.

\section{Relative Response Factors}

One millilitre aliquots of a mixture containing equal amounts of cholesterol and each internal standard were evaporated to dryness at $40^{\circ} \mathrm{C}$ and then derivatised with excess MSTFA in preparation for gas chromatography.

The response factor for each of the internal standards was determined in relation to cholesterol by measuring the peak area of the internal standard relative to cholesterol. 


\subsubsection{Reproducibility and Recovery}

Reproducibility and recovery data were collected for both egg yolk and egg-based food products. The yolks of three eggs were combined and mixed until homogeneous and $0.1 \mathrm{~g}$ samples of the homogenate were taken for testing. Uncooked egg noodles, purchased locally, were ground to uniformity using a Bamix ${ }^{\circledR}$ mill and $0.1 \mathrm{~g}$ samples taken for testing.

For the comparison of cholesterol values obtained from the original and modified extraction procedure of Kovacs, replicate samples (eight and seven, respectively) were taken and carried through each procedure. The reproducibility was expressed as a percentage coefficient of variation and the mean cholesterol was expressed as mg cholesterol/g samplets.e.

The efficiency of recovering cholesterol by each extraction procedure was tested using replicate samples (eight and seven) to which a known amount of pure cholesterol had been added; cholesterol dissolved in ethanolic potassium hydroxide solution (1M) was added to each sample prior to saponification. The \% recovery was determined by subtracting the average peak area of cholesterol present in unspiked samples from that of the spiked samples.

\subsection{Enzymatic Treatment of Egg Yolk}

\section{Organic Material}

- $\quad$ Cucumber leaves ( 3 week old) 
Enzymes

- Cholesterol oxidase derived from Brevibacterium species (Bs), lyophilised powder, Sigma Chemical Co.

- Cholesterol oxidase derived from Nocardia erythropolis (Ne), lyophilised powder, Sigma Chemical Co.

- Cholesterol oxidase derived from Pseudomonas fluorescens (Pf), lyophilised powder, Sigma Chemical Co.

- $\quad$ Cholesterol oxidase derived from Streptomyces species (Ss), lyophilised powder, Sigma Chemical Co.

\section{Solvents}

- Absolute ethanol, analytical grade, Sigma Chemical Co.

- Acetonitrile, ACS reagent, Sigma Chemical Co.

- Chloroform, HPLC grade, Ajax Chemicals.

- Diethyl ether, laboratory reagent, Ajax Chemicals.

- Isopropanol anhydrous, Sigma Chemical Co.

- Methanol, Absolute, Sigma Chemical Co.

\section{Chemicals}

- Amberlite resin, XAD-4, Sigma Chemical Co.

- Ammonium nitrate, analytical reagent, Mallinckrodt Australia.

- Ascorbic acid, analytical reagent, BDH Chemicals. 
- $\quad 2$ ', 7'-dichlorofluorescein, spray reagent, $0.2 \%$ in ethanol, Sigma Chemical Co.

- Dithiothreitol, 99+\%, Sigma Chemical Co.

- Ethylenediaminetetraacetic acid, analytical reagent, Ajax Chemicals.

- $\quad$ Glycerol, 99+\%, Sigma Chemical Co.

- di-potassium hydrogen orthophosphate, analytical reagent, BDH Chemicals.

- di-sodium hydrogen orthophosphate, analytical reagent, $\mathrm{BDH}$ Chemicals.

- Ferrous sulphate heptahydrate, 'Baker analysed' reagent, J. T. Baker Chemical Co.

- Liquid nitrogen, Liquid Nitrogen Services Pty. Ltd.

- Magnesium sulphate, analytical reagent, BDH Chemicals.

- $\quad$ MSTFA (N-methyl-N-(trimethylsilyl)-trifluoroacetamide), Sigma Chemical Co.

- 2-(N-morpholino)ethanesulphonic acid, crystalline, 99+\%, Sigma Chemical Co.

- Nicotinamide adenine dinucleotide phosphate (NADPH)

- $\quad$ Polyvinylpolypyrrolidone (PVPP), Polyclar AT, Sigma Chemical Co.

- Potassium dihydrogen orthophosphate, analytical reagent, BDH Chemicals.

- Potassium hydroxide, analytical reagent, BDH Chemicals.

- Sodium dihydrogen orthophosphate, analytical reagent, Ajax Chemicals.

- $\quad$ Sodium metabisulphite, ACS reagent, Sigma Chemical Co.

- Sodium sulphate anhydrous, analytical reagent, Ajax Chemicals. 
- Sucrose, analytical reagent, Ajax Chemicals.

- $\quad$ Thimerosal, approx. 98\% (HPLC), Sigma Chemical Co.

Chromatography Standards

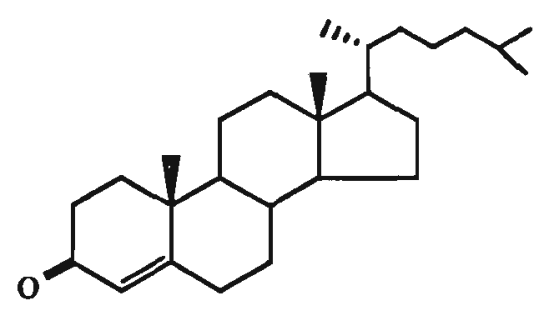

- Cholest-4-en-3-one (3-keto-4-cholestene), $\mathrm{C}_{27} \mathrm{H}_{44} \mathrm{O}$, mol wt 384.6g/mol, approx. 97\%, Sigma Chemical Co.

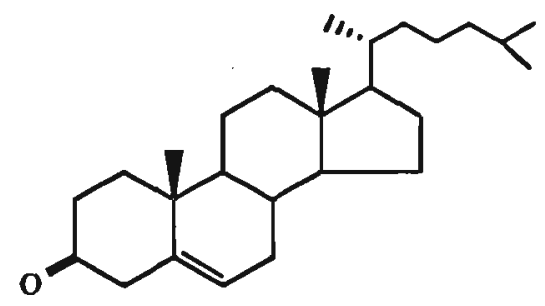

- Cholest-5-en-3-one (3-keto-5-cholestene), $\mathrm{C}_{27} \mathrm{H}_{44} \mathrm{O}$, mol wt 384.6g/mol, approx. 97\%, Sigma Chemical Co.

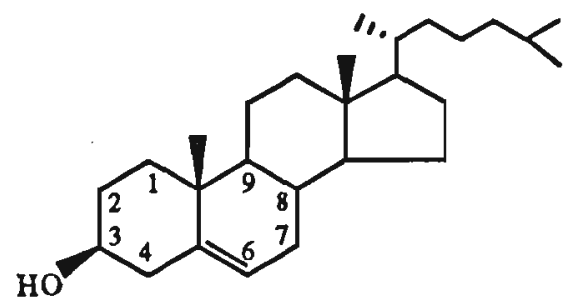

- $\quad$ Cholesterol (cholest-5-en-3ß-ol) $\mathrm{C}_{27} \mathrm{H}_{46} \mathrm{O}$, mol wt $386.64 \mathrm{~g} / \mathrm{mol}, 99 \%$, Sigma Chemical Co. 


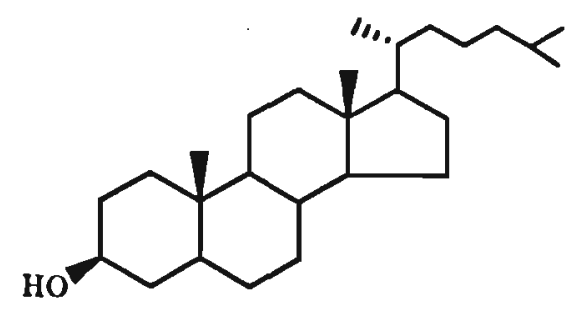

- Dihydrocholesterol ( $3 \beta$-hydroxy-5 $\alpha$-cholestane), $\mathrm{C}_{27} \mathrm{H}_{48} \mathrm{O}$, mol wt $388.65 \mathrm{~g} / \mathrm{mol}$, approx. $95 \%$, Sigma Chemical Co.<smiles>C[C@H](CCCC(C)(C)O)[C@H]1CCC2C3CC=C4CC(O)CC[C@]4(C)[C@H]3CC[C@]21C</smiles>

- 25-hydroxycholesterol (cholest-5-en-3 $\beta, 25$-diol), $\mathrm{C}_{27} \mathrm{H}_{46} \mathrm{O}_{2}$, mol wt 402.7g/mol, pfs, Sigma Chemical Co.<smiles>CC(C)CCC[C@H](C)[C@H]1CCC2C3C(=O)C=C4CC(O)CC[C@]4(C)C3CC[C@]21C</smiles>

- 7-ketocholesterol (cholest-5-en-3 $\beta$-ol-7-one), $\mathrm{C}_{27} \mathrm{H}_{44} \mathrm{O}_{2}$, mol wt 400.6g/mol, minimum 90\%, Sigma Chemical Co.

\section{Egg Sample}

Batches of liquid egg yolk were prepared from 61 locally purchased fresh Grade AAA average-sized eggs. Yolks were separated from albumin and blended at low speed in a domestic blender until homogeneous. A $0.2 \mathrm{~g} / \mathrm{ml}$ suspension of yolk in $0.1 \mathrm{M} \mathrm{pH7}$ potassium phosphate buffer containing $0.02 \%$ thimerosal as an antibacterial agent was prepared and $20 \mathrm{ml}$ portions stored at $-4^{\circ} \mathrm{C}$ until required. 


\subsubsection{Cholesterol Reductase}

\section{Method of Extraction from Cucumber Leaves}

A cholesterol reductase enriched solution that could be used to decrease the cholesterol concentration in egg yolk was prepared from cucumber leaves (Beitz et al., 1990).

The method involved grinding $20 \mathrm{~g}$ of young cucumber leaves ( 3 to 6 weeks old) in liquid nitrogen using a mortar and pestle sitting in an ice bath. Finely ground dry amberlite resin ( $1 \mathrm{~g}$ dry $\mathrm{w} / \mathrm{g}$ fresh cucumber leaves) was then mixed into the frozen powder and the mixture was held in the ice bath for several minutes to allow the enzymatic release of isothiocyanates.

Homogenisation buffer (sodium/potassium phosphate buffer, $100 \mathrm{mM}, \mathrm{pH} 6.5$ ) containing sucrose $(150 \mathrm{mM})$, sodium metabisulphite $(10 \mathrm{mM})$, ascorbic acid $(10 \mathrm{mM})$, dithiothreitol $(5 \mathrm{mM})$, and ethylenediaminetetraacetic acid $(0.1 \mathrm{mM})$, was slowly added to the mixture ( $5 \mathrm{~mL} / \mathrm{g}$ cucumber leaves) and proteins were extracted by stirring in insoluble PVPP (5g hydrated wt $g$ fresh cucumber leaves) to produce a thick slurry. Insoluble PVPP is used to remove those plant phenolic compounds which form strong hydrogen bonded complexes with proteins, however, some phenolic compounds, as well as certain other interfering secondary products, are not bound effectively by PVPP (Loomis et al., 1979).

The slurry was then squeezed through four layers of gauze into a separating funnel suspended over a glass column packed with amberlite XAD-4 resin. The tap of the 
funnel was opened and the filtrate allowed to pass down the column. The amberlite resin facilitated the removal, through hydrophobic interactions, of hydrophobic secondary products and surface active materials, including phenolics and contaminating monoterpenes from the plant enzyme extract (Loomis et al. 1979). This procedure was repeated a second time after regeneration of the column by washing with methanol, followed with water.

The filtrate was then centrifuged at $27,000 \mathrm{Xg}$ (approx. 18,000 rpm) for $20 \mathrm{~min}$ at $4^{\circ} \mathrm{C}$ and the supernatant passed down an amberlite XAD-4 resin column to remove any persistent monoterpene contaminants.

The supernatant was centrifuged once more, this time at 140,000Xg (approx. $41,000 \mathrm{rpm}$ ) for $1 \mathrm{~h}$ at $4^{\circ} \mathrm{C}$. The resulting supernatant (cytosol) was then dialysed against a solution of dialysis buffer: $10 \mathrm{mM}$ sodium/potassium phosphate buffer (pH6.5) containing $20 \mathrm{mM}$ 2-[N-morpholino]ethanesulphonic acid, $0.5 \mathrm{mM}$ dithiothreitol, and $10 \%(\mathrm{v} / \mathrm{v})$ glycerol, at $4^{\circ} \mathrm{C}$ for $24 \mathrm{~h}$, with the dialysis buffer being changed at least 3 times.

The clear dialysed concentrate was employed as the cholesterol reductase-enriched preparation.

\section{Purification and Preparation of XAD-4 Amberlite Resin}

Purification of the amberlite XAD-4 resin is important before use. A purification procedure followed by Loomis et al. (1979) was adopted whereby approximately 
$30 \mathrm{~g}$ of resin was sequentially extracted with methanol, acetonitrile and petroleum ether $(100 \mathrm{~mL})$ in a Soxhlet extractor for $8 \mathrm{~h}$ each.

The beads of amberlite resin were then combined and stored under methanol in a glass column. Prior to use the column was washed with water.

\section{Purification and Preparation of PVPP}

PVPP was purified by boiling for $10 \mathrm{~min}$ in $10 \%$ hydrochloric acid and washing with glass distilled water until free of chloride. The absence of chloride was measured by adding silver nitrate $\left(\mathrm{AgNO}_{3}\right)$ dropwise into the boiled mixture until no colour change was observed. The washing process was hastened by neutralising with potassium hydroxide after several changes of wash water. The purified PVPP was then dried for storage and when required hydrated in homogenisation buffer for several hours before use

\section{Cholesterol Degrading Capacity of Cholesterol Reductase Enriched Extract}

The cholesterol degrading capacity of the cholesterol reductase extract was investigated with both pure cholesterol and egg yolk. Cholesterol in isopropanol (1 $\mathrm{mg} / \mathrm{mL}$ ) and egg suspension containing $0.1 \mathrm{~g}$ yolk were separately incubated with $1 \mathrm{~mL}$ of cholesterol reductase enriched extract and $1 \mathrm{mM} \mathrm{NADH}$ for $0,75 \mathrm{~min}$ or $24 \mathrm{~h}$ at $37^{\circ} \mathrm{C}$, and residual cholesterol was determined.

One hundred and twenty test-tubes were randomly assigned treatments. Treatments were extract or no extract, incubation time of $0,75 \mathrm{~min}$ or $24 \mathrm{~h}$ and 
$0.5 \mathrm{~mL}$ yolk suspension $(0.2 \mathrm{~g} / \mathrm{mL})$ or $0.5 \mathrm{~mL}$ cholesterol in isopropanol $(1 \mathrm{mg} / \mathrm{mL})$. Treatments $(n=10)$ were incubated at $37^{\circ} \mathrm{C}$ and cholesterol was determined at the end of each incubation period. Test-tubes were assayed for cholesterol in numerical order. The arrangement of the test-tubes in the incubator was fully randomised.

The experiment was conducted as a completely randomised design (Snedecor et al., 1967), and data was analysed as a substrate by (enzyme by incubation time, plus control) factorial.

\subsubsection{Cholesterol Oxidase}

Cholesterol oxidases (COD) derived from Ss, Bs, Ne and Pf were purchased from Sigma Chemical Company, St. Louis, Mo as lyophilised powders. Cholesterol oxidase from Nocardia erythropolis was reconstituted in $0.1 \mathrm{M}$ pH5 potassium phosphate buffer and the others in the same type of buffer but at $\mathrm{pH} 7$. These pHs have been reported as being the most suitable at which to store these enzymes without appreciable loss of activity (Smith and Brooks, 1974).

Since the incubation $\mathrm{pH}$ may have a strong effect on the enzymatic activity, the degradation capacity of each enzyme was measured at $\mathrm{pH} 7$, a $\mathrm{pH}$ at or close to their optimum physiological pH based on available literature. Cheillan et al. (1989) observed the activity of Pf COD remaining constant between pH6.5 and 8.5. Uwajima et al. (1974) have shown that the enzyme isolated from Brevibacterium had a broad pH optimum in the range of pH6.0 to 8.5 and Noma and Nakayama 
(1976) observed a pH optimum of 7.0 for Ne and Bs COD, and $\mathrm{pH} 7.5$ for the enzyme from Streptomyces.

For COD activity, one enzyme unit (1U) is defined as the amount of cholesterol oxidase that will promote the conversion of $1 \mu$ mole of cholesterol to cholest-4-en3-one per minute (Figure 2.5) under the prescribed test conditions (Cheillan et al., 1989).

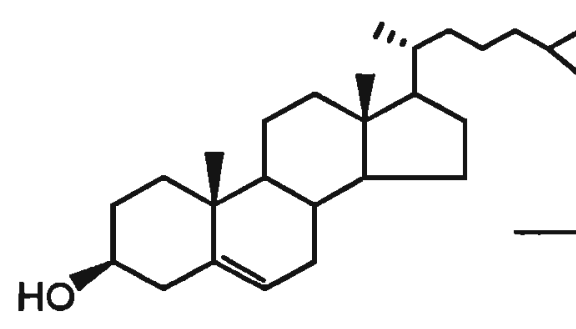

Cholesterol

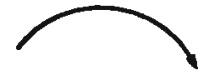

$\mathrm{H}_{2} \mathrm{O}_{2} \quad \mathrm{O}_{2}$

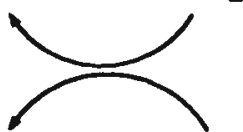

Enz-FAD Enz-FADH ${ }_{2}$

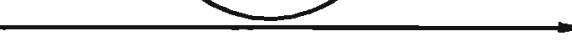

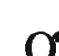

Cholest-4-en-3-one

Figure 2.5 The oxidation of cholesterol to cholestenone catalysed by cholesterol oxidase (Cheillan et al., 1989).

Cholesterol Degrading Capacity of Cholesterol Oxidase

\section{Effect of Time and Temperature}

The effect of incubation time on the cholesterol degrading capacity of each enzyme was initially investigated. Yolk suspensions containing $0.1 \mathrm{~g}$ yolk (approximately $3.9 \mu$ mole cholesterol) were added to tightly capped (teflon-lined) $10 \mathrm{~mL}$ test tubes and incubated, in triplicate, with $3.9 \mathrm{U}$ of each enzyme at 4,25 and $37^{\circ} \mathrm{C}$. Residual cholesterol was determined after $3,6,9,12,24,48$ and $72 \mathrm{~h}$ by gas chromatography. The experiment was not replicated. Results of this investigation 
were used to select $12 \mathrm{~h}$ as a suitable time for a more detailed study of incubation temperature.

To four of seven test-tubes containing $0.1 \mathrm{~g}$ yolk in buffer suspension, $3.9 \mathrm{U}$ of cholesterol oxidase enzyme from each of four types (Ne, Ss, Bs and Pf) was added. The other three test-tubes contained no enzyme and were treated as controls. These treatments were randomly positioned into test-tube racks, and the racks were randomly assigned to incubators at each of the temperatures, $5,15,25$, 37,45 and $60^{\circ} \mathrm{C}$. Incubation time was $12 \mathrm{~h}$.

The experiment was replicated on three different days and described as a split-plot type design (Snedecor et al., 1967). Days were considered as blocks, incubators as plots and test-tubes containing yolks as the sub-plots. Temperature was the main plot treatment and the enzymes (including controls) were the sub-plot treatments. The main temperature effect, and the temperature by enzyme type interaction, were divided into orthogonal polynomial components up to cubic order to determine if there were linear and quadratic trends.

The data was analysed as a temperature by enzyme type factorial with the control being considered in the analysis as an enzyme type. Pairs of means were compared using Fisher's least significant difference (LSD) at the 5\% level (Snedecor et al., 1967).

\section{Effect of Concentration}

Yolk suspensions containing $0.1 \mathrm{~g}$ yolk cholesterol were incubated with $0,0.5,1$, 2,4 and $8 \mathrm{U}$ enzyme/ $\mu$ mole egg cholesterol for $2 \mathrm{~h}$ at $45^{\circ} \mathrm{C}$ and residual cholesterol was determined. The analysis was replicated on three days, with each day 
considered as a block (Snedecor et al., 1967). Within each block there was one test-tube sample of each enzyme type by concentration combination plus two control samples (without enzyme). The arrangement of the test-tubes placed in the incubator was fully randomised. The data was analysed as an enzyme type by concentration factorial plus an external non-enzyme control treatment, with blocking for time. Within the factorial the concentration effects and concentration by enzyme type interaction were divided into orthogonal polynomial components up to cubic order to determine if there were linear and quadratic trends. Pairs of means were compared using Fisher's least significant difference (LSD) at the 5\% level (Snedecor et al., 1967).

\section{Thin Layer Chromatography}

In order to detect cholesterol oxidation products, thin layer chromatography (TLC) was performed with pre-coated Silica Gel $60 \mathrm{~F}_{254}$ plates (Merck). Incubation products were separated with a mixed solvent system [chloroform:diethyl ether $(60: 40)]$ at room temperature and spots were made visible by spraying with 2 ', 7 'dichlorofluorescein and viewed under ultra-violet light (254nm). Standards of cholesterol and possible oxidation byproducts (5-cholesten-3-one, 4-cholesten-3one, 25-hydroxycholesterol and 7-ketocholesterol) were also spotted.

\section{Gas Chromatography-Mass Spectroscopy (GC-MS)}

The identification of by-products was confirmed using gas chromatographic retention times in conjunction with their mass spectra. Incubation products were extracted twice with diethyl ether $(2 \mathrm{~mL}$ and $1 \mathrm{~mL})$, the extracts evaporated to 
dryness and the residues dissolved in chloroform $(500 \mu \mathrm{L})$. Samples $(1 \mu \mathrm{L})$ were then taken for GC-MS using a Hewlett Packard 5890 gas chromatograph fitted with a Hewlett Packard 5971A Mass Selective Detector (MSD). The column and chromatographic conditions were the same as for cholesterol determination.

\subsection{Microbiological Treatment of Egg Yolk and Milk Cholesterol}

\section{Solvents}

- Isopropanol anhydrous, Sigma Chemical Co.

\section{Media}

- $\quad$ Liquid minimal medium $(\mathrm{pH} 7)$ contained $(\mathrm{g} / \mathrm{L})$ :

ammonium nitrate $\left(\mathrm{NH}_{4} \mathrm{NO}_{3}\right), 1.0$

di-potassium hydrogen orthophosphate $\left(\mathrm{K}_{2} \mathrm{HPO}_{4}\right), 0.25$

magnesium sulphate $\left(\mathrm{MgSO}_{4} .7 \mathrm{H}_{2} \mathrm{O}\right), 0.001$

ferrous sulphate hepta-hydrate $\left(\mathrm{FeSO}_{4} .7 \mathrm{H}_{2} \mathrm{O}\right), 0.001$

yeast extract, Oxoid L21, 5.0

- Minimal medium agar was prepared from liquid minimal medium containing $1.5 \%$ agar, Oxoid L13.

- Neutralised bacteriological peptone, Oxoid L34: 0.1\% in distilled water.

- $\quad$ Nutrient agar, Oxoid CM3 containing 0.4\% yeast extract, Oxoid L21.

- $\quad$ Nutrient broth, Oxoid CM1 containing 0.4\% yeast extract, Oxoid L21.

- $\quad$ Plate count agar, Oxoid CM 325. 


\section{Cultures and Media}

Cholesterol degrading strains of Rhodococcus species were obtained from the Japan Collection of Microorganisms (JCM) as lyophilised preparations. The strains studied were, Rhodococcus equi No. 23 (JCM 6819) isolated from butter, Rhodococcus erythropolis No. 3 (JCM 6823) isolated from pork fat, Rhodococcus species No. 13 (JCM 6830) isolated from chicken fat, and Rhodococcus species No.15 (JCM 6831) isolated from chicken fat, as described by Aihara et al. (1986).

Stock cultures were prepared by growth on nutrient agar and re-suspension of cells in Nutrient Broth (Oxoid Australia) containing glycerol (15\%w/v) and maintained at $-20^{\circ} \mathrm{C}$. Routine sub-culture was performed in liquid minimal medium at $30^{\circ} \mathrm{C}$ for $2 \mathrm{~d}$.

\section{Growth Curves for Microorganisms}

A growth curve was established for each microorganism, so that cells could be harvested for cholesterol degradation studies at the desired stage of growth.

Stock culture $(250 \mu \mathrm{L})$ was inoculated into $10 \mathrm{~mL}$ nutrient broth and incubated at $30^{\circ} \mathrm{C}$ with mechanical shaking (220cycles/min). Turbidity readings at $530 \mathrm{~nm}$ were taken at various time intervals until the stationary phase of the growth cycle was reached. 
Detection of COD Activity

Level of Isopropanol Tolerance by Rhodococcus sp.

To measure the cholesterol degrading ability of the microorganisms studied it was necessary to determine cell viability at varying strengths of isopropanol necessary to dissolve pure cholesterol in liquid minimal medium.

Microorganisms ( $10^{6}$ cells) were inoculated into liquid minimal medium $(1 \mathrm{~mL})$ containing varying levels of isopropanol: 5,10, 20 and 50\%. Liquid minimal medium provided a minimal requirement of minerals and salts for growth by each culture. Media solutions were incubated at $30^{\circ} \mathrm{C}$ with mechanical shaking for $24 \mathrm{~h}$. Media solutions containing 0\% isopropanol were also included and used as controls.

Detection of COD Activity on Agar Medium

Stock culture $(250 \mu \mathrm{l})$ was grown to mid log phase in $10 \mathrm{~mL}$ Nutrient Broth containing $0.4 \%$ yeast extract at $30^{\circ} \mathrm{C}$, using an orbital shaker set at 220 cycles/min. Aliquots $(1 \mathrm{~mL})$ of cell cultures were further diluted in minimal medium broth $(100 \mathrm{~mL})$.

The four strains of Rhodococcus species were inoculated $(10 \mu \mathrm{L})$ on minimal agar plates with and without added cholesterol at a level of $0.4 \%$, and incubated at $37^{\circ} \mathrm{C}$ for $7 \mathrm{~d}$. Cholesterol was dissolved in a $1 \mathrm{~mL}$ solution of $5 \%$ isopropanol in minimal medium prior to addition to minimal medium agar. Rhodococcus equi No. 23 was 
also inoculated on minimal medium agar containing egg yolk at a level of $33 \%$ (equivalent to $0.4 \% \mathrm{egg}$ cholesterol). Cultures were examined for a halo of cholesterol degradation.

\subsubsection{Cholesterol Degradation in Milk}

UHT milk sterilised at ultra high temperatures was aseptically diluted, 1 in 2, 1 in 4 and 1 in 8 in liquid minimal medium. Since the cholesterol level in the undiluted milk was $1.42 \mathrm{mg} / \mathrm{mL}$, the diluted cholesterol levels were $0.71,0.35$ and 0.17 $\mathrm{mg} / \mathrm{mL}$, respectively.

Undiluted milk and milk dilutions ( $1 \mathrm{~mL}$ ) were inoculated with cells of $R$. equi No. $23\left(10^{4} \mathrm{cfu}\right)$ and incubated at $37^{\circ} \mathrm{C}$ using an orbital shaker incubator set at 220 cycles/min. Cholesterol was determined at day 7. Controls incubated for $7 \mathrm{~d}$ and non-incubated controls were also included. The analysis was replicated five times on the same day. The data was analysed using a two-tailed Student's t-test for the comparison of the means of two independent samples $(\mathrm{df}=8)$. Cholesterol degradation was expressed as \% reduction.

Milk diluted 8 times (10mL) was inoculated with cells of R.equi No. $23\left(10^{7} \mathrm{cfu}\right)$ previously induced $(10 \mu \mathrm{L} / \mathrm{mL})$ in the presence of milk cholesterol $(0.19 \mathrm{mg} / \mathrm{mL})$ at $37^{\circ} \mathrm{C}$ for $7 \mathrm{~d}$ to produce enzymes necessary for the cholesterol degradation. Cultures were incubated in sterile wide-mouthed glass bottles with screw caps for $7 \mathrm{~d}$ at $37^{\circ} \mathrm{C}$ using an orbital incubator shaker set at $220 \mathrm{cycles} / \mathrm{min}$. Milk was also inoculated with non-induced cells and incubated as described earlier. A sample 
( $1 \mathrm{~mL}$ ) was aseptically withdrawn at 03,5 and $7 \mathrm{~d}$ to measure cholesterol. Incubated and non-incubated controls without cholesterol were also included.

The analysis was not replicated. Residual cholesterol was expressed as \% reduction.

\subsubsection{Cholesterol Degradation in Egg Yolk}

Egg yolk, prepared by removing yolk from raw eggs under sterile conditions, was aseptically added to minimal medium $(10 \mathrm{~mL})$ at varying levels of approximately $0.5,0.27$ and $0.16 \mathrm{~g}$. The corresponding cholesterol levels were $0.57,0.32$ and $0.19 \mathrm{mg} / \mathrm{mL}$, respectively.

Yolk sample (10mL) was inoculated with non-induced and induced cells of $R$. equi No. 23 (previously grown in $0.19 \mathrm{mg} / \mathrm{mL}$ yolk cholesterol) as described above for the milk test system. Samples $(1 \mathrm{~mL})$ were aseptically removed at $0,3,5$ and $7 \mathrm{~d}$ for analysis of cholesterol. Incubated and non incubated controls were also included. Residual cholesterol was expressed as \% reduction.

\subsubsection{Cholesterol Degradation Products}

The halo section on the plate of $R$. equi No. 23 grown on yolk-containing medium was removed and the cholesterol extracted with diethyl ether and quantitatively analysed by gas chromatography. The diethyl ether extract was also analysed for possible degradation products by gas chromatography-mass spectrometry and thin-layer chromatography. An investigation of the degradation products present in incubated 
egg yolk cultures was also performed using thin-layer chromatography and gas chromatography-mass spectrometry. 


\section{CHAPTER 3}

Optimisation of Cholesterol Assay 


\subsection{Introduction}

There have been numerous methods reported for assaying cholesterol in foods. The classical methods for the determination of dietary cholesterol are based on the Liebermann-Burchard colour reaction or the Zlatkis et al. (1953) procedure (Ulberth and Reich, 1992). Other methods include gravimetry, titrimetry, thinlayer chromatography and radioassay (Beyer et al., 1989b; Horvath, 1966). A commonly used method is the enzymatic determination of cholesterol using cholesterol oxidase (Masoom and Townsend, 1985; Karkalas et al., 1982; Kushiro et al., 1982), that usually involves an organic extraction (Beyer et al., 1989b; Warren et al., 1988). A more recent process describes the use of a surface adsorbent which selectively adsorbs sterols from fluid mixtures, such as egg yolk (Klemann and Finley, 1991).

Some of these methods, however, are not strictly specific for cholesterol and have overestimated the true content due to interfering non-cholesterol substances found in various foods (Beyer and Jensen, 1989b; Swift, 1984; Kushiro et al., 1982; Heftmann and Hunter, 1979; Weiss et al., 1964). Chromagens of non-cholesterol substances developed by colorimetric methods are often similar to that developed using cholesterol (Beyer and Jensen, 1989b; Swift, 1984; Kushiro et al., 1982), and sterols closely related structurally to cholesterol react almost as well as cholesterol with the cholesterol oxidase enzyme (Swift, 1984; Kushiro et al., 1982).

The higher values obtained by the colorimetric methods on unsaponifiable yolk extract have been suggested to be due to interference from polyunsaturated fats 
(Weiss et al., 1964; Heftmann and Hunter, 1979). The cholesterol content of eggs laid by hens fed $30 \%$ safflower oil or linseed oil was overestimated by $36 \%$ and 42\% respectively, when the direct Zlatkis et al. (1953) method for measuring cholesterol was used. For unsaponifiable yolk extracts cholesterol increased by $46 \%$ and by as much as $26 \%$ in saponifiable extracts (Beyer and Jensen, 1989b). Overestimation of the cholesterol content of eggs as a result of data obtained primarily by colorimetric methods (Beyer and Jensen, 1989b) may lead to errors in determining the effect of egg consumption.

Enzymatic methods that use cholesterol oxidase with a high specificity for 3Bhydroxy sterol may also in some foods overestimate the cholesterol content. For example, sterols with structures very similar to that of cholesterol coexist in shellfish, thus precise cholesterol measurements in shellfish are difficult (Swift, 1984; Kushiro et al., 1982).

It follows therefore, that methods based on the separation of the cholesterol fraction from interfering chromogens and sterols prior to detection are recommended (Al-Hasani et al., 1990; Beyer and Jensen, 1989b; Heftmann and Hunter, 1979). As a result, chromatographic techniques are now preferred for dietary cholesterol analysis (Ulberth and Reich, 1992; Heftmann and Hunter, 1979).

The procedure of heating the sample with a solution of alkali in alcohol is widely used for separating sterols from total lipid extracts (Tsai et al., 1980; Kou and Holmes, 1985; Maerker and Unruh, 1986; Ramesh et al., 1979). Because the treatment results in complete hydrolysis of saponifiable lipid to yield products 
which are soluble in aqueous alkali, sterols and any other non-saponifiable lipids are therefore readily separable by extraction with an organic solvent (Martin $e t$ al., 1963; Naber and Biggert, 1985; Sugano and Watanabe, 1961).

An HPLC method used by Beyer $e$ t al. (1989) showed the cholesterol content of eggs to be much lower than previous measurements and provided a more accurate assessment of the true cholesterol intake associated with egg consumption.

Therefore, in complex biological extracts that contain not only a large excess of other lipids, but also closely related cholesterol analogues, ie., for foods known to contain a mixture of animal fats and vegetable oils, gas chromatography or high performance liquid chromatography methods of analysis are recommended (Beyer et al., 1989; Brown, 1987; Ritchie and Jee, 1985; Karkalas et al., 1982; Tu et al., 1970). Gas chromatography systems, however, have a faster recycle time than liquid chromatography systems, and the potential for more rapid analysis when an automated device is used (Beyer et al., 1989).

The internal standard procedure is widely accepted as an accurate method for quantitative analysis by gas chromatography (Kovacs, 1990; Bitman and Wood, 1980; Chicoye et al., 1968; Korahani et al., 1981; Morgan and Armstrong, 1989). The method involves the addition of an accurately known quantity of a substance (the internal standard) which is not present in the original mixture. After gas chromatography, the area of the internal standard peak is compared with the area of the peak whose concentration is to be determined. An additional gas chromatography run of a mixture containing known amounts of both the analyte 
and the internal standard is normally required to correct for differences in flame ionisation detector response between the two compounds.

Because the basis of this procedure is the ratio of two chromatographic peak areas, the determination is independent of the amount of sample injected onto the gas chromatography column.

Steroids with a free hydroxyl group usually show evidence of adsorption or dehydration when chromatographed under conditions involving relatively high temperatures $\left(280^{\circ} \mathrm{C}\right.$ to $320^{\circ} \mathrm{C}$ ) (Jennings, 1980). By reaction with a suitable silylating reagent, however, a compound with a free hydroxyl group can be converted to a trimethylsilyl (TMS) ether derivative (Kuksis et al., 1969; Gleispach, 1974). Silylation of the compound reduces loss by adsorption, improves chromatographic efficiency with resultant enhancement of resolution, and yields symmetrical Gaussian-shaped peaks (Luukkainen et al., 1961).

In a non-catalysed silylation reaction a trimethylsilyl (TMS) group is transferred from a donor molecule (silylating reagent) to a receptor molecule by a reaction mechanism which is analogous to that of acyl transfer. The TMS group is usually attached to a nitrogen or oxygen atom in the donor group, and the rate of reaction is affected both by the structure of the silylating reagent and by the structure of the receptor molecule (Chambaz and Horning, 1969). Figure 3.1 shows the silylation of cholesterol using $\mathrm{N}$-methyl-N-(trimethylsilyl)-trifluoroacetamide (MSTFA) as the TMS donor. 
<smiles>CC(C)CCC(C)C1CCC2C3CC=C4CC(O)CCC4(C)C3CCC12C</smiles>

Cholest-5-en-3B-ol

MSTFA

Cholest-5-en-3B-trimethylsilyl ether N-methyl-trifluoroacetmide (TMS ether derivative)

Figure 3.1 Conversion of cholesterol to the TMS ether derivative (Berezkin, 1983), with Nmethyl-N-(trimethylsilyl)-trifluoroacetamide (MSTFA)

The character of a molecule is altered from polar and active to non-polar and inert and is generally coupled with an increase in volatility and thermal stability, highly desirable properties for gas chromatography (Chambaz and Horning, 1969).

The rate of derivative formation often depends upon the position of substitution, and consequently vastly different rates of reaction may be observed in the formation of TMS derivatives for functional groups in hindered and relatively unhindered positions (Chambaz and Horning, 1969).

Increased public awareness to the effect of cholesterol on $\mathrm{CHD}$ and the relationship of blood cholesterol to the diet has made it necessary to have a rapid, reliable and accurate method to satisfy nutritional labelling demands (Al-Hasani et al., 1990). This study seeks to develop a rapid and efficient procedure that can be used routinely to quantify cholesterol in both egg and other cholesterol containing foods. 


\subsection{Results and Discussion}

The method described by Kovacs (1990) to determine cholesterol in pasta products was modified to measure cholesterol in a wide range of foods. The procedure was significantly improved by employing diethyl ether rather than hexane, the conversion of cholesterol to the trimethylsilyl (TMS) derivative prior to gas chromatography, and the use of either octacosan-1-ol or dihydrocholesterol, rather than cholestane, as an internal standard. The saponification procedure used ensured that total (esterified and unesterified) cholesterol was isolated and therefore gave a high recovery of cholesterol associated with lipoprotein. The smaller volumes of solvent used created limited disposal problems and silylation of the analyte gave a better resolution of chromatographic peaks.

\subsubsection{Internal standards}

The internal standards, octacosan-1-ol and dihydrocholesterol, were used for different stages of this work. The majority of the work involved in optimising GC conditions was performed using octacosan-1-ol, however the cholesterol analysis of several foods was performed using dihydrocholesterol as an internal standard. Dihydrocholesterol was also used for all of the cholesterol analyses associated with the enzymatic and microbiological cholesterol degradation studies.

Octacosan-1-ol, a long chain alcohol, is isolated from the wax found on green blades of wheat (Merck). Eluting within $2.75 \mathrm{~min}$ of cholesterol, it did not interfere with other sterols and proved a reliable and accurate internal standard to use. 
Unlike octacosan-1-ol, dihydrocholesterol has very similar chromatographic properties to those of cholesterol. It also has similar solubilities to cholesterol in alcohol and diethyl ether. Though dihydrocholesterol and cholesterol are two very closely related substances eluting within $18 \mathrm{~s}$ of each other, near baseline separation was achieved under the chromatographic conditions used (Figure 3.2).

It was established that interference from other sterols did not occur in GC analysis under the conditions employed since most other sterols eluted at longer retention times than octacosan-1-ol, dihydrocholesterol and cholesterol.

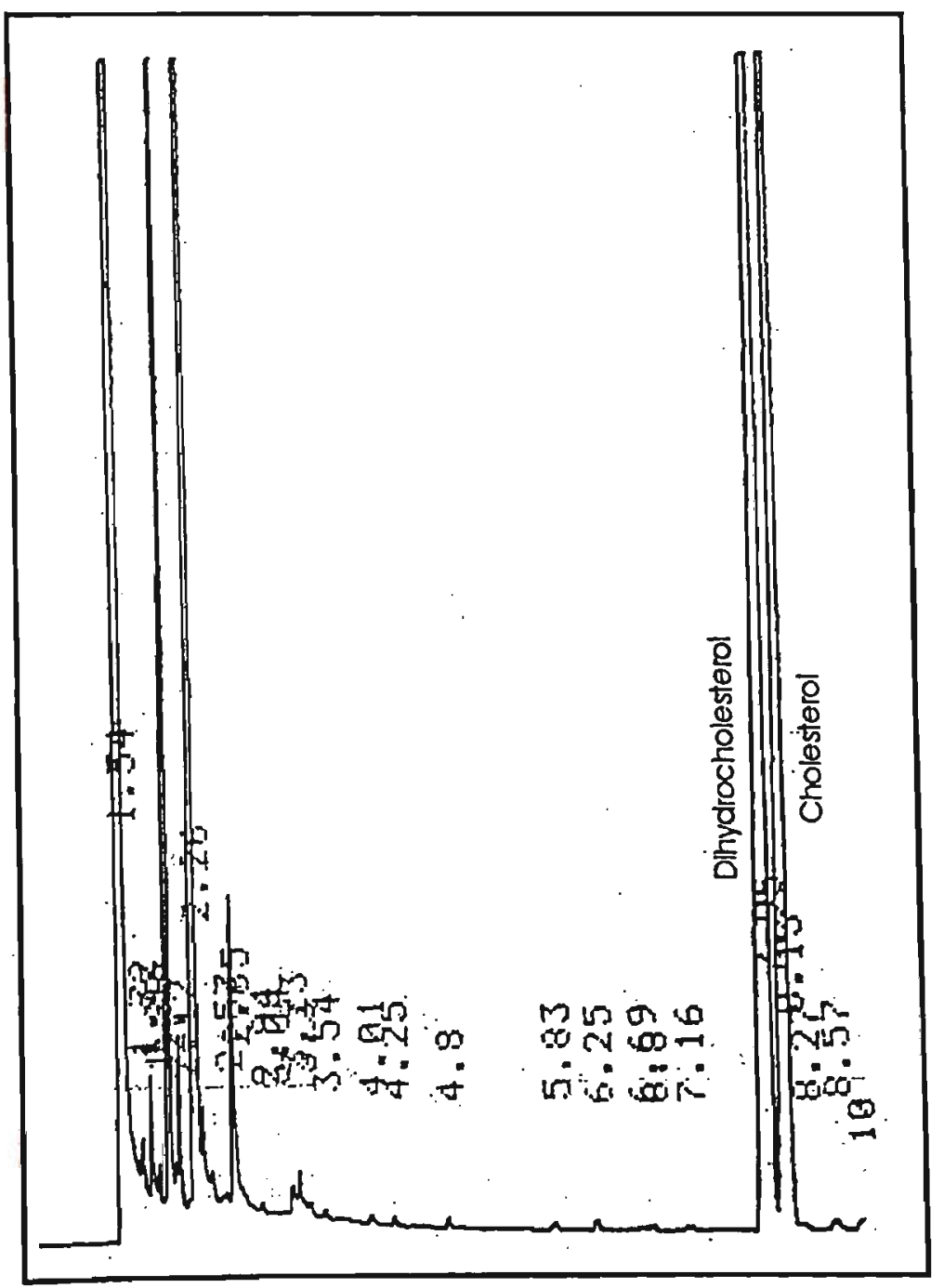

Figure 3.2 Chromatogram showing-near baseline separation achieved between dihydrocholesterol (1) and cholesterol (2) under the chromatographic conditions used. 
When the two internal standards were used to measure the cholesterol in egg yolk, the cholesterol values obtained were not significantly different at the $5 \%$ level of significance $(\mathrm{p}<0.05)$ (Table 3.1).

Table 3.1 The analysis of cholesterol in egg yolk using either octacosan-1-ol or dihydrocholesterol as the internal standard.

\begin{tabular}{ll}
\hline Internal Standard & Cholesterol $(\mathrm{mg} / \mathrm{g})^{*}$ \\
\hline Dihydrocholesterol & $12.04 \pm 0.08$ \\
Octacosan-1-ol & $12.00 \pm 0.23$ \\
\hline mean \pm standard error; $\mathrm{n}=4$. &
\end{tabular}

However, since the chromatographic and solubility properties of dihydrocholesterol are so similar to cholesterol and also because it is relatively inexpensive, readily available and chemically and thermally stable, it is an ideal internal standard for cholesterol analysis and was used for the bulk of the cholesterol analysis reported in this thesis.

Egg yolk was suspended in a buffer medium for all the cholesterol analyses conducted since a greater amount of the cholesterol was extracted than when the yolk was assayed fresh. The cholesterol value $(12.32 \mathrm{mg} / \mathrm{g} \pm 0.17)$ was significantly different $(\mathrm{p}<0.05)$ to that obtained when the same egg yolk was assayed fresh $(12.04 \mathrm{mg} / \mathrm{g} \pm 0.08)$. Cholesterol appeared to be more accessible to solvent extraction when homogenously suspended in a liquid medium. 


\subsubsection{Silylation}

The hydroxyl groups of cholesterol, octacosan-1-ol and dihydrocholesterol are all sterically unhindered and readily derivatised with $\mathrm{N}$-O-bis-(trimethylsilyl)trifluoroacetamide (BSTFA) and $\mathrm{N}$-methyl-N-(trimethylsilyl)-trifluoroacetamide (MSTFA).

Derivatisation improved the peak shapes of cholesterol, octacosan-1-ol (Figure 3.3) and dihydrocholesterol, and also increased their detector response. Also, because of the similarity in retention time of cholesterol and dihydrocholesterol, the improved peak shape associated with derivatisation significantly enhanced the resolution of these two compounds (Figure 3.2).
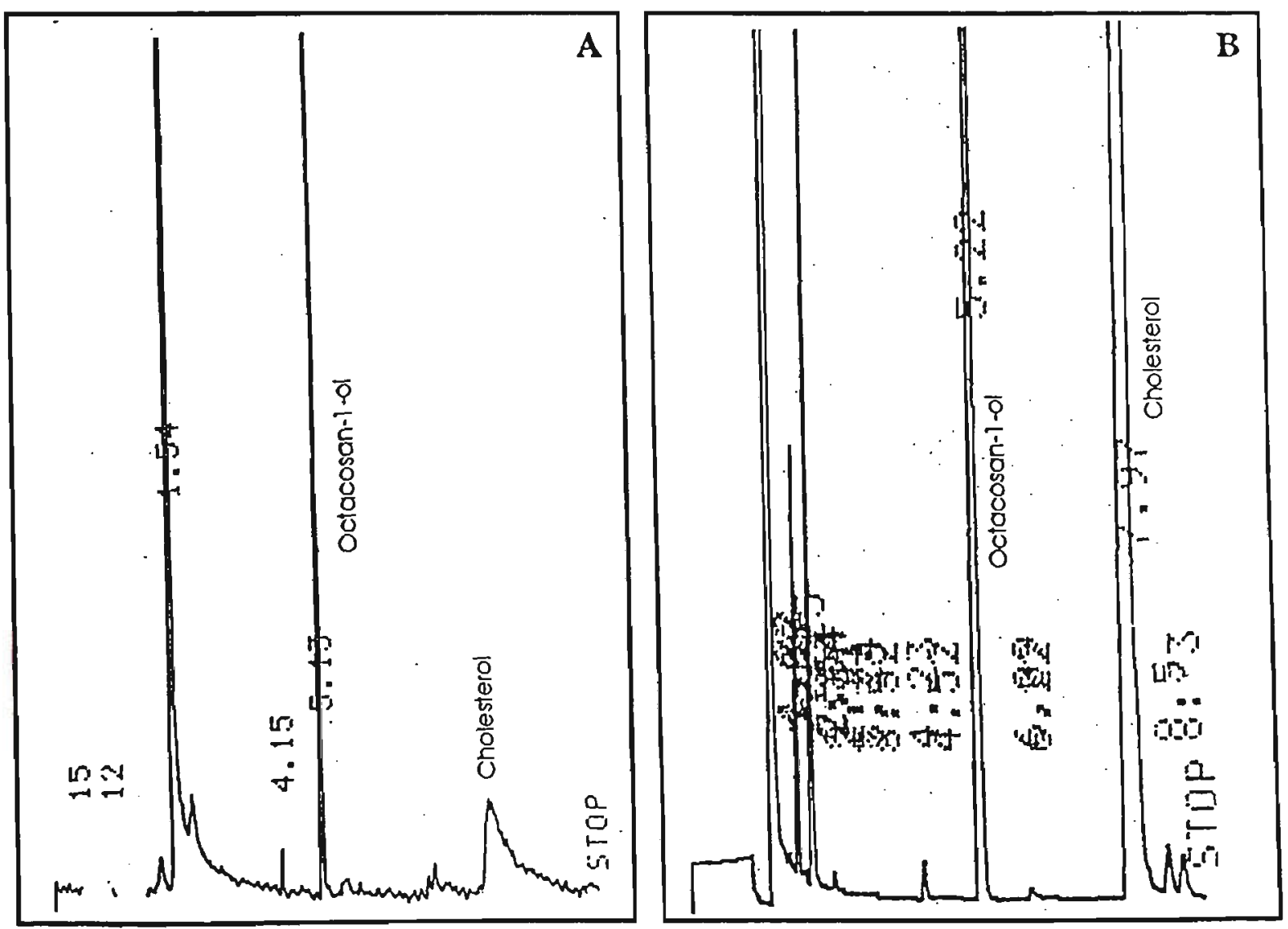

Figure 3.3 Gas chromatograms under identical conditions showing (A) peak shape quality of underivatised octacosan-1-ol and cholesterol, and (B) improved peak shape quality after derivatising with MSTFA. 


\section{Silylation Reaction Time Study}

To establish appropriate conditions for derivative formation prior to GC, the relative rates of reaction of hydroxyl groups with the two silylating reagents, N-Obis-(trimethylsilyl)-trifluoroacetamide (BSTFA) and N-methyl-N-(trimethylsilyl)trifluoroacetamide (MSTFA), were examined under various reaction conditions. This study indicated that these reactions can be accelerated by heating (Chambaz and Horning, 1969). Silylation was considered complete when none of the unsilylated components of the standard mixture could be detected in the chromatogram.

The ease of derivative formation varied greatly between cholesterol and octacosan1-ol. Although BSTFA has the ability to release two TMS groups, rapid silylation at room temperature was not observed. Complete silylation of the reaction mixture required up to $50 \mathrm{~min}$ at $100^{\circ} \mathrm{C}$ (Figure 3.4) compared to $15 \mathrm{~min}$ with MSTFA. Excess MSTFA $(100 \mu \mathrm{l})$ gave complete silylation of the standard mixture at room temperature, in less than one minute, but for an egg yolk extract sample, complete silylation required $15 \mathrm{~min}$ at $100^{\circ} \mathrm{C}$.

Both reagents form silyl derivatives with the sterically non-hindered hydroxyl groups, but the high silyl-donor effectiveness of MSTFA made it an excellent reagent for the silylation of cholesterol and the internal standards. 


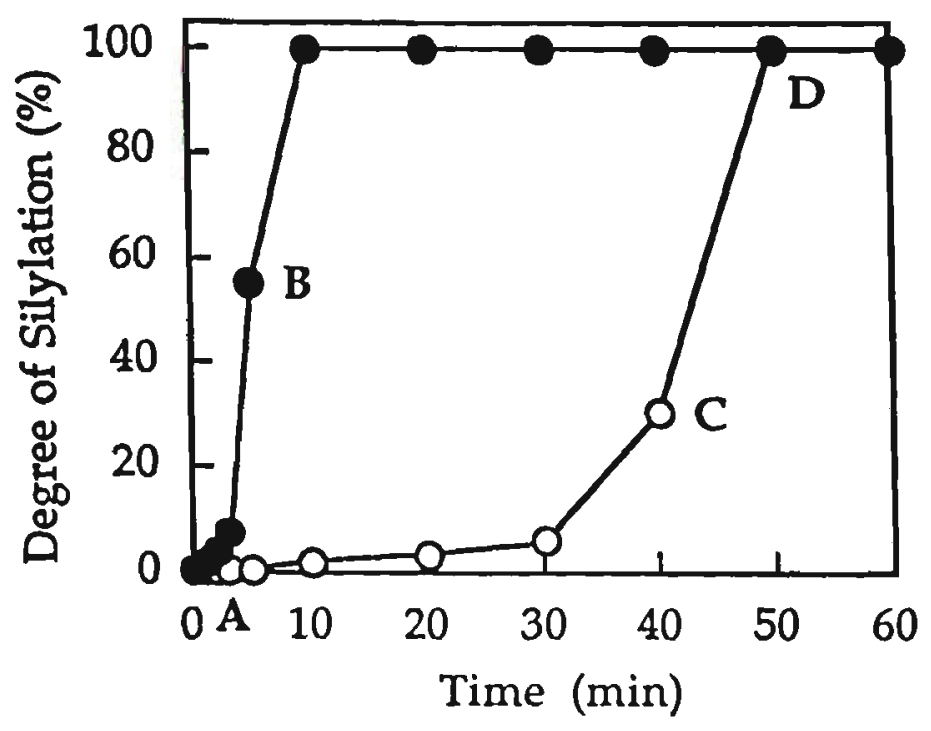

octacosan-1-ol $\longrightarrow$ cholesterol
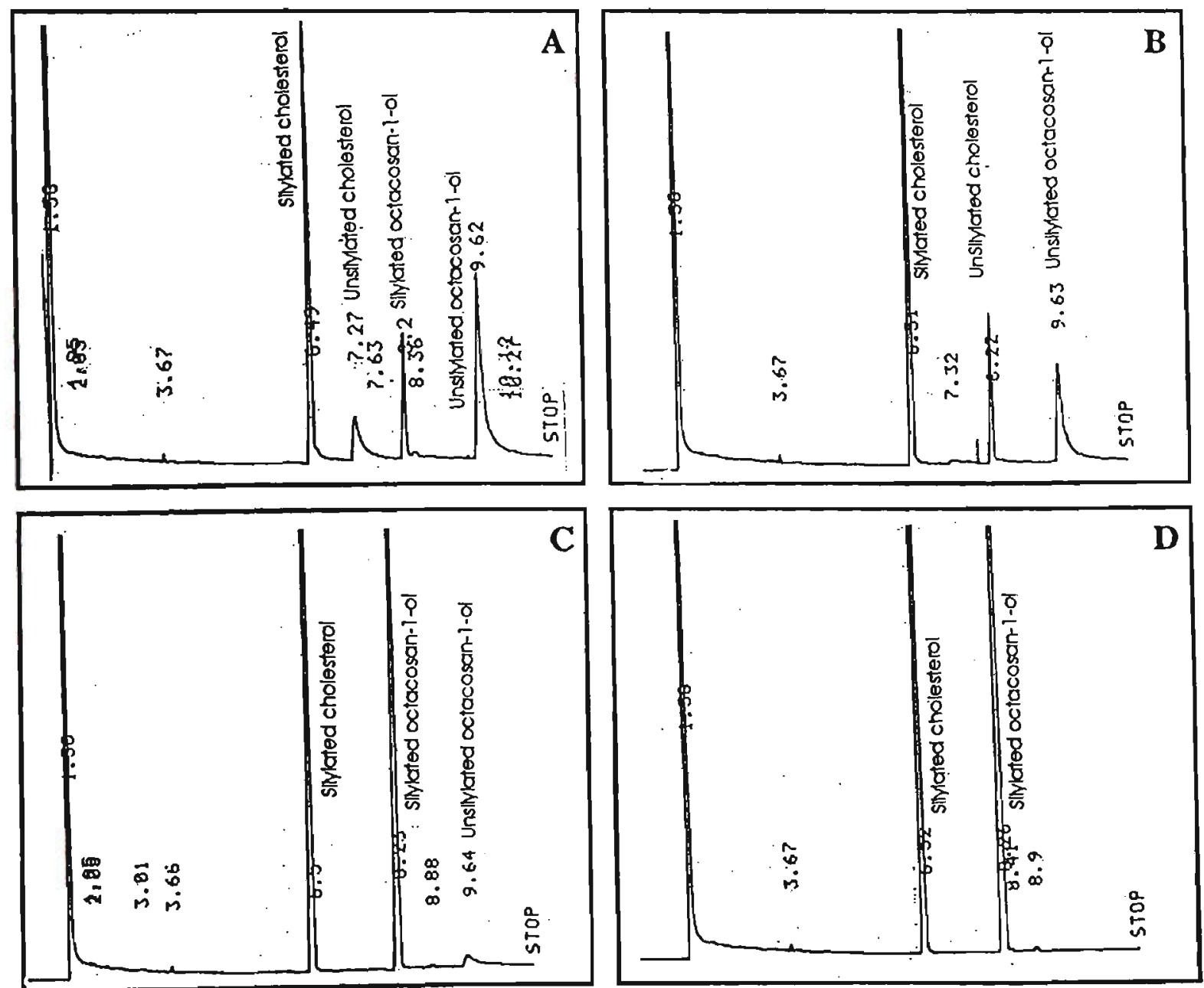

Figure 3.4 The rate of derivative formation in a mixture containing equal amounts of octacosan$1-01$ and cholesterol using BSTFA at $100^{\circ} \mathrm{C}$, and gas chromatography traces of the reaction mixtures after (A) $2 \mathrm{~min},(B) 5 \mathrm{~min}$, (C) $40 \mathrm{~min}$ and (D) $50 \mathrm{~min}$. 


\subsubsection{Position of Capillary Column in Varian Gas Chromatograph Injector}

Peak quality as indicated by both Gaussian symmetry and magnitude of area was found to be influenced by the position of the inlet end of the capillary column in the glass-lined metal tube leading down from the injector into the gas chromatograph oven. The peak quality was judged optimal when the peak height ratio and peak area ratio obtained from the cholesterol/octacosan-1-ol standard were both nearly unity; this occurred when approximately $8 \mathrm{~cm}$ of the capillary column was inserted into the glass liner of the injector port (Figure 3.5).

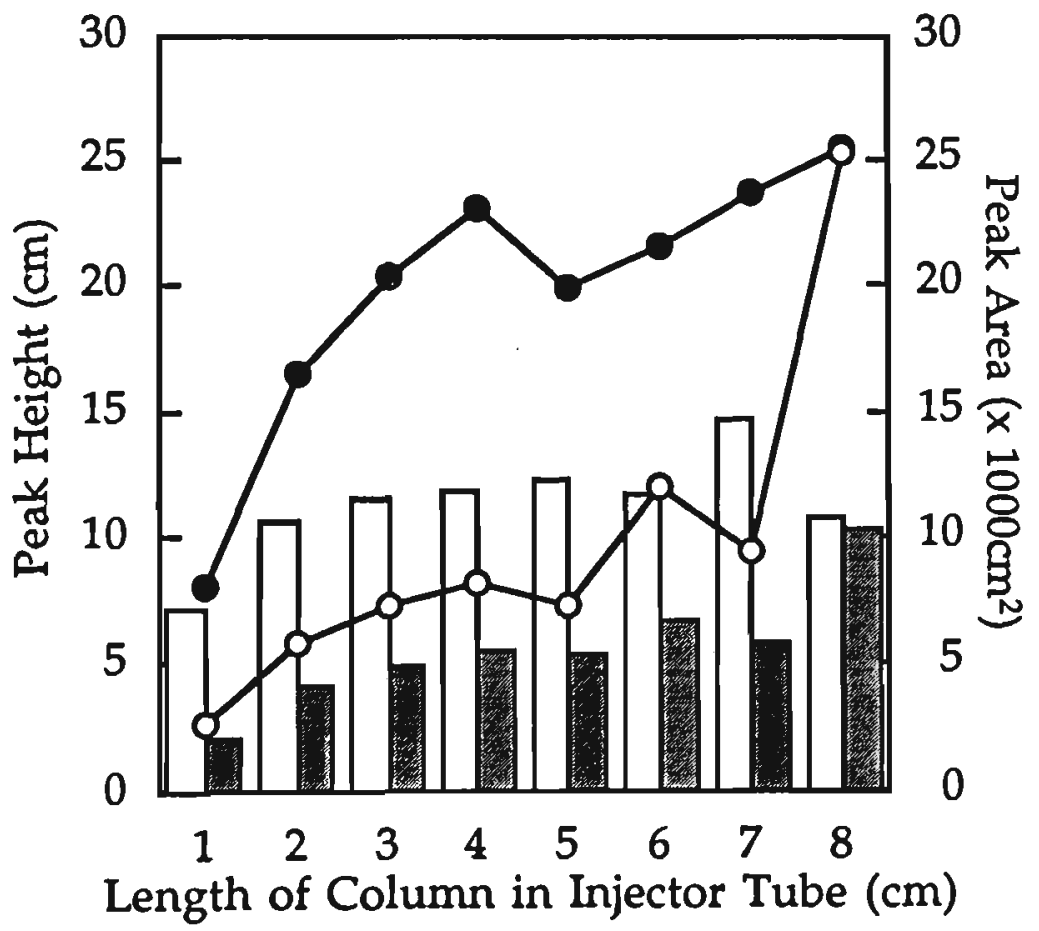

Cholesterol Peak Height
Cholesterol Peak Area

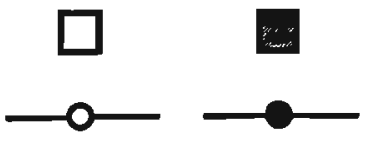

Octacosan-1-ol Peak Height

Octacosan-1-ol Peak Area

Figure 3.5 The effect on peak quality by varying the length of capillary column inserted into the injector tube of a gas chromatograph: measured by the ratio of peak area between cholesterol and octacosan-1-ol and the peak height of the same two compounds. 
When the length of the column inserted was less than $8 \mathrm{~cm}$ or greater than $8 \mathrm{~cm}$, bad tailing on the trailing edge of peaks, and significant differences in peak heights was observed (Figure 3.5).

\subsubsection{Relative Response Factors}

The response of a detector is the quantity of a signal generated by a given amount of the sample. The general rule is that equal amounts of components with similar functional groups give equal responses, however, correction factors are usually necessary when different functional groups are involved.

The relative response factor, measured as the peak area of the internal standard relative to cholesterol, was $1.064(n=8)$ and $1.022(n=4)$ for dihydrocholesterol and octacosan-1-ol, respectively. The respective relative response factor was then incorporated as a correction factor into all cholesterol determinations.

\subsubsection{Determination of Cholesterol}

The identification of cholesterol and dihydrocholesterol in gas chromatography traces was facilitated by comparison of retention times with those of known standards chromatographed under identical conditions. Quantification of cholesterol was achieved by comparison of the areas of the cholesterol and dihydrocholesterol peaks and the application of a small factor to recognise a slight difference in the response of each of these compounds in the flame ionisation detector. 


\subsubsection{Reproducibility and Recovery}

Reproducibility data collected for egg yolk for each assay procedure yielded markedly different results; the modified procedure gave a higher cholesterol value, along with a lower coefficient of variation, than the Kovacs procedure (Table 3.2).

Table 3.2 Comparisons of extraction by the modified and original procedures in egg yolk.

\begin{tabular}{|c|c|c|c|}
\hline \multirow[t]{2}{*}{ Procedure } & \multicolumn{2}{|c|}{ Reproducibility } & \multirow{2}{*}{$\begin{array}{l}\text { \% Recovery } \\
\text { mean } \pm \text { s.e. }\end{array}$} \\
\hline & mean \pm s.e. & C. $\mathrm{v}$. & \\
\hline \multicolumn{4}{|c|}{ mg cholesterol/g yolk } \\
\hline Modified ${ }^{1}$ & $16.10 \pm 0.06$ & $1.1 \%$ & $103.1 \pm 1.5$ \\
\hline $\begin{array}{l}\text { Original }{ }^{2} \\
\text { C. V.=coeffic } \\
1_{n=8} \\
{ }^{2} n=7\end{array}$ & $10.07 \pm 0.11$ & $3.1 \%$ & $71.7 \pm 2.0$ \\
\hline
\end{tabular}

The amount of cholesterol recovered by the modified procedure, when $10 \mathrm{mg} / \mathrm{g}$ of cholesterol was added to a series of egg yolk samples, was $10.31 \pm 0.15 \mathrm{mg} / \mathrm{g}$, however, only $7.17 \pm 0.20 \mathrm{mg} / \mathrm{g}$ was recovered by the Kovacs procedure. The Kovacs procedure was, therefore, extracting only about two thirds of the available cholesterol as shown by both the reproducibility and recovery data.

For uncooked noodles, a lower coefficient of variation and a higher \% recovery was obtained with the modified procedure, while the original procedure gave a higher coefficient of variation and a lower recovery. The difference \pm s.e. for the 
recoveries using the original and modified procedures were $2.52 \pm 0.04 \mathrm{mg} / \mathrm{g}$ and $1.96 \pm 0.08 \mathrm{mg} / \mathrm{g}$, respectively.

\subsubsection{The Determination of Cholesterol in Multi-component Foods}

When comparing the method of Kovacs (1990) with a laboratory kit used for the enzymatic determination of cholesterol, Karkalas et al. (1982) found very good agreement between the test materials analysed. All of the test materials assayed in the Karkalas et al. investigation, however, contained less than $2 \mathrm{mg}$ cholesterol/g sample. The reproducibility and recovery for cholesterol extracted from pasta samples with low cholesterol $(1.52 \mathrm{mg} / \mathrm{g})$ by either method in this study and those reported by Karkalas et al. (1982), are in contrast with those obtained from egg yolk with high cholesterol $(16.10 \mathrm{mg} / \mathrm{g})$. It is suggested that the accuracy of the Kovacs method is affected by the level of cholesterol in each type of food.

To assess the suitability of the modified gas chromatographic method for measuring cholesterol in foods other than egg, the cholesterol content of several foods available in Melbourne markets was investigated (Table 3.3). The chromatograms for these foods gave excellent baseline separation without interferences and triplicate samples of the same food type indicated repeatability between assays.

The Italian egg spaghetti had a cholesterol content in the range $0.15-0.79 \mathrm{mg} / \mathrm{g}$ while egg noodles had $0.93-1.42 \mathrm{mg} / \mathrm{g}$. The high level of cholesterol found in egg noodles was presumably due to their being prepared with egg yolk, while the Italian pasta was made with whole egg. Some egg rolls had very high cholesterol 
contents $(1.83-3.84 \mathrm{mg} / \mathrm{g})$. In meat-based products, ham products had a cholesterol level similar to pork cakes or pork loafs $(0.63-0.71 \mathrm{mg} / \mathrm{g}$ compared with $0.70-0.86 \mathrm{mg} / \mathrm{g}$, respectively). A similar cholesterol level was also found among fish balls and cheddar cheese $(0.99 \mathrm{mg} / \mathrm{g}$ and $1.01 \mathrm{mg} / \mathrm{g}$, respectively). Chicken liver, chicken paste and prawn had the highest cholesterol contents among the products investigated (Table 3.3).

Table 3.3 Cholesterol (mg/g士s.e.*) content of various foods.

\begin{tabular}{|c|c|c|c|}
\hline MEAT PRODUCT & $\mathrm{mg} / \mathrm{g} \pm$ s.e. & EGG PRODUCT & $\mathrm{mg} / \mathrm{g} \pm$ s.e. \\
\hline Fish Balls & $0.99 \pm 0.04$ & $\begin{array}{l}\text { TAK ON ASIAN FOOD } \\
\text { Pure Egg Noodle }\end{array}$ & $1.42 \pm 0.05$ \\
\hline Pork Loaf & $0.86 \pm 0.004$ & MAI HONG Egg Noodle & $1.65 \pm 0.08$ \\
\hline Pork Cake & $0.70 \pm 0.03$ & INDO CHINA Chow Mein & $0.93 \pm 0.03$ \\
\hline $\begin{array}{l}\text { DON SMALLGOODS DELI SUPREME } \\
\text { Premium Unsmoked Ham }\end{array}$ & $0.63 \pm 0.01$ & PHOENIX Egg Rolls & $1.83 \pm 0.15$ \\
\hline CASTLE Strasberg & $0.67 \pm 0.02$ & KHONG GUAN Egg Rolls & $3.84 \pm 0.24$ \\
\hline $\begin{array}{l}\text { HERITAGE PATE } \\
\text { Chicken Liver with Brandy }\end{array}$ & $2.75 \pm 0.2$ & VETTA Egg Spaghetti & $0.23 \pm 0.01$ \\
\hline REGAL PATE Grand Manier & $2.63 \pm 0.19$ & $\begin{array}{l}\text { SAN REMO } \\
\text { Vegeroni Egg Spaghetti }\end{array}$ & $0.15 \pm 0.01$ \\
\hline Bacon Meat & $0.82 \pm 0.04$ & GUZZIS Tagliolini & $0.79 \pm 0.01$ \\
\hline Bacon Fat & $1.29 \pm 0.03$ & Prawn & $2.49 \pm 0.09$ \\
\hline Chicken Fillet & $0.74 \pm 0.05$ & & \\
\hline Chicken Liver & $4.25 \pm 0.04$ & OTHER & $\mathrm{mg} / \mathrm{g} \pm$ s.e. \\
\hline Lean Topside & $0.76 \pm 0.02$ & MAI HONG Won Ton Skin & $0.14 \pm 0.04$ \\
\hline Beef Loin & $0.83 \pm 0.02$ & Cheddar Cheese & $1.01 \pm 0.08$ \\
\hline
\end{tabular}

* $n=3$ for all foods analysed

The method demonstrated a capacity to extract cholesterol in both non-egg and egg-containing foods with different and complex matrixes, and the cholesterol level extracted did not appear to be affected by the level of cholesterol with as little as $0.23 \mathrm{mg} / \mathrm{g}$ cholesterol and up to $16 \mathrm{mg} / \mathrm{g}$ for egg yolk extracted. 


\title{
CHAPTER 4
}

\section{Degradation of Egg Yolk Cholesterol using}

\author{
Purified Enzymes
}




\subsection{Introduction}

Enzymes are proteins containing the same amino acids found in other proteins, and like other proteins have a three-dimensional structure that represents the conformation of least energy content. Enzymes function as catalysts, ie., they speed up chemical reactions which would otherwise proceed slowly, or not at all (Dziezak, 1991). This is the basis for enzyme function; the spatial arrangement of amino, carboxyl, and other bonding groups increases catalytic effectiveness by orders of magnitude (McGilvery, 1979). Reaction times for industrial enzymes vary from a few minutes to several days (Dziezak, 1991).

\subsubsection{Enzymatic Reactions}

An enzymatic reaction proceeds through at least three stages (McGilvery, 1979):

1. the formation of a complex between enzyme and substrate (ES),

2. the conversion of this complex to an enzyme-intermediate complex (EI), and

3. further conversion to a complex between enzyme (E) and product $(P)$ that can dissociate, releasing both the product and the regenerated active enzyme:

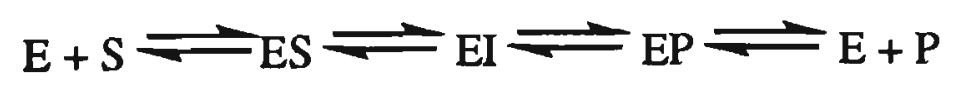

Enzymes owe their peculiar catalytic effectiveness to a combination of specific binding and the presence of catalytic groups. For example, many amino acid side chains are capable of donating or accepting protons so as to act as general acid or general base catalysts. Other groups, such as hydroxyl or amino groups, may act as nucleophiles (donate electrons to bond to other groups with vacant orbitals) 
(McGilvery, 1979) and moieties other than amino acids in the protein chains of enzymes, such as vitamins and metal ions, may also be involved in the catalytic process (Williams, 1969).

The binding and catalytic groups of the enzyme combine with the substrate in such a way that the intermediate-enzyme complex has a lower energy content than the complexes of either the substrate or the product, with the enzyme. The activation energy for formation of the intermediate-enzyme complex is therefore much lower than the activation energy for the reaction in the absence of enzyme (McGilvery, 1979).

\subsubsection{Enzyme Specificity}

Enzymes are highly specific in binding particular substrates, because the amino acid residues in the binding site closely fit only a few compounds, in some cases perhaps only one (McGilvery, 1979). Therefore, changes in the activity of particular enzymes will affect only a few compounds, an important feature of biological control (McGilvery, 1979).

The specificity of enzyme reactions is a very significant factor when evaluating enzymatic processes versus conventional chemical processes. Because of the specificity of enzymes, large amounts of compounds can be broken down or synthesised with minimum formation of byproducts. Enzymes also permit reactions to take place under mild operating conditions of temperature, pressure and $\mathrm{pH}$, which generally means reduced energy costs. Further, low usage levels make the use of enzymes economical as well as practical (Driezak, 1991). 


\subsection{Conversion of Cholesterol in Food to Coprostanol by Cholesterol Reductase}

\subsubsection{Introduction}

Cholesterol reductase (CR) is an enzyme that transforms cholesterol into coprostanol, an innocuous compound that is believed to remain inert as it passes through the digestive system (Best, 1989). The reductase enzyme was discovered in certain green plant parts and is known to be present in several bacteria that commonly inhabit the digestive tract of animals (Beitz et al., 1990).

A patented process of concentrating cholesterol reductase from an homogenate of cucumber plant leaves has been described (Beitz et al., 1990). The process involves homogenising the green plant parts, separating the fibrous materials from the crude homogenate, and concentrating the homogenate to provide a cell-free, cytosolic, cholesterol reductase-enriched preparation, or ultimately a cholesterol reductase purified to homogeneity.

The authors of the patent reported a reduction in cholesterol in food substances after treatment with the CR-enriched preparation, and a cholesterol reduction in animals to be used to provide meat after intravenous administration of a CRenriched preparation an hour prior to slaughter. The reduction in animals was described as a $50 \%$ reduction "most of the time" and a $25 \%$ reduction "all of the time" (Beitz et al., 1990). The same researchers stated that the enzyme can also be added to ground preparations of meats. 
To reduce cholesterol levels, homogenised milk can be treated directly with the CR-enriched preparation or alternatively passed through an enzyme-supporting inert material. Intact whole eggs can be treated by injecting $\mathrm{CR}$ enzyme into the yolk and broken egg mixtures can be treated directly with the CR enzyme (Beitz et al., 1990).

Cholesterol reductase activity has also been measured in Eubacterium species Strain HL (Freier et al., 1992). The culture bacteria could be redesigned so that they produce their own cholesterol reductase activity (Best, 1989). For example, by genetically altering the starter cultures the bacteria could produce cholesterol reductase while cheese is aging (Hegenbart, 1989). Potential organisms from which cholesterol-reducing enzyme activities could be transplanted into cheese starter cultures have already been identified, but a redesigned dairy culture has not yet been produced nor is it known how the genes will be expressed (Best, 1989).

\subsubsection{Results and Discussion}

The patented procedure for extracting $\mathrm{CR}$ from cucumber leaves by Beitz et al., (1990) was used to investigate the cholesterol degrading ability of the cholesterol reductase enzyme in an egg yolk system. Unfortunately, although the procedure for extracting the enzymes was followed carefully, the results reported by Beitz et al. (1990) could not be repeated.

In the experiment examining the cholesterol degrading capacity of the cholesterol reductase extract with egg yolk suspension and with pure cholesterol, there was no evidence $(p>0.1)$ of any extract effect nor any interaction of extract with medium 
or incubation time. The mean cholesterol level after treatment with the extract was $0.50 \mathrm{mg} / \mathrm{g}$ (s.e. $=0.682$ ) less than the untreated sample, with a $95 \%$ confidence interval of $(-0.87,1.87)$. These limits correspond to a range extending from a $7 \%$ increase to a $15 \%$ decrease, respectively.

The cholesterol level in the yolk was much higher than in the pure cholesterol mixture $(13.79 \mathrm{mg} / \mathrm{g}$ vs $10.37 \mathrm{mg} / \mathrm{g}$, sed $=0.305, \mathrm{p}<<0.001)$. There was statistical evidence that cholesterol increased after $24 \mathrm{~h}$ in the incubator, with or without extract, (mean $=11.62 \mathrm{mg} / \mathrm{g}$ vs $9.13 \mathrm{mg} / \mathrm{g}$ for external control, sed $=0.591$ ), but this is almost certainly an artefact.

Several unsuccessful attempts were made in May, 1991 to contact Akiva Gross, author of "Enzymatic Catalysis in the Production of Novel Food Ingredients" (Food Technology, Jan 1991) in which he made reference to work being conducted with CR at Iowa State University. Faxed inquiries seeking information about the availability of pure CR in the US, and the identity of the relevant $\mathrm{CR}$ researchers were not answered. An attempt to contact Akiva Gross by telephone was also unsuccessful. Further attempts to make contact were abandoned.

It may be that cellular release of the cholesterol reductase was not achieved during the homogenisation of the cucumber leaves. Although precautions were taken to keep the homogenised slurry cool during the homogenisation process, the increasing temperature created may have resulted in the denaturation of the enzyme.

Beitz et al. (1990) measured the ability of the CR-enriched preparation to degrade cholesterol in a complex food system, such as milk, by treating milk with 
cholesterol oxidase, a model enzyme for oxidising cholesterol. The amount of cholesterol degraded by the oxidase enzyme was considered to be the same amount that would be accessible to cholesterol reductase if the milk was treated with the reductase enzyme, and so a measure of reductase degrading ability. On this basis, cholesterol oxidation revealed that up to 80 to $90 \%$ of the cholesterol in homogenised, pasteurised whole milk would be accessible to reduction to coprostanol by treatment with cholesterol reductase, and 60 to $80 \%$ in raw whole milk.

These results should be treated with suspicion however, since the ability of cholesterol oxidase to catalyse the oxidation of cholesterol in milk seems unlikely to be mirrored by the ability of cholesterol reductase to catalyse the reduction of cholesterol. The two reactions are clearly different and the accessibility of the substrate, as well as the availability of the other reagent (oxidant or reductant), may be quite different in each case.

Indeed, Dehal et al. (1988) reported a 7\% reduction in cholesterol after treating free cholesterol directly with extracellular cucumber leaf $\mathrm{CR}$ for $75 \mathrm{~min}$ at $35^{\circ} \mathrm{C}$. Since this work was performed with free cholesterol, the accessibility of cholesterol in this preparation, would presumable be described as $100 \%$, a cholesterol availability that may not be the case in more complex systems such as ground meat, milk or eggs. The cholesterol reductase activity identified in Eubacterium species Strain HL (Freier et al., 1992) was similarly not directly tested in more complex food systems. 
Cholesterol reductase extracted from other plant sources such as Alfalfa (Medico sativa) and pea (Pisum sativum) leaf converted cholesterol to coprostanol at a rate of $23 \mathrm{nmole}$ and $2.6 \mathrm{nmole} / \mathrm{h} / \mathrm{mg}$ protein, respectively, at the temperature optimum of $37^{\circ} \mathrm{C}$ (Dehal et al., 1990, 1991). Though, the cholesterol degrading ability of alfalfa and pea leaf cholesterol reductase was demonstrated, the importance of these very small conversion rates to the consumer seeking to restrict dietary cholesterol, is at best doubtful.

The difficulties that would be encountered in using $C R$ in commercial applications to reduce the cholesterol in foods would include (1) identifying commercially viable sources of the $C R$ enzyme and its associated cofactors, and (2) achieving high enzyme yields.

\subsection{Conversion of Cholesterol in Food to Cholestenone by} Cholesterol Oxidase

\subsubsection{Introduction}

Another enzyme, a cholesterol:oxygen oxidoreductase called cholesterol oxidase (COD), has been identified as demonstrating a specificity for cholesterol and it's derivatives (Smith and Brooks, 1974; Moore et al., 1977; Tomioka et al., 1976; Ikawa et al., 1979; Cheetham et al., 1982; Inouye et al., 1982; Uwajima et al., 1974; Aihara et al., 1988; Cheillan et al., 1989). 


\section{Preparation and Characterisation of COD}

COD is an inducible intracellular enzyme and can be prepared by incubating bacterial cells in a cholesterol medium (Flegg, 1973). Bacterial cells of Nocardia erythropolis have been grown in such a medium by Flegg for the production of the enzyme.

The COD produced was separated by sonication of the cholesterol medium to rupture the cells and centrifugation to sediment any residual cell matter and cholesterol. As the bacterial cells contain many other enzymes, especially enzymes acting on cholest-4-ene-3-one, the COD can be purified further by ammonium sulphate fractionation of the cell-free supernatant.

This partially purified COD mixture can then be used to determine cholesterol in serum, for example, based on the steady accumulation of cholest-4-ene-3-one in the oxidation of cholesterol by the COD enzyme.

Although, complete specificity for cholesterol is not exhibited by COD, the enzymatic technique is more specific than colorimetric cholesterol assays. COD enzyme can also be isolated from the broth filtrates of Streptomyces violascens (Fakuda et al., 1973; Tomioka et al., 1976), Brevibacterium sterolicum (Uwajima et al., 1973, 1974) and Streptoverticullim cholesterolicum (Inouye et al., 1982).

COD from Nocardia species and Pseudomonas were characterised by Richmond (1973) and Cheillan et al.(1989) respectively with a view to performing enzymatic determinations of cholesterol. 
COD from Pseudomonas fluorescens exhibited constant activity over a range of cholesterol concentrations $(2.59,5.18$ or $10.36 \mathrm{mM})$ provided that the $\mathrm{pH}$ of the medium was maintained between 5.5 and 8.5 (Cheillan et al., 1989). Enzyme that is immobilised must remain stable for a long time in the reaction medium. The effect of this prolonged incubation on the retention activity of COD was studied by Cheillan et al. (1973) and the most favourable $\mathrm{pH}$ for the conservation of this protein was found to be near the isoelectric point (pH 5 to 5.5). This could be of value especially in treatments aimed at immobilising enzymes.

The bulk of egg cholesterol is found in the low-density lipoprotein (LDL) particle (Gornall and Kuksis, 1971) and a large proportion of the cholesterol in LDL (approx. 92\%) is extractable with ether (Evans et al., 1973). This suggests that cholesterol in egg yolk LDL is not involved in strong interactions with macromolecules and is therefore likely to be degraded more easily by COD than cholesterol present in other classes of lipoproteins which cannot be extracted with ether (Evans et al., 1973).

Specificity and Mode of Action of COD

The specificity of COD was demonstrated by comparing the rates of oxidation of several cholesterol analogues with that of cholesterol at the same molarity. COD was specific for $3 \beta$ sterols and required a double bond in the $\Delta^{5}$ or $\Delta^{4}$ position of the sterols (Richmond, 1973). Of a number of cholesterol analogues tested,

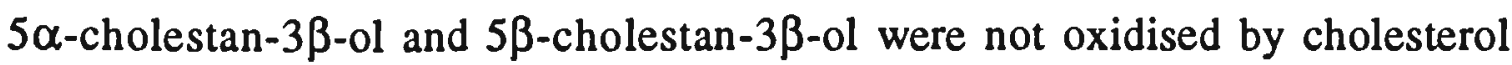
oxidase (Allain et al., 1974). 
A list of cholesterol derivatives able to interfere in the enzymatic reaction of COD (Ikawa et al., 1979) were characterised by a hydroxyl function in position $\beta$ in relation to the cyclic nuclei (Cheillan et al., 1989). Certain microorganisms also contain highly specific oxidases which oxidase one specific hydroxyl group on the third carbon in preference to another (Smith and Brooks, 1977).

In contrast, Nocardia erythropolis COD, a soil organism (Turfitt, 1944), was found to act on $5 \alpha$-cholestan- $3 \beta$-ol, such as stigmasterols and sitosterol, as well as $\Delta^{5}$-3ß-hydroxysteroids (Smith and Brooks, 1974) found in egg yolk (Tu et al., 1970). Although Nocardia erythropolis COD does not show complete specificity for cholesterol it is more specific than present colorimetric cholesterol assays and exhibits comparable precision (Flegg, 1973).

The rate of oxidation of $\Delta^{5}-3 \beta$-hydroxysteroids was also found to be influenced by side-chain length (Smith and Brooks, 1974). COD from Nocardia erythropolis showed a markedly diminished affinity for cholesterol when the side-chain was shortened (Richmond, 1973). In contrast, COD from Brevibacterium sterolicum did not show any marked change in activity as the length of the side-chain was altered (Ikawa et al., 1979).

The position and type of side-chain oxygenation also effects cholesterol oxidation (Smith and Brooks, 1974). A hydroxyl group in the 25- or 26-position of the cholesterol side-chain decreases the affinity of the enzyme for a steroid, illustrated by the difference in rates between cholesterol and 26-hydroxycholesterol (Smith and Brooks, 1974). Shortening the side chain at C-17 to just one carbon, or the 
complete removal of the side chain, results in a dramatic reduction in the rate of oxidation, though the apparent $\mathrm{Km}$ is unaffected (Smith and Brooks, 1977).

COD from Brevibacterium sterolicum catalyses the oxidation of steroids having a sterically unhindered C-3 $\beta$ hydroxyl group, but shows weak activity towards steroids with functional groups adjacent to the $3 \beta$-hydroxyl group on the steroid nucleus (Ikawa et al., 1979).

\section{Cofactors of Cholesterol Oxidase}

Like many oxidation enzymes, COD acts in association with a cofactor which ensures the transfer of electrons and hydrogen atoms from cholesterol to molecular oxygen. The oxidoreduction cofactor has been identified as covalently bound flavin adenine dinucleotide (FAD) (Kenney and Singer, 1979). FAD has also been identified as the flavin moiety of the enzyme from Brevibacterium sterolicum ATCC 21387, one mole of which was found per mole of protein (Uwajima et al., 1974).

Although, FAD is covalently bound to the enzyme, addition of exogenous FAD to the buffer solution was found to double COD activity. However, the increase in enzyme activity was not proportional to FAD concentration (Cheillan et al., 1989).

\section{Cholesterol Oxidation Byproducts}

The byproduct of cholesterol oxidation has been identified as cholesten-4-en-3one, by $\mathrm{mp}$, elemental analysis, optical rotation and ultra-violet, infra-red and 
nuclear magnetic resonance spectra (Uwajima et al., 1974). Smith and Brooks (1974) have demonstrated that cholesten-5-en-3-one, a structural isomer of cholesten-4-en-3-one, is an intermediate in this process. Cholesterol oxidase thus possesses both oxidase and isomerase activity. Investigations on substrate specificity of isomerase action using 5-cholesten-3-one, pregnen-5-ene-3,20-dione and androst-5-ene-3,17-dione, showed there was no major influence of the $\mathrm{C}-17$ side chain on the rate of isomerisation; however, in the absence of a side-chain, a large increase in the apparent $\mathrm{Km}$ was observed.

The cholesterol oxidase reaction of cholesterol to cholestenone has been described in many microorganisms including Nocardia erythropolis (Flegg, 1973; Smith and Brooks, 1974) and Nocardia rhodochrous (Cheetham et al., 1982).

\section{COD Activity and Cholesterol Assay}

The assay of COD activity is based on the conversion of cholesterol to cholestenone which has an absorption maximum of $240 \mathrm{~nm}$, owing to the conjugated carbonyl ring group in ring A (Allain et al., 1974; Flegg, 1973). Determination of the byproduct of cholesterol oxidation has been used by many investigators for the assay of cholesterol and the measurement of COD activity, as there is a stable accumulation of the product allowing analysis to be made at equilibrium (Flegg, 1973).

Auto-oxidation byproducts of cholesterol such as 25-hydroxycholesterol and 7ketocholesterol (Csallany et al., 1989) and cholesta-3,5-diene-7-one (Maerker and Unruh, 1986) have been referred to as toxic (Naber et al., 1983). The 7- 
ketocholesterol may not be detected by workers treating cholesterol with the COD enzyme, as the product has long been known to be unstable to hot aqueous alkali (Maerker and Unruh, 1986). Therefore, it would most probably be destroyed in the saponification step in routine sterol extraction. These products, however, are due to the spontaneous oxidation of cholesterol rather than enzymatic oxidation.

All previous enzymatic methods for cholesterol determination have been based on the conversion of cholesterol to cholestenone and hydrogen peroxide, which are produced in equimolar quantities. Hydrogen peroxide, produced during enzymatic reaction and serving as a basis for many electrochemical detection techniques, is an inhibitor of COD. Cholest-4-en-3-one has no effect on the enzymatic reaction (Cheillan et al., 1989).

Noma and Nakayama (1976) after studying the reaction rates at varying concentrations of cholesterol by COD from Brevibacterium and Streptomyces concluded that both enzymes were suitable for the determinations of cholesterol in hypercholesterolaemic sera.

\section{Potential Application for Cholesterol Degradation}

The potential of COD enzyme for reducing cholesterol in liquid egg yolk has been indicated by work performed on milkfat (Xiansheng et al., 1990). Milk is a complicated system, similar in many ways to egg, and was selected as a trial food system to investigate the cholesterol degrading capacity of the enzyme. When commercial milk was treated with COD isolated from Streptomyces species, up to $65 \%$ of cholesterol in milk was removed after $24 \mathrm{~h}$ incubation at low temperature 
(4-7 $\left.{ }^{\circ} \mathrm{C}\right)$ (Xiansheng et al., 1990). Other researchers have demonstrated the ability of cholesterol oxidase to degrade all of the cholesterol in a buffer system and up to $65 \%$ in milk at low temperature $\left(3-7^{\circ} \mathrm{C}\right)$, in less than $24 \mathrm{~h}$ (Uwajima et al., 1974).

The cholesterol degrading activities of cholesterol oxidases originating from four different microorganism genera, Brevibacterium, Nocardia, Pseudomonas and Streptomyces, in an egg yolk system under different environments was investigated, in order to define the optimal conditions for each enzyme. The enzyme from Nocardia is intrinsically membrane bound whereas those from Streptomyces and Brevibacterium are extracellular cholesterol oxidases (Stehbens, 1990; Cheillan et al., 1982; Smith and Brooks, 1974).

The cholesterol content of treated and untreated egg yolks were compared and the results expressed as either percent cholesterol reduction or $\mathrm{mg} / \mathrm{g}$ residual cholesterol.

\subsubsection{Results and Discussion}

\section{Effect of Time and Temperature}

The time course of cholesterol degradation resulting from each of four CODs in egg yolk at $37^{\circ} \mathrm{C}, 25^{\circ} \mathrm{C}$ and $4^{\circ} \mathrm{C}$ are shown in figures 5.1, 5.2 and 5.3. In each case, the yolk was incubated with enzyme for $72 \mathrm{~h}$.

The cholesterol degradation activity of each enzyme at $37^{\circ} \mathrm{C}$ is presented in figure 5.1 . 


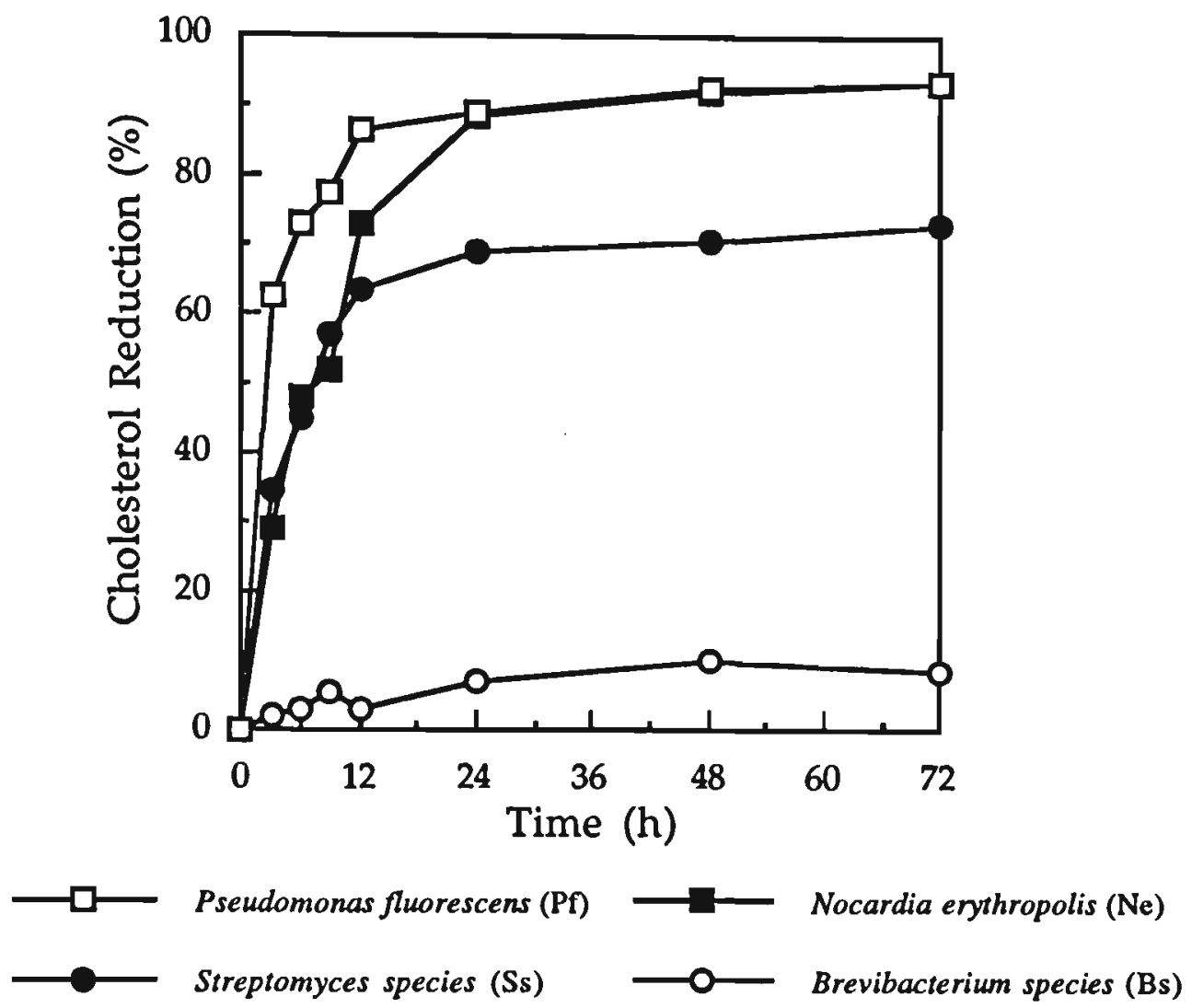

Figure 4.1 Time course of cholesterol degradation in egg yolk at $37^{\circ} \mathrm{C}$ by the four CODs investigated. Each data point is the average of triplicate samples.

At $37^{\circ} \mathrm{C}$, the Bs COD could only degrade about $8.3 \%$ of the total cholesterol after 72h of incubation while Ss COD reduced cholesterol by $72.6 \%$. Both Pf COD and Ne COD, respectively, reduced up to $93.4 \%$ of the cholesterol.

With the exception of $\mathrm{Bs} \mathrm{COD}$, cholesterol degradation at $37^{\circ} \mathrm{C}$ occurred in two stages. In the first stage, the reaction proceeded rapidly up to approximately $12 \mathrm{~h}$. In the second stage, the reaction rate became slower, and after $24 \mathrm{~h}$ remained almost unchanged. 
The performance of the $\mathrm{Pf} C O D$ at $25^{\circ} \mathrm{C}$ (figure 4.2) was similar to that at $37^{\circ} \mathrm{C}$ in terms of reaction rate and cholesterol degradation capacity.

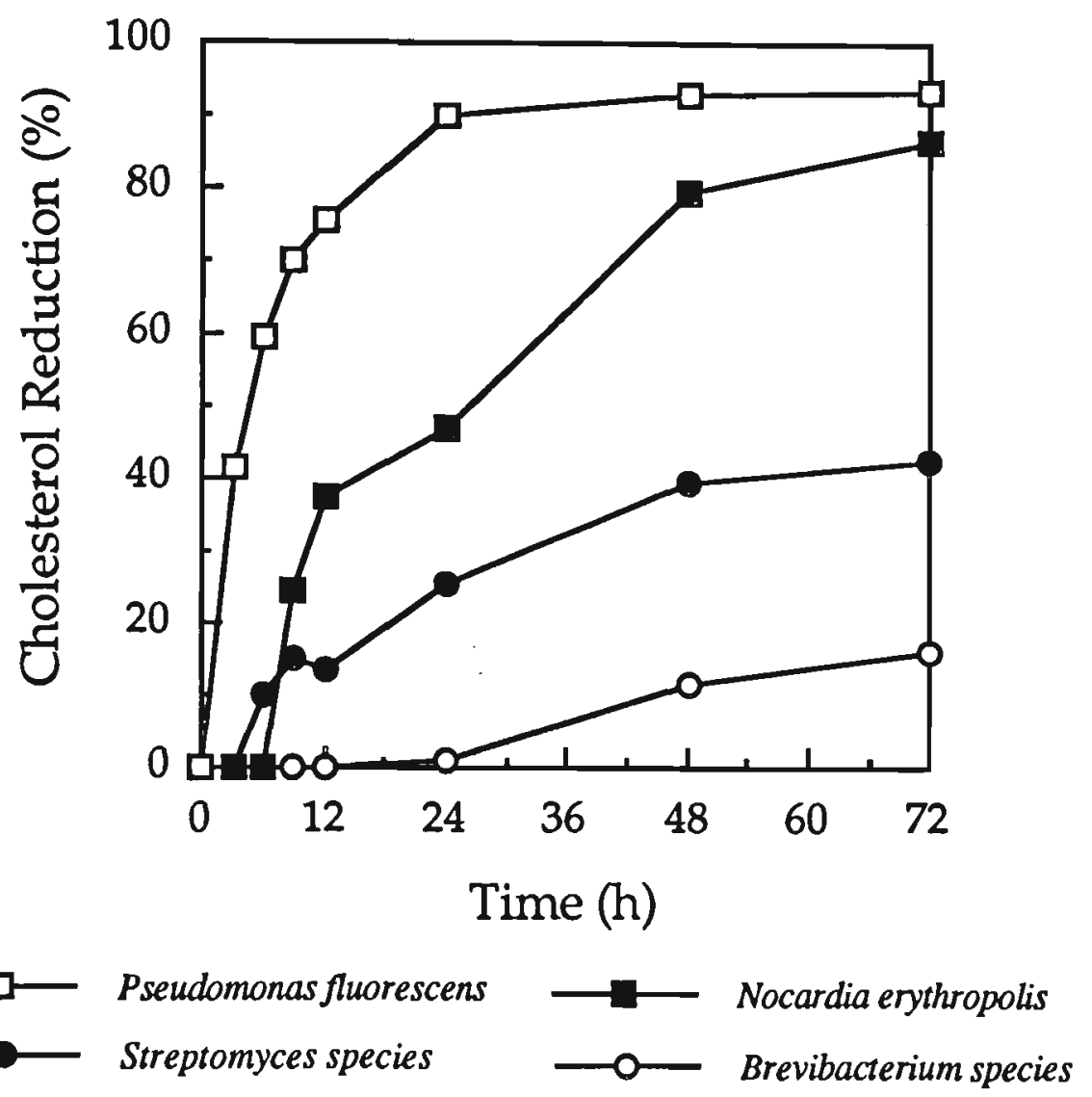

Figure 4.2 Time course of cholesterol degradation in egg yolk at $25^{\circ} \mathrm{C}$ by the four CODs investigated. Each data point is the average of triplicate samples.

The reaction of both $\mathrm{Ne}$ and Ss CODs occurred at a much lower rate for the first $48 \mathrm{~h}$ at $25^{\circ} \mathrm{C}$ compared to that at $37^{\circ} \mathrm{C}$. The cholesterol degradation capacity of both $\mathrm{Ne}$ and Ss CODs also decreased at $25^{\circ} \mathrm{C}$ with $\mathrm{Ne} \mathrm{COD}$ degrading $86.4 \%$ and Ss COD degrading $42.3 \%$ of the total cholesterol after $72 \mathrm{~h}$. Bs COD showed little reaction until $24 \mathrm{~h}$ incubation and $15.7 \%$ after $72 \mathrm{~h}$ incubation. At $4{ }^{\circ} \mathrm{C}$, all enzymes, except Pf COD, showed no or insignificant reaction even after $72 \mathrm{~h}$ of incubation (Figure 4.3). Interestingly, Pf COD could degrade approximately $30 \%$ 
cholesterol within $3 \mathrm{~h}$ and $67.2 \%$ after $48 \mathrm{~h}$, after which the cholesterol level remained almost unchanged.

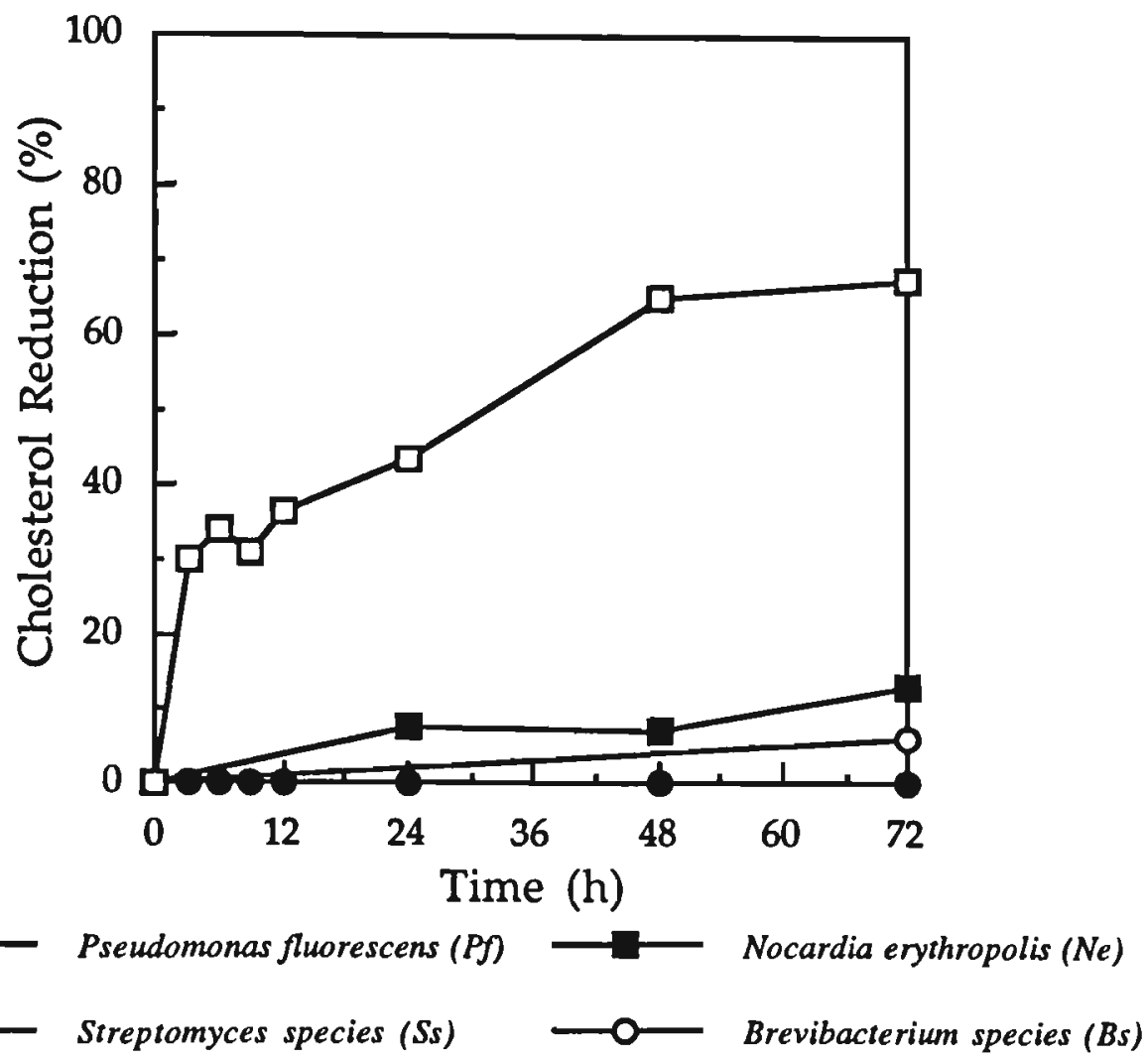

Figure 4.3 Time course of cholesterol degradation in egg yolk at $4^{\circ} \mathrm{C}$ by the four CODs investigated. Each data point is the average of triplicate samples.

In a previous study, Ss COD completely degraded free cholesterol in a buffer system within $24 \mathrm{~h}$ at $25^{\circ} \mathrm{C}$ and $37^{\circ} \mathrm{C}$ but only $60 \%$ of cholesterol at $7^{\circ} \mathrm{C}$ or $45^{\circ} \mathrm{C}$ (Uwajima et al., 1974). In milk, the Ss enzyme reduced about $35 \%$ of cholesterol after $10 \mathrm{~h}$ at $25^{\circ} \mathrm{C}$ but up to $50 \%$ at $3^{\circ} \mathrm{C}$ and $7^{\circ} \mathrm{C}$. By contrast, in the present egg yolk system, Ss COD showed high activity at $37^{\circ} \mathrm{C}(65 \%)$ and $25^{\circ} \mathrm{C}(35 \%)$ but no activity at $4^{\circ} \mathrm{C}$. This observation may reflect the effect of substrate environment on the activity of the enzyme. 
In a buffer system, Ss oxidases will readily reduce free cholesterol (100\% reduction), but the membrane bound cholesterol present in milk is more difficult to reduce (35 to 50\% reduction) (Jellema, 1980). In milk, cholesterol mainly exists in the globule surrounded by the milk fat membrane. To react with the cholesterol, the enzyme has to penetrate the membrane or the cholesterol has to be released. Ss cholesterol oxidase, may behave like some lipoproteins, with a tendency to attach to fat globules at low temperatures (Hung and Unger, 1993), thereby promoting the accessibility of cholesterol to the enzyme. This may explain why the reduction of cholesterol increased from $35 \%$ to $50 \%$ when the temperature was lowered from $35^{\circ} \mathrm{C}$ to $4^{\circ} \mathrm{C}$. At the most active range of temperature $\left(25^{\circ} \mathrm{C}\right.$ to $\left.37^{\circ} \mathrm{C}\right)$, Ss COD reacted with egg cholesterol as in a buffer system.

At $4^{\circ} \mathrm{C}$, contrary to its behaviour with milk cholesterol, the Ss enzyme failed to react with egg cholesterol, possibly due to a lack of a common bonding site for both enzyme and cholesterol, which appears to be present in the milkfat membrane at low temperature (Uwajima et al. 1979). The results show that the cholesterol degrading capacity of these enzymes follows the sequence, $\mathrm{Pf}>\mathrm{Ne}>\mathrm{Ss}>\mathrm{Bs}$ (Figures 4.1, 4.2 and 4.3). In a comparative study on CODs, Noma and Nakayama (1976) found that the degrading activity of these enzymes for pure cholesterol in a buffer system followed a reverse sequence Bs $>$ Ss $>$ Ne.

These observations appear to indicate that the activity of a COD is greatly influenced by the nature of the reaction system. For example, cholesterol oxidase isolated from Ss was found to be much more active in supercritical carbon dioxide, than in a buffer or yolk system. In supercritical carbon dioxide $\left(100 \mathrm{bar}, 35^{\circ} \mathrm{C}\right)$, 
COD from Ss demonstrated $100 \%$ cholesterol degradation within $1 \mathrm{~h}$ (Noma and Nakayama, 1976).

The ability of each of the four CODs to degrade egg yolk cholesterol, as a function of temperature, was measured by incubating each COD with egg yolk for $12 \mathrm{~h}$ at temperatures ranging from $5^{\circ} \mathrm{C}$ to $60^{\circ} \mathrm{C}$. The $12 \mathrm{~h}$ incubation period chosen was based on data presented in the initial incubation studies (figures 4.1 to 4.3 ), which showed the majority of cholesterol being degraded in the first $12 \mathrm{~h}$. The results obtained from the temperature study are presented in figure 4.4.

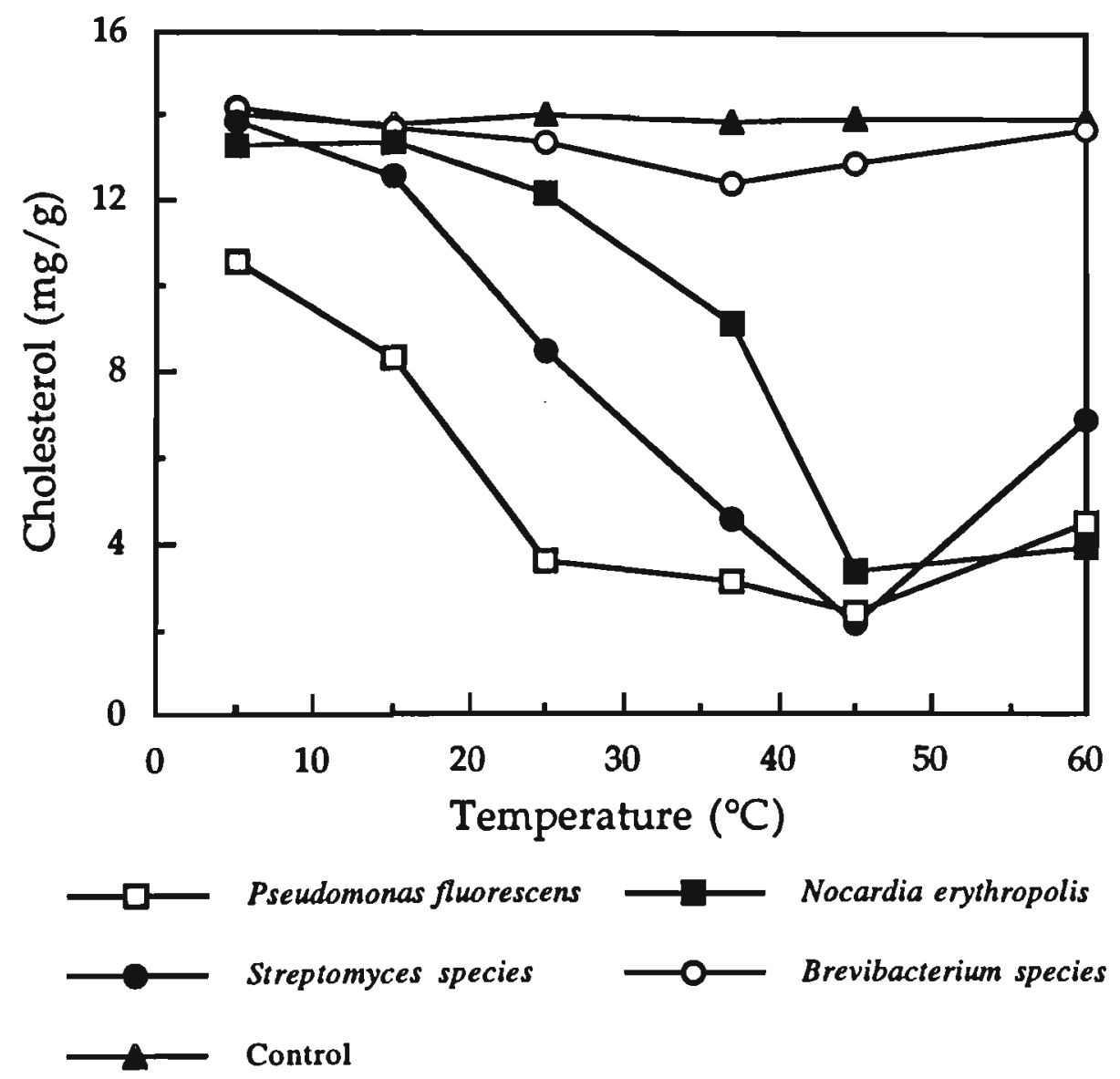

Figure 4.4 Effect of temperature on the activity of cholesterol oxidases from various CODs in egg yolk, after $12 \mathrm{~h}$. LSD (for comparing the control treatment at different temperatures) $=0.58$. LSD (for all other comparisons)=1.07. The experimental design is described in detail in Chapter 2.3.2. 
The effect of temperature on the enzyme activity after $12 \mathrm{~h}$ incubation differed greatly between enzyme types (interaction significant at $p<0.001$, Figure 4.4). An attempt was made to fit the treatment variation to linear, cubic and quadratic relationships, but none of these relationships could adequately account for all the variation among the treatment means (the lack of fit was significant, $\mathrm{p}<0.001$ ).

The most effective temperature for the $\mathrm{Pf}, \mathrm{Ne}$ and Ss CODs was around $45^{\circ} \mathrm{C}$; for Bs COD maximum activity appeared closer to $37^{\circ} \mathrm{C}$. The enzymes isolated from Pf, Ne and Ss showed good heat stability with substantial activity retained even at $60^{\circ} \mathrm{C}$. The Pf COD has an effective temperature range from $5^{\circ} \mathrm{C}$ to $60^{\circ} \mathrm{C}$, and from $5^{\circ} \mathrm{C}$ to $37^{\circ} \mathrm{C}$ it is clearly superior to the other three enzymes.

The most effective temperature/enzyme combination for maximum cholesterol degradation after $12 \mathrm{~h}$, was determined to be that by Pf COD incubated at $37^{\circ} \mathrm{C}$ ( $L S D=1.07 \mathrm{mg}$ cholesterol $/ \mathrm{g}$ ). Raising the temperature to $45^{\circ} \mathrm{C}$ did not produce a significant change in the amount of cholesterol degraded by this enzyme, and heating further to $60^{\circ} \mathrm{C}$ was no different to incubating at $25^{\circ} \mathrm{C}$ for the same period ( $\mathrm{LSD}=1.07 \mathrm{mg}$ cholesterol/mg). At the low temperature of $5^{\circ} \mathrm{C}, \mathrm{Pf} \mathrm{COD}$ was the only enzyme to achieve a cholesterol reduction (up to $28.7 \%$ ) which was significantly different to the control (LSD $=0.88 \mathrm{mg}$ cholesterol/g).

\section{Effect of Concentration}

The effect of enzyme concentration on cholesterol degradation is shown in figure 4.5. COD from Bs was not used due to the poor activity it demonstrated in the first two studies. 
Based on data presented in figure 4.4, yolk samples were incubated at the optimum temperature of $45^{\circ} \mathrm{C}$. An incubation time of $2 \mathrm{~h}$ was chosen because the data presented in figure 4.1 indicates that the reaction order followed first order kinetics for this initial period.

The effect of enzyme concentration on the degradation of egg yolk cholesterol after 2h differed significantly (interaction significant as $\mathrm{p}<0.01$, Figure 4.5 ).

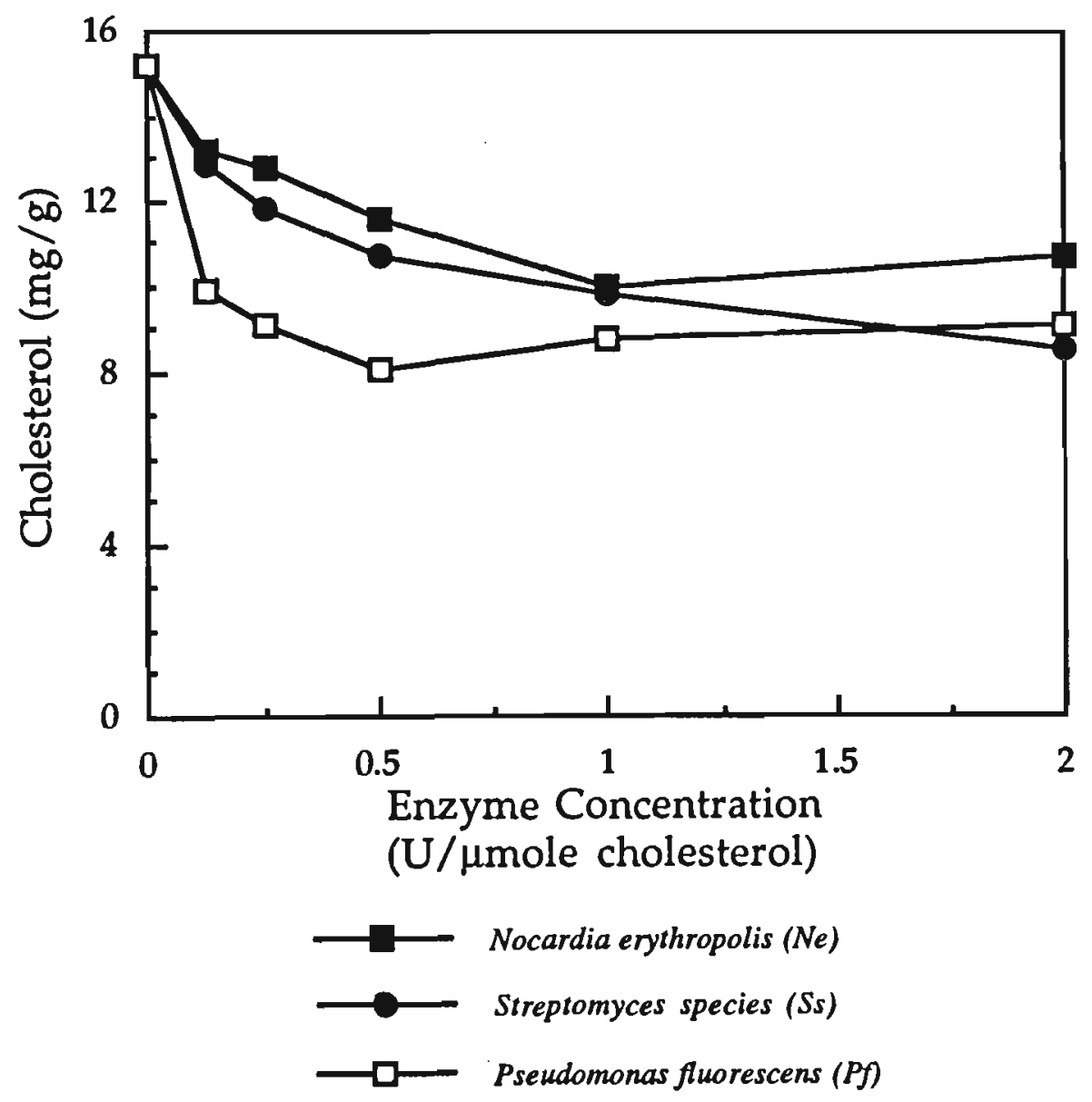

Figure 4.5 Effect of enzyme concentration on the degradation of cholesterol in egg yolk, at $45^{\circ} \mathrm{C}$ after $2 \mathrm{~h}$, by various CODs. LSD (for comparing control with non-control treatments at different concentrations) $=0.41$. LSD (for all other comparisons) $=0.47$. The experimental design is described in detail in Chapter 2.3.2. 
As in the previous study on temperature effect, an attempt was made to fit the treatment variation to linear, cubic and quadratic relationships, but none of these relationships could adequately account for all the variation among the treatment means (the lack of fit was significant, $p<0.01$ ).

The most effective concentration of Pf COD and Ne COD was around $0.5 \mathrm{U} / \mu \mathrm{M}$ cholesterol and $1 \mathrm{U} / \mu \mathrm{M}$ cholesterol, respectively. Increasing the concentration of either of these enzymes further did not produce significantly different reductions in cholesterol ( $\mathrm{LSD}=0.47 \mathrm{mg}$ cholesterol $/ \mathrm{g}$ ). Surprisingly, even at the low concentration of $0.125 \mathrm{U} / \mu$ mole cholesterol, COD from Pf degraded more than one third (up to $34.6 \%$ ) of the egg cholesterol in $2 \mathrm{~h}$. This was the only significantly different reduction between treatments seen at this concentration (LSD $=0.47 \mathrm{mg}$ cholesterol/g). Increasing the concentration of Pf COD further to 1 and $2 \mathrm{U} / \mu \mathrm{M}$ did not produce a reduction in cholesterol that was significantly different (LSD $=0.47 \mathrm{mg}$ cholesterol $/ \mathrm{g}$ ).

Cholesterol degradation was significantly different to that of the control at all concentrations and enzyme types ( $\mathrm{LSD}=0.41 \mathrm{mg}$ cholesterol $/ \mathrm{g}$ ). The most effective enzyme type/concentration combination was that of Pf COD at $0.5 \mathrm{U} / \mu \mathrm{M}$.

\section{Cholesterol Degradation Products}

A number of COD products have been detected in various foods, including spraydried egg yolk powder (Larsen and Froning, 1981). The adverse health effects of these oxidation products are still a topic for debate. 
In this study, the degradation products of egg cholesterol were examined by TLC and confirmed by MSD. The only steroid-like component detected was 4-

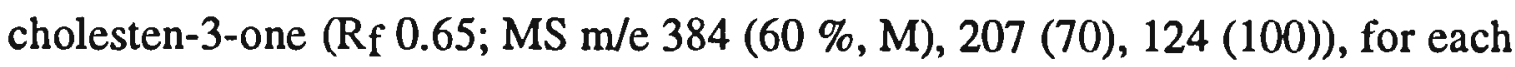
of the four enzymes. The 4-cholesten-3-one has been described as the first degradation product of cholesterol oxidase catalysis in species of Nocardia, Brevibacterium, Pseudomonas and Streptomyces by many investigators (Aihara et al., 1988a; Cheetham et al., 1982; Flegg, 1973; Smith and Brooks, 1974; Tomioka et al., 1976; Uwajima et al., 1974).

In milk inoculated with Ss COD, 4-cholesten-3-one was formed but degraded further after $25 \mathrm{~h}$ of incubation at both low $\left(3^{\circ} \mathrm{C}\right.$ to $\left.7^{\circ} \mathrm{C}\right)$ and high temperature $\left(25^{\circ} \mathrm{C}\right.$ to $45^{\circ} \mathrm{C}$ ) (Aihara et al., 1988a). Aihara et al. (1988a) also found this compound in egg yolk inoculated with $R$. equi No. 23, a COD producing microorganism, but it was converted rapidly into non-steroid components. 


\section{CHAPTER 5}

Microbial Degradation of Egg and Milk Cholesterol 


\subsection{Introduction}

Cholesterol in milk and egg yolk can be degraded by isolated cholesterol oxidase enzymes (Xiansheng et al., 1990), and by some intact microorganisms (Aihara $e t$ al., 1988). Xiansheng et al. (1990) has demonstrated the ability of cholesterol oxidase isolated from Streptomyces species to completely degrade cholesterol in a buffer system at $25^{\circ} \mathrm{C}$ and $37^{\circ} \mathrm{C}$, and up to $65 \%$ of the cholesterol in milk at lower temperatures $\left(3-7^{\circ} \mathrm{C}\right)$, in less than $24 \mathrm{~h}$. Work described in Chapter 5 of this thesis indicates that up to $93 \%$ of egg yolk cholesterol is degraded by cholesterol oxidase from Pseudomonas fluorescens and Streptomyces species after incubation for $3 \mathrm{~d}$ at $37^{\circ} \mathrm{C}$ and up to $64.9 \%$ with isolated COD from Pseudomonas fluorescens after incubation for $2 \mathrm{~d}$ at $4^{\circ} \mathrm{C}$.

The degradation of egg yolk cholesterol by Rhodococcus equi No. 23, a microorganism isolated from butter, has been described by Aihara et al. (1988a); an extracellular, membrane-bound form of the COD enzyme was identified in different strains of Rhodococcus with the highest levels of activity being produced by strain No. 23. This information was suggested as important for the industrial preparation of COD from culture filtrate, which can be used for the specific estimation of cholesterol in blood serum (Aihara et al., 1986).

\subsubsection{Equi Factor}

The extracellular enzyme system secreted by $R$. equi No. 23 has been reported to contain an additional enzyme, phospholipase $\mathrm{C}$, which encourages the degradation of cholesterol by cholesterol oxidase through decomposition of components in the 
LDL-surface layer in egg yolk resulting in greater substrate availability. This cholesterol degrading system has been referred to as the "equi factor" (Aihara $e t$ al., 1988b, Prescott et al., 1982).

Moore et al. (1977) reported that digestion of LDL with phospholipase C increased the amount of lipid extracted by ether from $19 \%$ to $74 \%$. The $10 \%$ of cholesterol that could be degraded without the digestion of phospholipid in the LDL-surface layer might indicate the presence of a small proportion of cholesterol which bound weakly with phospholipid, or existed in a more external position, than the major proportion of cholesterol.

Equi factor has been shown to be produced by 173 strains of serologically verified Corynebacterium equi, more commonly known as R. equi (Prescott et al., 1982). However, the ability of $C$. equi to produce equi factor(s) should not be used as verification of the strain, since other organisms may produce similar effects, or there also may be a small number of $C$. equi strains which do not produce equi factor(s) (Prescott et al., 1982).

Unfortunately, LDL plays an important role in the functional properties of egg yolk, such as emulsification and gelation, and therefore the lowering of these properties through LDL degradation by the enzymatic reaction is disadvantageous for the industrial uses of such treated egg yolk (Aihara et al., 1988b). Further studies, for example using phospholipase $\mathrm{C}$ inhibitors, are needed to produce egg yolk LDL which contains less cholesterol than native LDL, but still allows the yolk to retain its functional properties (Aihara et al., 1988b). 


\subsubsection{Cell Activity}

On comparison with strains of the genera Nocardia, Brevibacterium and Microbacterium the rate of cholesterol degradation was much higher in strains of Rhodococcus. Some strains of Rhodococcus erythropolis can decompose between $90 \%$ and $100 \%$ of the cholesterol in various animal fats. The cholesterol content in chicken fat was reduced by $63 \%$ after $24 \mathrm{~h}$ and by $97 \%$ after $72 \mathrm{~h}$ of treatment with this organism (Watanabe et al., 1986).

Johnson and Somkuti (1990) have reported that sonicated extracts of $R$. equi can degrade $40 \%$ of cholesterol in egg yolk and up to $2.4 \%$ in a milk cream preparation; the sonicated cell preparations were more effective in degrading free cholesterol than cholesterol in complex food systems such as milk cream and egg yolk.

The differences observed between Rhodococcus strains to degrade free cholesterol and cholesterol in egg yolk or milk cream could be due to (1) the presence of additional enzyme systems (esterase, phospholipase) contributing to the general destabilisation of cholesterol-binding structures in egg yolk and milk cream, thus resulting in greater substrate availability, and (2) the limited accessibility of cholesterol in egg yolk and milk cream to the enzymes of the Rhodococcus dissimilation system (Johnson and Somkuti, 1990).

The activity of the cells has also been shown to be somewhat dependent on the culture growth medium. $R$. equi cells pre-incubated without cholesterol exhibited only $58 \%$ of the activity of cells pre-incubated with cholesterol, with pre-incubated 
cells degrading nearly all of the cholesterol in a reaction system within $2 \mathrm{~h}$ (Johnson and Somkuti, 1990).

\subsubsection{Steroid Byproducts}

The product of cholesterol dissimilation with Rhodococcus strains was found to be cholest-4-en-3-one, the first product of cholesterol degradation, confirming the presence of an oxidative pathway in this organism (Johnson and Somkuti, 1990). Cholestenone has also been reported as the sole steroidal product of cholesterol oxidation by Rhodococcus strains (Richmond, 1973; Aihara et al., 1986). Steroldecomposing microorganisms that accumulate only cholestenone as the degraded product, include bacteria, actinomycetes, moulds and yeasts (Watanabe et al., 1989; Arima et al., 1969)

Compared with the mammalian system where the A-ring is kept intact until cleavage of the side-chain, converting cholesterol into pregnenolone, is accomplished, microbial decomposition occurs at first in the A-ring, including dehydrogenation, isomerisation of a double bond, and $\Delta^{1}$-dehydrogenation. Decomposition proceeds by the following pathway (Arima, 1969):

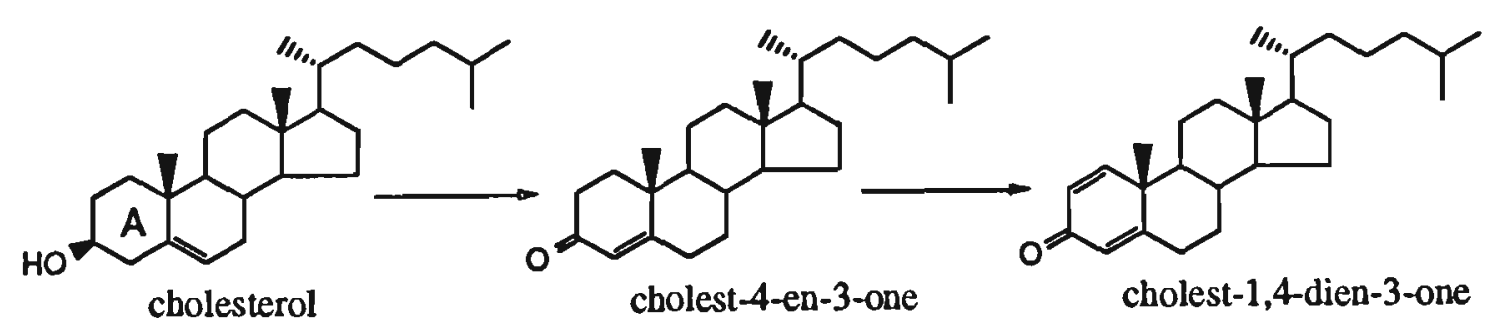




\subsubsection{Non-Steroid Byproducts}

Some microorganisms which can degrade cholesterol without accumulating steroid intermediates have also been isolated (Aihara et al., 1986, 1988; Noma and Nakayama, 1976, Johnson and Somkuti, 1990). When egg yolk was inoculated with Rhodococcus equi, up to $70 \%$ of cholesterol was degraded with almost no accumulation of steroid intermediates (Aihara et al., 1988a).

Aihara et al. (1986) found that the amounts of cholest-4-en-3-one as an intermediate from cholesterol to lower degradation products differed among strains. Up to approximately $24 \mathrm{~h}$, cholesterol in the culture medium had been decomposed quantitatively to nearly zero at a high rate in the three strains, and 4cholesten-3-one was formed as cholesterol decreased.

The virtual absence of other degradation products such as androst-1,4-dien-3,17dione and androst-4-en-3,17-dione suggests that the cholest-4-en-3-one formed was quickly converted into non-steroid intermediates. This conversion rate with almost no accumulation of steroid intermediates in culture medium was previously demonstrated for the degradation of cholesterol by Pseudomonas species NCIB 10590 (Owen et al., 1983).

The cholesterol degrading capacity of four strains of the genera Rhodococcus were investigated to determine their cholesterol degrading capacities in egg yolk and milk. 


\subsection{Results and Discussion}

R. equi No. 23 is one of a number of organisms that utilise cholesterol as sole carbon and energy sources (Watanabe et al., 1986). Some of these cholesterol degrading organisms are highly effective at utilising free cholesterol but much less so with cholesterol in complex food systems such as egg yolk and milk cream (Johnson and Somkuti, 1990).

Strains of $R$. equi No. 23 visible under oil immersion and high resolution (X 1000) all showed gram positive staining (purple colour). In all strains the morphogenetic cycle is initiated with the coccus or short rod stage (Goodfellow, 1984) (Figure 5.1).
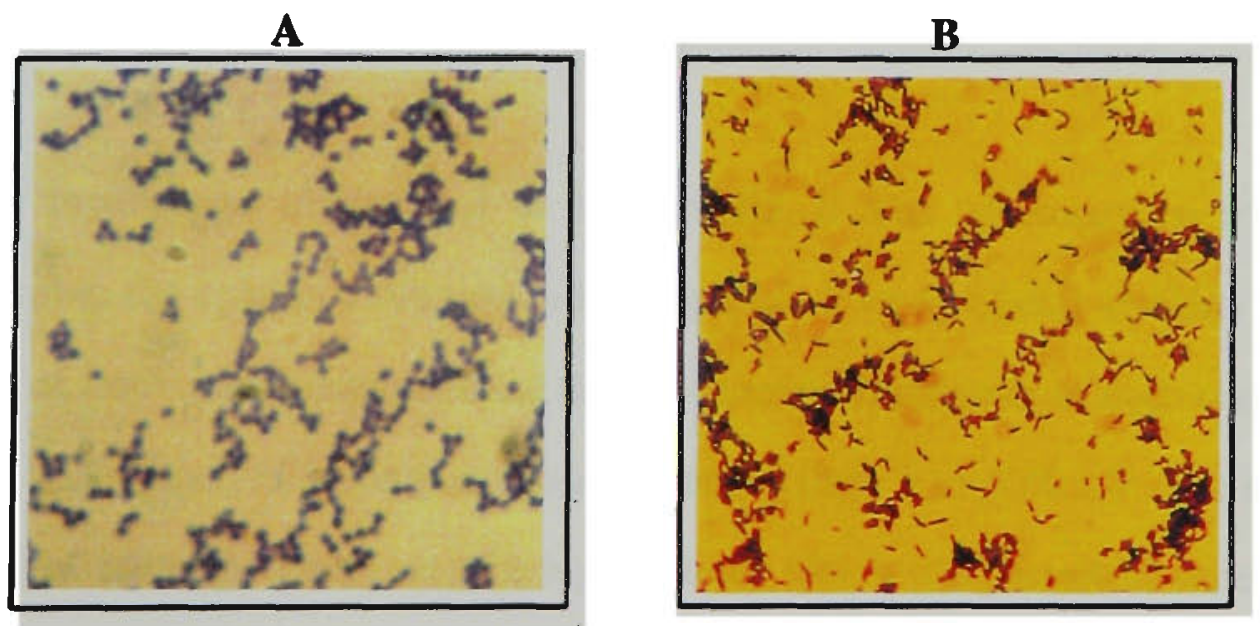

Figure 5.1 Photographs of (A) R. equi No. 23 cells as cocci and (B) $R$. equi No. 23 cells as rods under oil immersion (X 1000).

Cultures, $R$. equi No.23, $R$. erythropolis No. 3, $R$. species No.13 and $R$. species No.15 were grown in nutrient broth (Figure 5.2) and harvested at approximately $12,45,48$ and 50h, respectively, for cholesterol degradation studies. 


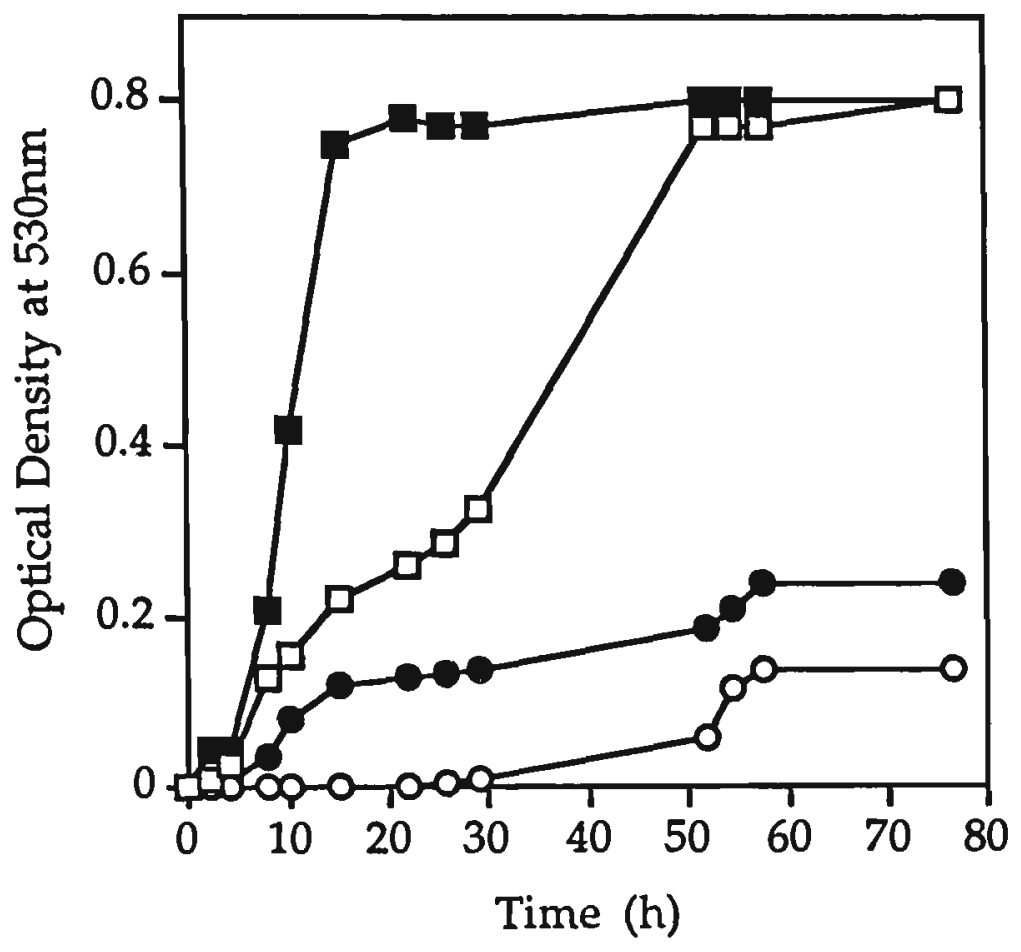

$\begin{array}{llll}- \text { R. equi No. } 23 & - & \text { R. species No. } 15 \\ \square & \text { R. erythropolis No. } 3 \longrightarrow & \text { R. species No. } 13\end{array}$

Figure 5.2 Turbidity measurements at $530 \mathrm{~nm}$ of $10 \mathrm{~mL}$ cultures inoculated with $250 \mu \mathrm{L}$ stock culture over time with mechanical shaking, at $30^{\circ} \mathrm{C}$

In order to disperse cholesterol in agar media, it was necessary to add the cholesterol to the agar as a solution in isopropanol. A study of cell viability in the presence of isopropanol demonstrated that the number of colonies of each organism declined as the level of isopropanol was increased from 0 to $50 \% \mathrm{v} / \mathrm{v}$. The results showed that $5 \%$ isopropanol was the highest level which still permitted acceptable cell viability. 


\subsubsection{Detection of Cholesterol Degradation Activity in Agar Media}

Halo formation around colonies grown on agar media containing added cholesterol was an indication of the conversion of cholesterol to cholest-4-en-3-one, the first product of cholesterol degradation, by the COD enzyme secreted by Rhodococcus organisms (Aihara et al., 1986, Watanabe et al., 1989).

Figure 5.3 shows the difference between the four strains in halo formation around colonies on the agar medium with and without added cholesterol. Activity was detected around colonies of $R$. equi No. 23 grown on agar medium with added cholesterol evident by the presence of a halo, but not around colonies grown on the cholesterol-free medium. A halo was not detected around colonies for the other three Rhodococcus strains on either media indicating little or no activity present. A higher production of cholesterol oxidase, both extracellular and membrane-bound, was found to be produced by $R$. equi No. 23 when compared to other strains of Rhodococci confirming an oxidative pathway in this organism (Aihara et al., 1986, Watanabe et al., 1989).

To examine the effectiveness of $R$. equi No. 23 in a complex food system, the organism was grown on agar containing egg yolk. A halo formation was observed around colonies of $R$. equi No. 23 grown on this medium (Figure 5.4). The percentage of remaining cholesterol in the vicinity of colonies of $R$. equi grown on yolk-containing media were determined. The remaining cholesterol in vicinity of the colony was $0.05 \mathrm{mg} / \mathrm{g}$ (halo zone 1 ) and $1.02 \mathrm{mg} / \mathrm{g}$ (halo zone 2 ) corresponding to a cholesterol reduction of $95 \%$ and $75 \%$, respectively. 

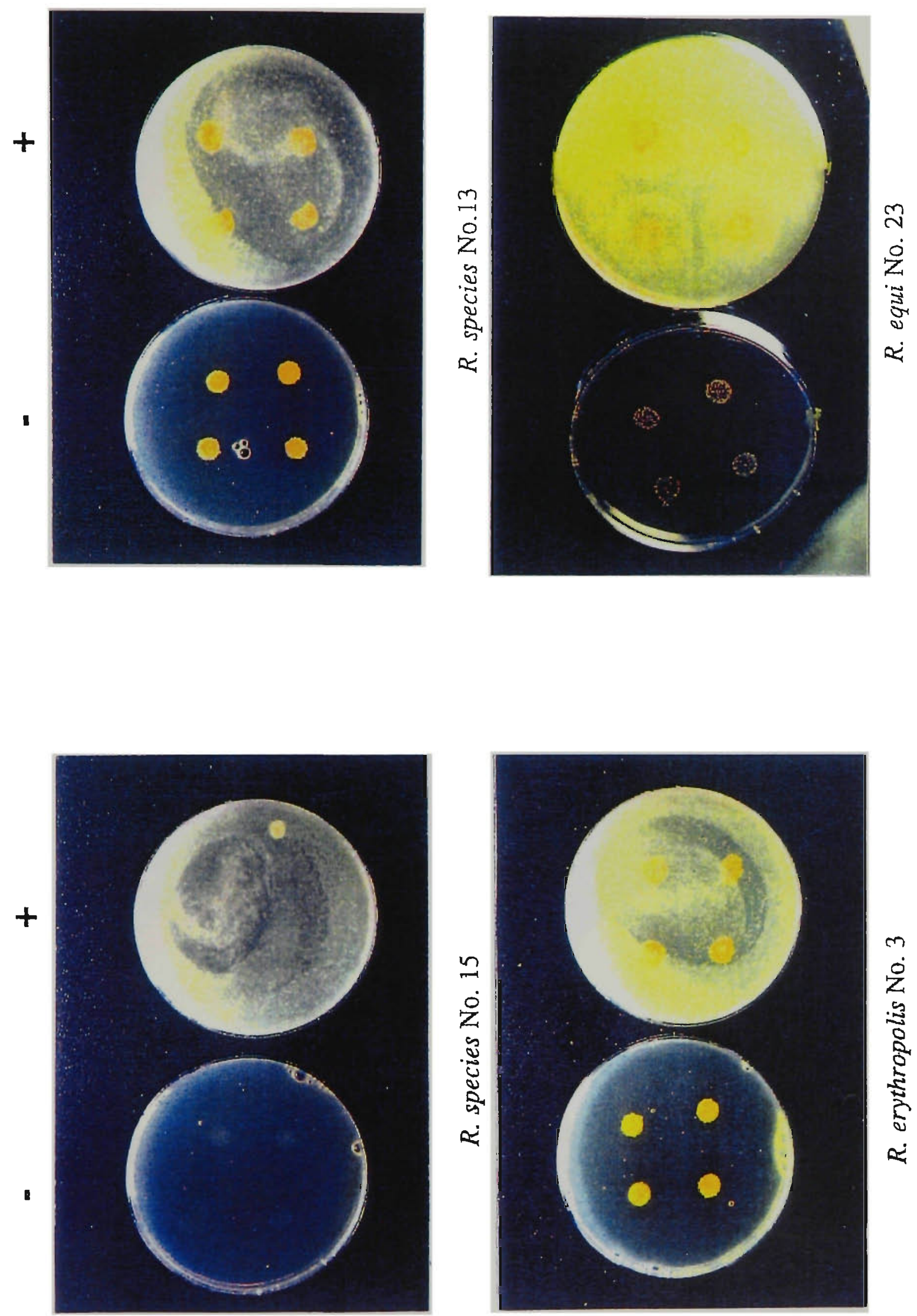

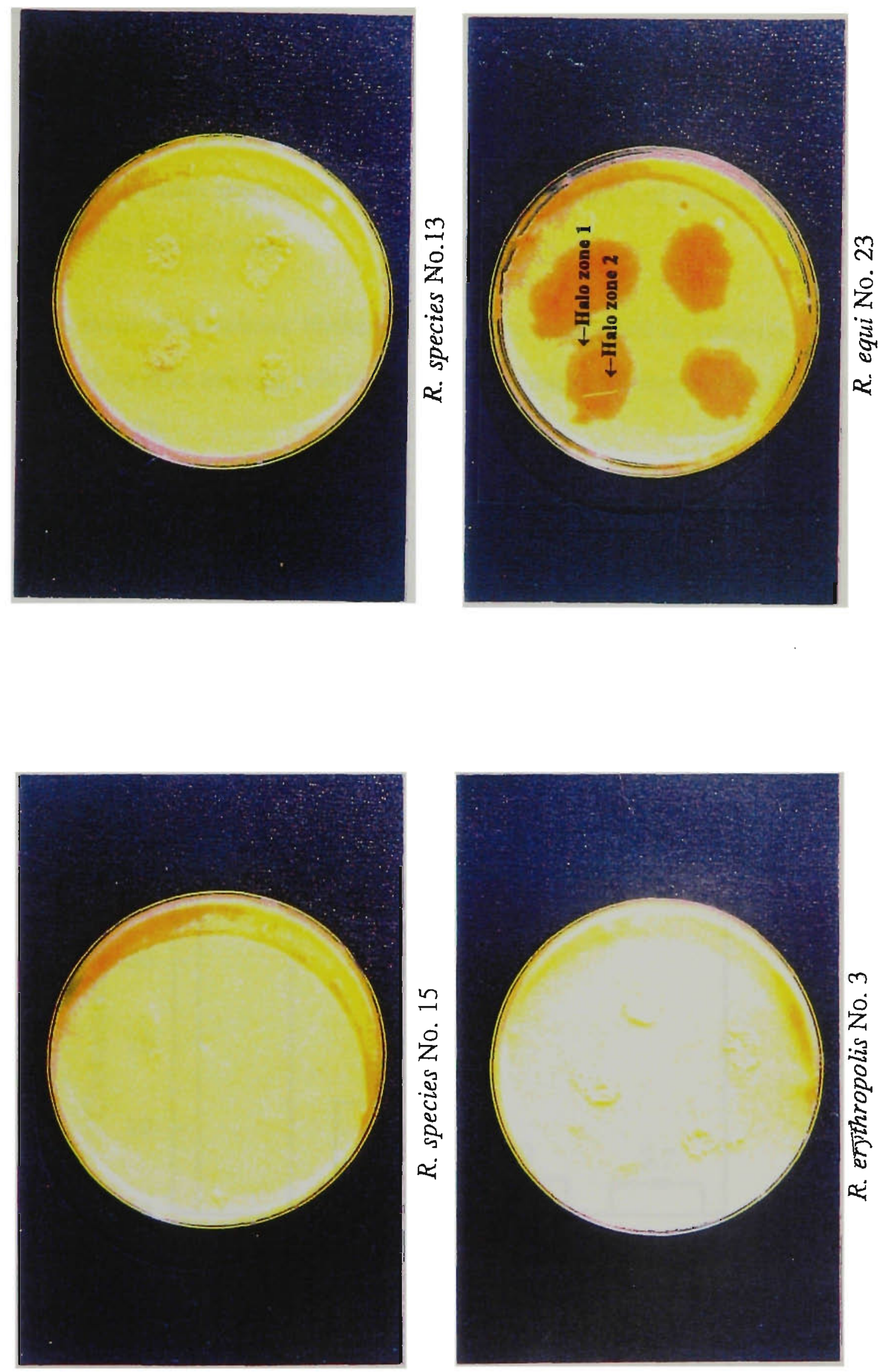
The degradation product, cholest-4-en-3-one, was detected by gas chromatography-mass spectrometry, confirming that cholesterol degradation had occurred within the halo zone.

Based on these observations further studies were conducted in complex food systems such as milk and egg yolk to examine the cholesterol degradation activity of $R$. equi No. 23.

\subsubsection{Cholesterol Degradation in Milk}

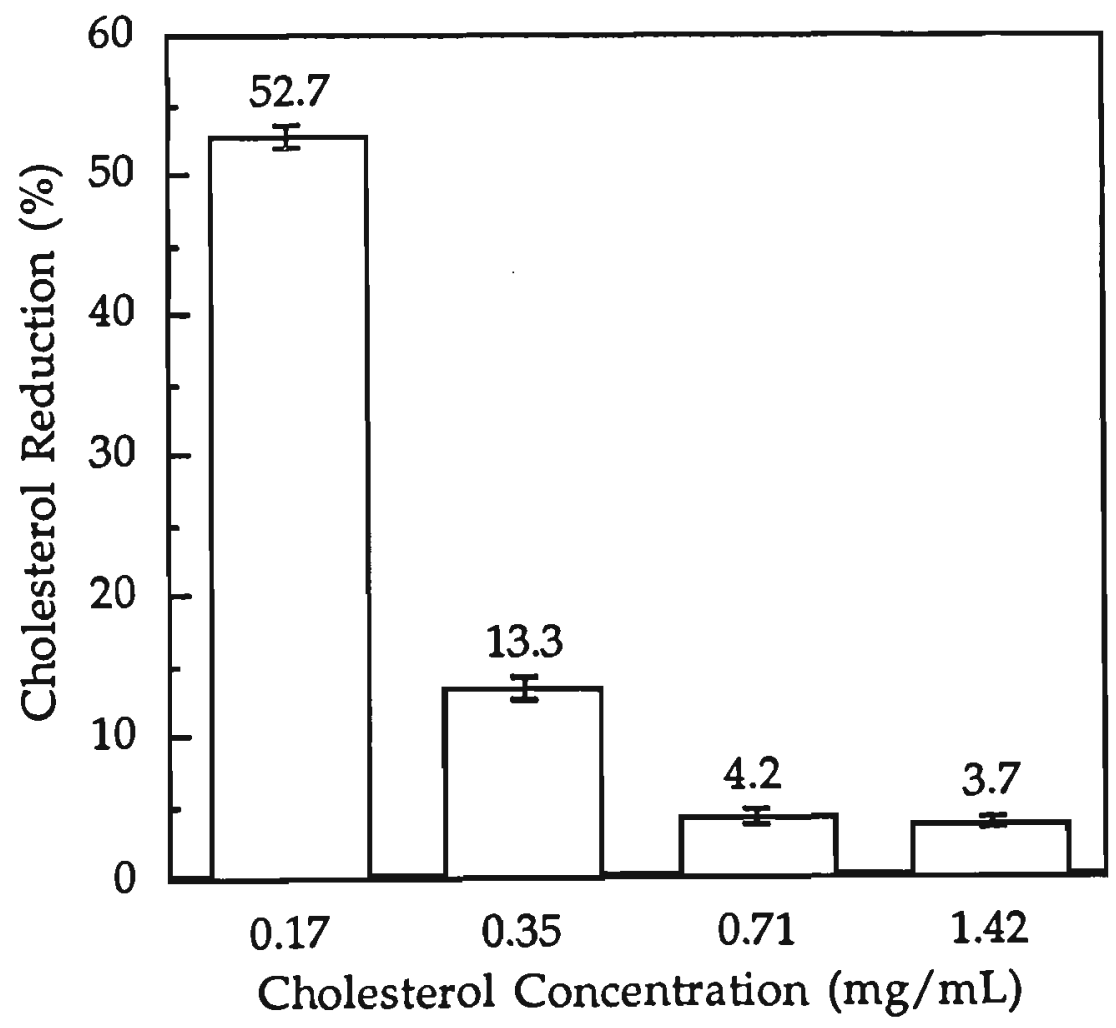

Figure 5.5 Cholesterol reduction in whole milk and in milk diluted, 1 in 2, 1 in 4, and 1 in 8, following incubation with $R$. equi No. 23 at $37^{\circ} \mathrm{C}$ for $7 \mathrm{~d}$. 
Most strains of Rhodococcus have been reported to grow between $15^{\circ} \mathrm{C}$ and $40^{\circ} \mathrm{C}$ (Goodfellow, 1984). The cholesterol degrading capacity of $R$. equi No. 23 was determined in a milk system at $37^{\circ} \mathrm{C}$ for $7 \mathrm{~d}$ (Figure 5.5). The results show that the percentage of cholesterol reduced decreased as the cholesterol substrate concentration increased from 0.17 to $1.42 \mathrm{mg} / \mathrm{mL}$.

Around $4 \%$ of cholesterol was degraded by $R$. equi No. 23 in whole milk $(1.42 \mathrm{mg} / \mathrm{mL}$ cholesterol) and in milk diluted 1 in $2(0.71 \mathrm{mg} / \mathrm{mL}$ cholesterol). Although the magnitude of degradation in both milks was small, the reduction in cholesterol was statistically significant in both the undiluted milk $(\mathrm{p}<0.01)$ and milk diluted 1 in 2 ( $p<0.05)$. When milk was diluted 1 in $4(0.35 \mathrm{mg} / \mathrm{mL}$ cholesterol) and 1 in $8(0.17 \mathrm{mg} / \mathrm{mL}$ cholesterol) cholesterol degradation increased to $13 \%$ $(\mathrm{p}<0.05)$ and $52 \%(\mathrm{p}<0.01)$, respectively.

\subsubsection{Effect of Culture Growth Medium on the Cholesterol Degrading Activity of} Cells in Milk and Egg Yolk

The conditions under which cells are grown may affect their cholesterol degrading capacity. Aihara et al. (1988b) recommended that $R$. equi No. 23 should be pregrown in cholesterol-containing medium to enhance the cholesterol reducing activity. Johnson and Somkuti (1990) also reported that cells grown in a cholesterol substrate have been induced to produce the cholesterol degrading enzymes required for cholesterol dissimilation. 


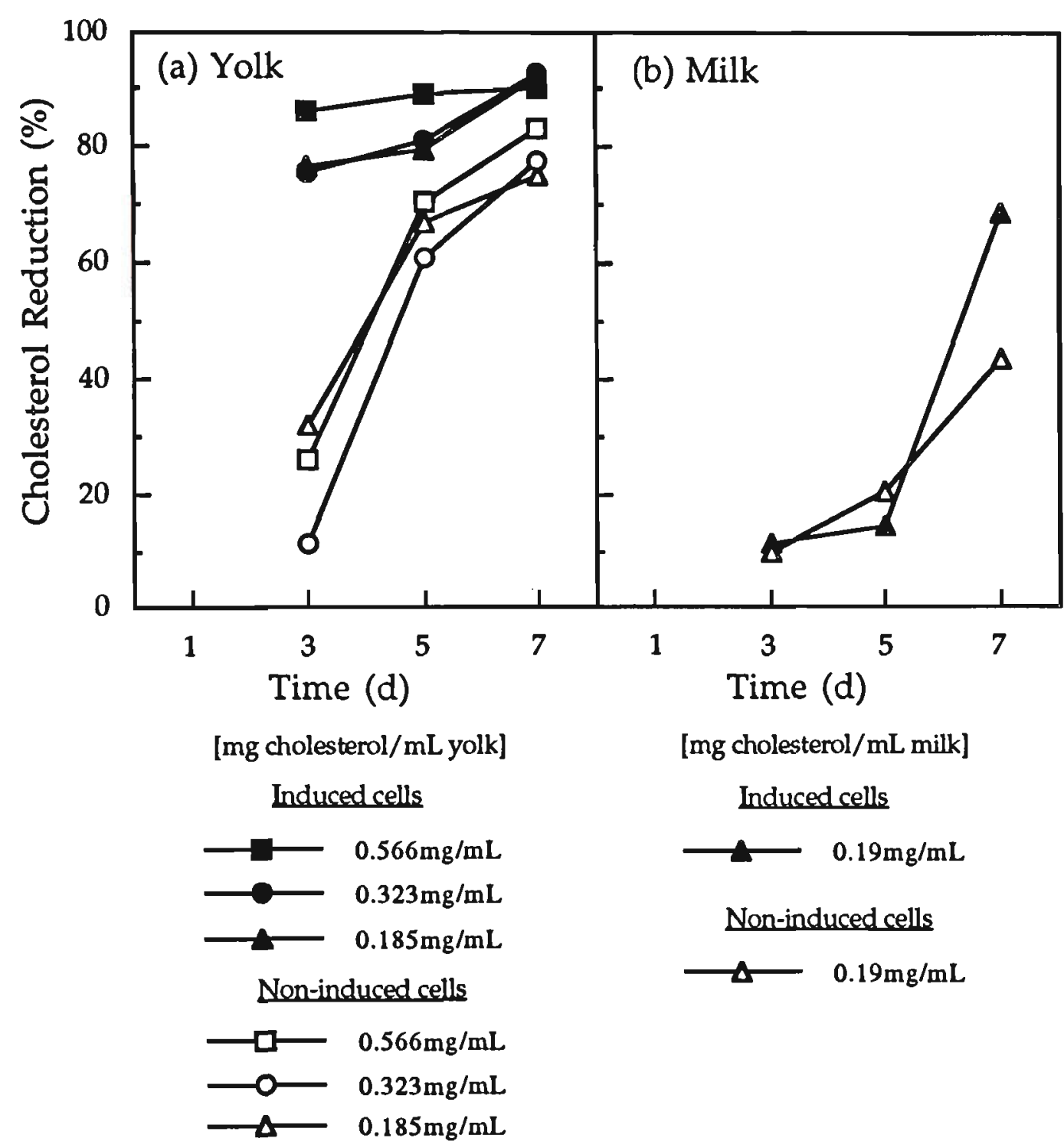

Figure 5.6 Time course of cholesterol degradation in (a) yolk and (b) milk, by induced and noninduced cells of $R$. equi No. 23

To examine the potential effect of growth conditions on the induction of cholesterol degradation activity, cells of $R$. equi No. 23 previously grown in the presence (induced) and absence of cholesterol (non-induced) were grown for 3,5 and 7d at $37^{\circ} \mathrm{C}$ in milk and egg yolk. The cholesterol degrading capacity of these cells in a yolk system at $37^{\circ} \mathrm{C}$ for up to $7 \mathrm{~d}$ is shown in figure 5.6(a). Induced cells of $R$. 
equi No. 23 showed a higher level of cholesterol degrading activity in egg yolk than non-induced cells.

For each of the three yolk cholesterol concentrations investigated, more than $70 \%$ of cholesterol was degraded after $3 \mathrm{~d}$ incubation with induced cells; the same level of degradation took $7 \mathrm{~d}$ to achieve with non-induced cells. A similar result was obtained in a free cholesterol system (Johnson and Somkuti, 1990). The faster rate of cholesterol reduction by induced cells of $R$. equi No. 23 is likely to be due to a shortened lag phase in cell growth.

Cholesterol degradation was almost complete after $7 \mathrm{~d}$ incubation with induced cells of $R$. equi No. 23 at the three cholesterol concentrations investigated, $0.57,0.32$ and $0.19 \mathrm{mg} / \mathrm{mL}(90,92$ and $92 \%$, respectively) (Figure 5.6(a)); there was no significant difference $(\mathrm{p}>0.05)$ between the amounts of cholesterol degraded after $7 \mathrm{~d}$ incubation for each of the cholesterol concentrations. Similarly, the amounts of cholesterol degraded by non-induced cells of $R$. equi No. 23 at 7 d, for each cholesterol level, did not differ significantly $(p>0.05)$.

The cholesterol degrading capacity of non-induced and induced cells of $R$. equi No. 23 in a milk system at $37^{\circ} \mathrm{C}$ after $7 \mathrm{~d}$ is shown in figure. 5.6(b). A greater reduction in cholesterol was observed in milk inoculated with induced cells of $R$. equi compared to milk inoculated with non-induced cells, $68 \%$ and $43 \%$, respectively. 


\subsubsection{A Comparison of Milk and Egg Yolk Cholesterol Degradation}

The cholesterol degrading system of $R$ equi No. 23 was effective at degrading both milk and yolk cholesterol. In milk, cholesterol degradation was greater at the lower levels of cholesterol investigated. However, it was only at very low milk cholesterol concentrations $(0.17 \mathrm{mg} / \mathrm{mL})$ that a substantial amount of cholesterol was degraded ( $>50 \%$ after $7 \mathrm{~d}$ ). This was in sharp contrast to egg yolk where a significant amount of cholesterol was degraded at levels as high as $0.57 \mathrm{mg} / \mathrm{mL}$ $(>80 \%$ after $7 \mathrm{~d})$.

One interpretation of these results is that the COD enzyme had become saturated at a lower substrate concentration in milk than in egg yolk. All enzymes show the saturation effect, but vary widely with respect to the substrate concentration required to produce the effect (Lehninger, 1975a). Alternatively, the cholesterol in milk may have been less accessible to the cholesterol dissimilating system of Rhodococci than cholesterol in egg yolk. A similar hypothesis was proposed by Johnson and Somkuti (1990) in their investigations with yolk and milk cream.

\subsubsection{Products of Cholesterol Degradation in Egg Yolk}

The changes in the degradation products of yolk cholesterol were followed by TLC. Though diminishing amounts of cholesterol $\left(R_{f} 0.47\right)$ were observed as the cholesterol level was lowered, degradation products were not detected in the media containing $0.19 \mathrm{mg} / \mathrm{mL}$ and $0.32 \mathrm{mg} / \mathrm{mL}$ cholesterol. These results may suggest that if any steroids were formed, they were rapidly converted to non-steroid products 
without accumulation of steroid intermediates. However, minute amounts of the degradation product cholest-4-en-3-one were detected by GC-MS in media containing $0.56 \mathrm{mg} / \mathrm{mL}$ yolk cholesterol only. Cholest-4-en-3-one was characterised by the molecular ion at $\mathrm{m} / \mathrm{e} 384(46 \%, \mathrm{M})$ and fragment ions at 229 (57) and 124 (100). The absence of steroid intermediates was also reported by Aihara et al. $(1986,1988)$ in their investigation of cholesterol degradation by $R$. equi No. 23 in egg yolk and a pure cholesterol system. 


\title{
CHAPTER 6
}

\author{
Conclusions
}




\subsection{Development of a Rapid Cholesterol Assay}

Foods in which cholesterol occurs include food products with different and complex matrixes. This presents problems in determining accurate values for cholesterol. A modified method of Kovacs (1990) using solvent extraction and gas chromatographic determination was developed and used to measure cholesterol in eggs. The results obtained in this study indicated that there was less variation and better repeatability among replicate extractions with the modified technique. The modified procedure was superior to the original method since both higher cholesterol values and higher cholesterol recoveries were obtained, suggesting more effective cholesterol extraction. The modified procedure requires less solvent and less time than the original procedure.

Increased public awareness to the effect of cholesterol on CHD and the relationship of blood cholesterol to the diet has made it necessary to have a rapid, reliable and accurate method to satisfy nutritional labelling demands (Al-Hasani et al., 1990). The method employed appears to provide a greater specificity and accuracy in analysing food with different and complex matrixes.

\subsection{Cholesterol Content in Foods}

The effectiveness with which cholesterol was extracted from egg yolk by the modified extraction method was also effective in extracting cholesterol from more complex food matrixes. The modified extraction procedure was used to measure the cholesterol levels in a number of foods. The accuracy of the modified procedure to measure cholesterol in other food matrixes, however, was not 
demonstrated as reproducibility and recovery studies were not undertaken. The modified procedure successfully demonstrated the ability to extract cholesterol from non-egg based foods as well as egg-based foods.

\subsection{Cholesterol Degrading Ability of COD in Egg Yolk}

The cholesterol degrading capacity of four COD enzymes has been investigated in egg yolk, a complex food system. All enzymes catalysed the transformation of cholesterol to cholest-4-en-3-one. More than 50\% of egg cholesterol could be reduced by three CODs (Ne, Pf and Ss), within 24 hours. The Pf COD was effective over a wider temperature range $\left(4-60^{\circ} \mathrm{C}\right)$ than the other enzymes studied. The low concentration of COD from Pf required to achieve significant reduction of yolk cholesterol, coupled with the ability of this enzyme to degrade cholesterol at $4^{\circ} \mathrm{C}$, suggests that this enzyme may provide an economical and practical approach for lowering the cholesterol level in egg yolk on a commercial scale.

\subsection{Cholesterol Degrading Ability of Four Microorganisms in Egg Yolk and Milk}

The cholesterol degrading ability of four microorganisms was investigated in both whole milk and egg yolk. Of the organisms studied $R$. equi was the only one effective in degrading cholesterol; this was evident by halo formation about colonies of $R$. equi grown on cholesterol-containing agar medium. The investigations revealed that $R$. equi was effective in reducing cholesterol in yolk but much less so in milk. In egg yolk, a substantial proportion of cholesterol was degraded $(>70 \%)$ after $7 \mathrm{~d}$ at each of the three cholesterol levels studied. After the 
same period of incubation, milk cholesterol at a level comparable to the lowest concentration of yolk cholesterol $(0.19 \mathrm{mg} / \mathrm{mL})$, was only degraded by $44 \%$. In milk, appreciable cholesterol reduction was only observed at the lowest level of cholesterol studied; at the higher levels $(0.35-1.42 \mathrm{mg} / \mathrm{mL})$ cholesterol reduction was less than $15 \%$.

The culture growth medium of the $R$. equi inoculum had a marked affect on the magnitude of cholesterol degradation in both food matrixes. Cells harvested from cultures pre-grown in the presence of cholesterol, degraded more cholesterol in both milk and egg yolk than cells pre-grown in the absence of cholesterol. After 3d incubation, induced cells in egg yolk degraded up to 7 times the amount of cholesterol at the lower cholesterol level $(0.19 \mathrm{mg} / \mathrm{mL})$, and up to 4 times the amount at the highest cholesterol level $(0.57 \mathrm{mg} / \mathrm{mL})$, than non-induced cells. Similarly, cholesterol reduction in milk was enhanced when milk was inoculated with induced cells of $R$. equi, although the increased cholesterol degradation by induced cells was only evident after $7 \mathrm{~d}$ incubation.

The results of the present work suggest that, like the COD from Pseudomonas fluorescens, the COD from Rhodococcus equi No.23 may have application to the commercial preparation of reduced cholesterol egg-based products for the food industry. The COD from $P$. fluorescens was found to degrade cholesterol at temperatures as low as $4^{\circ} \mathrm{C}$; the ability of the COD from $R$. equi to degrade cholesterol at low temperatures remains to be investigated. 


\section{Bibliography}


ABS (1993) Apparent consumption of selected foodstuffs, Australia 1990-91 Preliminary Cat. No. 4306.0 Canberra: Commonwealth Government printer.

Ahrens, E. H. (1985) The diet-heart question in 1985: Has it really been settled? The Lancet, 1085-1087.

Aihara, H., K. Watanabe, and R. Nakamura (1988a) Degradation of cholesterol in egg yolk by Rhodococcus equi No. 23 J. Food Sci. 53, 659-660.

Aihara, H., K. Watanabe and R. Nakamura (1988b) Degradation of cholesterol in hens egg yolk and its low density lipoproteins by extracellular enzymes of Rhodococcus equi No. 23 Lebensm-Wiss. u. -Technol. 21, 342-345.

Aihara, H., K. Watanabe and R. Nakamura (1986) Characterization of production of cholesterol oxidase in three Rhodococcus strains J. Applied Bacteriology, 61, 269-274.

Al-Hasani, S., H. Shabany and J. Hlavac (1990) Rapid determination of cholesterol in selected frozen foods. J. Assoc. Offic. Anal. Chem. 73, 817820.

Allain C. C., L. S. Poon, C. S. G. Chan, W. Richmond and P. C. Fu (1974) Enzymatic determination of total serum cholesterol Clin. Chem. 20, 470-475. 
Andrews, J. W., R. K. Wagstaff and H. M. Edwards (1968) Cholesterol metabolism in the laying fowl Am. J. Physiol. 214, 1078-1083.

Anon. (1996) Cholesterol concerns now are lower Poultry Digest June, 38.

Anon. (1991) 'Catalyst' in Today's Life Science 3, 6.

Anon. (1988) Report of the National Cholesterol Education Program Expert Panel on detection, evaluation and treatment of high blood cholesterol in adults Arch. Intern. Med. 148, 36-69.

Anon. (1984) The Lipid Research Coronary Clinics Primary Prevention Trial results. II. The relationship of reduction in incidence of coronary heart diseases to cholesterol lowering JAMA 251, 365-374.

Anon. (1973) Foods of tomorrow Food Proc. 27, 57.

Arima, K., M. Bae, M. Nagasawa and G. Tamura (1969) Microbial transformation of sterols Part I. Decomposition of cholesterol by micro-organisms Agric. Biol. Chem. 33, 1636-1643.

Awad, A. C., M. R. Bennink and D. M. Smith (1997) Composition and functional properties of cholesterol reduced egg yolk Poultry Sci. 76, 649-658. 
Awad, K., M. Bae, M. NagAsawa and G. Tamura (1969) Microbial transformation of sterols Part I Decomposition of cholesterol by micr-organisms Agric. Biol. Chem. 33, 1636-1643.

Bair, C. W. and W. W. Marion (1978) Yolk cholesterol in eggs from various avian species Poultry Sci. 57, 1260-1265.

Bartov I. and R. Reiser (1973) Relationship between plasma cholesterol levels and fecal steroid excretion of cockerels fed low-cholesterol containing diets Poultry Sci. 52, 992-997.

Beitz, D. C., J. W. Young and S. S. Dehal (1990) Method for converting cholesterol in food to coprostanol US Patent 4,921,710.

Bender, H. (1986) Production, characteristisation, and application of cyclodextrins. Adv. Biotechnol. Proc. 6, 31.

Berezkin, V. C. (1983) Chemical methods in gas chromatography Elsevier, Amsterdam, p. 47.

Best, D. (1989) Dairy processors milk new technology Prepared Foods 158, $76,78$.

Beyer, S. R. and L. S. Jensen (1992) Metabolism and nutrition: Cholesterol concentration of egg yolk and blood plasma and performance of laying hens as influenced by dietary a-ketoiscoproic acid. Poultry Sci. 71, 120-127. 
Beyer, J. D., F. X. Milani, M. J. Dutelle and R. L. Bradley Jr. (1989) Gas chromatographic determination of cholesterol in egg products. J. Assoc. Offic. Anal. Chem. 72, 746-748.

Beyer, R, S. and L. S. Jensen (1989a) Over estimation of the cholesterol content of eggs J. Agric. Food Chem. 37, 917-920.

Beyer, S. R. and L. S. Jensen (1989b) Research note: Cholesterol content of commercially produced eggs in Georgia Poultry Sci. 68, 1703-1706.

Beynen, A. C. and M. B. Katan (1985) Reproducibility of the variations between humans in the response of serum cholesterol to cessation of egg consumption A therosclerosis Oct. 57, 19-31.

Beynen, A. C. and M. B. Katan (1983) Possible mechanisms underlying hyperand hypo-responsiveness to dietary cholesterol in man Fat Science Proc. 16th Congress, Budapest, 1089-1103.

Bitman, J. and D. L. Wood (1980) Cholesterol and cholesterol ester of eggs from various avian species Poul. Sci. 59, 2014.

Brown, H. G. (1987) Adaptation of an HPLC system to quantify cholesterol JAOCS 64, 106-108. 
Brown, M. S. and J. L. Goldstein (1984) How LDL receptors influence cholesterol and atherosclerosis. Bri. Am. 251151, 58-66.

Burgess, T. L., C. Burgess and J. D. Wilson (1962) Effects of MER-29 on egg production in the chicken Proc. Soc. Exp. Biol. Med. 109, 218-221.

Chambaz, E. M. and E. C. Horning (1969) Conversion of steroids to trimethylsilyl derivatives for gas phase analytical studies: Reactions of silylating reagents Anal. Biochem. 30, 7-24.

Cheetham, P. S. J., P. Dunil and M. D. Lilly (1982) The characterization and interconversion of three forms of cholesterol oxidase extracted from Nocardia rhodochrous Biochemical J. 201, 515-521.

Cheillan, F., H. Lafont, E. Termine, F. Fernandez, P. Sauve and G. Lesgards (1989) Molecular characteristics of the cholesterol oxidase and factors influencing it's activity Biochim. Biophys. Acta 999, 233-238.

Chicoye, E., W. D. Powrie and O. Fennema (1968) Photoxidation of cholesterol in spray-dried egg yolk upon irradiation J. Food Sci. 33(6), 581-587.

Chobert, J. M., C. Bertrand-Harb and M. G. Nicolas (1988) Solubility and emulsifying properties of caseins and whey proteins modified enzymaically by trypsin J. Agric. Food Chem. 26, 883. 
Clarenburg, R., I. A. K. Chung and L. M. Wakefield (1971) Reducing the egg cholesterol level by including emulsified sitosterol in standard chicken diet $\mathbf{J}$. Nutr. 101, 289-298.

Consensus Conference (1985) Lowering blood choleterol to prevent heart disease. J. Am. Med. Ass. 253, 2080-2086.

Cook, F. and G. M. Briggs (1977) Nutritive value of eggs in Egg Science and Technology 2nd ed. Stadelman, W. J., Cotterill, O. J. (eds), AVI: Westernport CT, pp. 92-108.

Corday, E. and S. R.Corday (1975) Editorial: Prevention of heart disease by control of risk factors: the time has come to face the facts Am J. Cardiol. 35(2), 330-333.

Crawford, M. H (1990) A new, improved egg? Science 248 (4952), 166.

Csallany, A. S., S. E. Kindom, P. B. Addis and J. Lee (1989) HPLC method for quantitation of cholesterol and four of it's major oxidation products in muscle and liver tissues Lipids 24, 645-651.

Cully, J and H. R. Vollbrecht (1992) Process for manufacture of cholesterol reduced egg yolk Patent DE 4029287 A1.

Cully, J and H. R. Vollbrecht (1991) Process for removal $\beta$-cyclodextrin from egg yolk Patent DE $4001611 \mathrm{Cl}$. 
Davidson, S. (1990) Low-cholesterol milk and eggs Rural research, 148, 2728.

Dehal, S. S., B. A. Osslund, J. W. Young and D. C. Beitz (1991) Partial purification of cholesterol reductase from alfalfa leaves Medicago-Sativa 75th Annual meeting of the Federation of American Societies for Experimental Biology, Atlanta Georgia USA April 21-25 FASEB (Fed. Am. Soc. Exp. Biol.) 5, Abs No. 304.

Dehal, S. S., J. W. Young and D. C. Beitz (1990) Demonstration of cholesterol reductase in pea (Pisum sativum) leaves 74th Annual conference of the Federation of American Societies for Experimental Biology Part 1 April 1-5 Washington D.C. USA FASEB (Fed. Am. Soc. Exp. Biol.) 4, Abs No. 1552.

Dehal, S. S., D. C. Beitz and J. W. Young (1988) Discovery of cholesterol reductase activity in plant leaves 72nd Annual meeting of the Federation of American Societies for Experimental Biology, Las Vegas Nevada USA May 1-5 FASEB (Fed Am. Soc. Exp. Biol.) 2, A534.

Dziezak, J. D. (1991) Enzymes: Catalysts for food processes Food Technol. 7885.

Edwards, H. M. Jr., J. F. Marion and J. C. Driggers (1962) Serum and egg cholesterol levels in mature hens as influenced by dietary protein and fat changes. Poultry Sci. 41, 713-717. 
Elkin, R. G., M. B. Freed, K. A. Kieft and R. S. Newton (1993) Alteration of egg yolk cholesterol content and plasma lipoprotein profiles following administration of a totally synthetic HMG-CoA reductase inhibitor to laying hens J. Agric. Food Chem. 41, 1094-1101.

Elkin, R. G. and J. C. Roger (1990) Reduction of cholesterol content of eggs by the oral administration of lovastatin to laying hens J. Agric. Food Chem. 38, 1635-1641.

Evans, R. J., D. H. Bauer, S. L. Bandeman, S. B. Vaghefiand C. J. Flegal (1973) Structure of egg yolk very low density lipoprotein. Polydispersity of the very low density lipoprotein and the role of lipovitellin in the structure Arch. Biochem. Biophys. 154, 493.

Fairchild, P. B. (1991) The food and drug administration looks at cholesterol J. Assoc. Food Drug Officials 55, 41-44.

Fakuda, H., Y. Kawakami and S. Nakamura (1973) A method to screen anticholesterol substances produced by microbes and a new cholesterol oxidase produced by Streptomyces violascens. Chemical and Pharmaceutical Bulletin 21, 2057-2060.

Favati, F., J. W. King, J. P. Friedrich and K. Eskins (1988) Supercritical $\mathrm{CO}_{2}$ extraction of carotene and lutein from leaf protein concentrates. J. Food Sci. 53, 1532-1536. 
Feeley, R. M., P. E. Criner and B. K. Watt (1972) Cholesterol content of foods J.Am. Diet. Assoc. 61, 134-148.

Ferriera, N. P. and R. P. Tracey (1984) Numerical taxonomy of cholesteroldegrading soil bacteria J. App. Bact. 57, 429-446.

Flegg, H. M. (1973) An investigation of the determination of serum cholesterol by an enzymatic method Ann. Clin. Biochem. 10, 79.

Flynn, M. A., Nolph, T. C. Flynn., R. Kahrs, and G. Krause (1979) Effect of dietary eggs on human serum cholesterol and triglycerides Am. J. Clin. Nutr. $32,1051-1057$.

Froning, G. W., R.L. Wehling, S. L. Cuppett, M. M. Pierce, L. Niemann and D. K. Siekman (1990) Extraction of cholesterol and other lipids from dried egg yolk using supercritical carbon dioxide J. Food Sci. 55, 95-98.

Gleispach, H. (1974) The use of different silylating agents for structure analysis of steroids J. of Chromat. 91, 407-412.

Goodfellow, M. (1984) Genus Rhodococcus Zopf 1891, 28AL in Peter H. A. Sneath (ed.) Bergeys Manual of Systematic Bacteriology Vol. 2, Williams and Wilkins, Baltimore 1472. 
Gordon, D. J., J. Knoke, J. L. Probstfield, R. Superko and H. A. Tyroler (1986) High density lipoprotein cholesterol and coronary heart disease in hypercholesterolemic men: the lipid research clinics coronary primary prevention trial Circulation 74, 1217-1225.

Gornall, D. A. and A. Kuksis (1971) Molecular species of glycerophosphatides and triglycerides of egg yolk lipoproteins Can. J. Biochem. 49, 51-60.

Gotto, A. M. and M. D. Phil (1984) Directions of atherosclerosis research in the 1980's and 1990's Circulation 70 (suppl III), 89-94.

Gross, A. (1991) Enzymatic catalysis in the production of novel food ingredients Food Technol. 96-97, 100.

Gurr, M. I., N. Borlak and S. Ganatra (1989) Dietary fat and plasma lipids Nutrition Research Reviews 2, 63-86.

Haddadin, M. S. Y., S. M. Abdulrahim, E. A. R. Hashlamoun and R. K. Robinson (1996) Effect of lactobacillus acidophilus on the production and chemical composition of hens eggs Poultry Sci. 75, 491-494.

Hamada, J. S. and W. E. Marshall (1989) Preparation of functional properties of enzymatically deamidated soy proteins. J. Food Sci. 54, 598-601, 635.

Hargis, P. S. (1988) Modifying egg yolk cholesterol in the domestic fow-a review Worlds Poultry Sci. J. 44, 17-29. 
Heftman, E. and I. R. Hunter (1979) High pressure liquid chromatography of steroids J. of Chrom. 165, 283-299.

Hegenbart, S. (1989) Cholesterol: Consumer interest rises Dairy Foods 90, 145146.

Hegsted, D. M. (1986) Serum-cholesterol response to dietary cholesterol: a reevaluation Am J. Clin. Nutr. 44, 299-305.

Helwig, L. R. Jr., E. J. Mulnix and J. M. Regenstein (1978) Effects of varied zinc/copper ratios on egg and plasma cholesterol level in white leghorn hens. J. Food Sci. 43, 666-669.

Holden, J., J. Exler, C. McCharen, J. Lockard (1989) A nationwide study of the cholesterol, proximate, vitamin, and mineral levels in large eggs FASEB (Fed Am. Soc. Exp. Biol.) 3, A658.

Holdsworth, G. and J. B. Finean (1972) A structural study of the low-density lipoprotein fraction from hen's eggs and of an isolated surface component Chem. Phys. Lipids 9, 217-219.

Horvath, C. (1966) Quantitative determination of cholesterol in autoxidation mixtures by thin-layer chromatography. J. Chromatog. 22, 52-59. 
Hung, T. V., and M. Unger (1993) Production of cholesterol reduced egg products by supercritical fluid extraction AIFST 26th Convention Proceedings 2nd-6th May, Adelaide Symposium 11.8.

Hutchinson, G. I., H. Greenfield and R. B. H. Wills (1987) Composition of Australian Foods, 35. Pork Food Technol. Aust. 39, 216-222.

Ikawa, S., M. Takita and M. Ogura (1979) Steroids as substrates for cholesterol oxygen oxidoreductase, with special reference to $3 \beta$-hydroxy bile acids.J. Biochem. 85, 1447-1452.

Inouye Y., K. Taguchi, A. Fujii, K. Ishimaru, S. Nakamura and R. Nomi (1982) Purification and characterisation of extracellular $3 \beta$-hydroxysteroid oxidase produced by Streptoverticillium cholesterolicum. Chem. Pharm. Bull. 30, 951958.

Jellema, A. (1980) The action of milk lipase on milkfat globules: physiological and mechanical factors Neth. Milk Dairy J. 34, 133-135.

Jennings, W. G. ed. (1981) Applications of glass capillary gas chromatography, Chromatographic Science series Volume 15, M. Dekker. New York, pp. 221-222

Johnson, T. L. and G. A. Somkuti (1990) Properties of cholesterol dissimilation by Rhodococcus equi J. Food Protection 53, 332-335, 350. 
Kannel, W. B. and T. Gordon (eds) (1974) The Framingham study, an epidemiological investigation of cardiovascular disease, Sect. 26 DHEW (NHI), Washington DC, GPO.

Kannel, W. B., W. P. Castelli and T. Gordon (1979) Cholesterol in the prediction of atherosclerosis disease. New perspectives based on the Framingham study Ann. Int. Med. 90, 85-91.

Karkalas, J., A. E. Donald, K. M. Clegg (1982) Cholesterol content of poultry meat and cheese determined by enzymtic and gas-liquid chromatography methods J. Food Technol. 17, 281-283.

Kenney, W. C. and Thomas P. Singer (1979) Identification of the covalently bound flavin prosthetic group of cholesterol oxidase J. Biol. Biochem. 254, 4689-4690.

Kern, F. (1991) Normal Plasma cholesterol in 88 year old man who eats 25 eggs per day New England J. Med. 324, 896-899.

Kestin, M., P. M. Clifton, I. L. Rouse and P. J. Nestel (1989a) Effect of dietary cholesterol in normolipidemic subjects is not modified by nature and amount of dietary fat. Am. J. Clin. Nutr. 50, 528-532. 
Kestin, M., I. L. Rouse, R. A. Correll and P. J. Nestel (1989b) Cardiovascular disease risk factors in free-living men: comparison of two prudent diets, one based on lactoovovegetarianism and the other allowing lean meat Am. J. Clin. Nutr. $50,280-287$.

Keys, A. (1984) Serum cholesterol response to dietary cholesterol. Am. J. Clin. Nutr. 40, 351-359.

Kindel S. (1981) Enzymes, the bio-industrial revolution Technology 1, 62.

Kleman, L. P. and J. W. Finley (1991) Process for separation of sterol compounds from fluid mixtures. United States Patent us 5064 668, 16pp. [En] Nabisco Inc., Parsippany, NJ, USA.

Klevay, L. M. (1973) Hypercholesterolaemia in rats produced by an increase in the ratio of zinc to copper ingested Am. J. Clin. Nutr. 26, 1060-1068.

Kondra, P. and G. C. Hodgson (1961) Genetic differences in energy protein requirements of chicks. Poultry Sci. 40, 525-531.

Konlande, J. E. and H. Fisher (1969) Evidence for a non-absorbable antihypercholesterolemic action of phytosterols in the chicken J. Nutr. 98, 435-442.

Korahni, V., J. Bascoul and A. Crastes de Paulet (1981) Capillary column gasliquid chromatographic analysis of cholesterol derivatives. Application to the autoxidation products of cholesterol. J. Chromatog. 211, 392-397. 
Kou, I and R. P. Holmes (1985) The analysis of 25-hydroxycholesterol in plasma and cholesterol-containing foods by high-performance liquid chromatography. J. Chromatog. 330, 339-346.

Kovacs, M. I. P. (1990) Determination of cholesterol in pasta products using gasliquid chromatography J. Cereal Sci. 11, 291-297.

Kronmal, R. (1985) Commentary on the published results of the lipid research clinics Coronary Primary Prevention Trial JAMA 253, 2091-2093.

Kudochodkar, B. J., L. Horlick and J. B. O' Neil (1976) Absorption of dietary beta-sitosterol in laying hens and it's incorporation into the egg J. Nutr. Nov. 106(11), 1629-1636.

Kuksis, A. (1992) Yolk Lipids Biochim. Biophys. Acta Mar. 25, 1124(3), 205-222.

Kuksis, A., O. Stachnyk and B. J. Holub (1969) Improved quantitation of plasma lipids by direct gas-liquid chromatography. J. Lipid Res. 10, 661-667.

Kumar, N. and O. P. Singhal (1991) Cholesterol Oxides and Atherosclerosis: A Review J. Sci. Food Agric. 55, 497-510.

Kushiro, H., J Nakamoto, I. Fukui, Z. Ogawa, Y. Yamaguchi, K. Arisue, C. Hayashi and Y. Yamamura (1982) Cholesterol content of foodstuffs Asian Medical Journal 25, 69-74. 
Larsen, J. E. and G. W. Froning (1981) Extraction and processing of various components from egg yolk Poultry Sci. 60, 160-167.

Leblond, D. (1990) Technology helps firms meet biotech challenge European Chem. News June 4, 24.

Lebovics, V. K., O. Gaal, L. Somogyi and J. Farkas (1992) Cholesterol oxides in g-irradiated spray-dried egg powder J. Sci. Food Agric. 60, 251-254.

Lehninger, A. L. (1975a) Biochemistry 2nd ed., Worth publishers Inc., New York, p. 189.

Lehninger, A. L. (1975b) Biochemistry 2nd ed., Worth publishers Inc., New York, p. 685.

Levy, R. I. (1985) Primary prevention of coronary heart disease by lowering lipids: results and implications Am. Heart J. 110, 1116-1122.

Li, L., T. A. Freier, P. A. Hartman, J. W. Young and D. C. Beitz (1992) Conversion of cholesterol to coprostanol by Eubacterium sp. strain HL 72nd Annual meeting of the Federation of American Societies for Experimental Biology, Part 1 Anaheim California USA April 5-9 1992 FASEB (Fed. Am. Soc. Exp. Biol.) 6, A1389. 
Lirette A., A. R. Robinson, D. C. Crober, P. D. Lawson and N. L. Firth (1993) Effect of oat bran, cotton seed hulls and guar gum on chicken egg and blood lipids during the early laying period Canadian J. Anim. Sci. 73, 673-677.

Loomis, D. W., J. D. Lile, R. P. Sandstrom and A. J. Burbott (1979) Adsorbent polystyrene as an aid in plant enzyme isolation Phytochemistry 18, 1049-1054.

Luukkainen, T., W. J. A. Vandenheuvel, E. O. A. Haahti and E. C. Horning (1961) Biochim. Biophys. Acta 52, 559.

Maerker, G., and J. Unruh Jr. (1986) Cholesterol oxides I. Isolation and determination of some cholesterol oxidation products JOACS 63, 767-771.

Marks, H. L. and K. W. Washburn (1977) Divergent selection for yolk lipid and reproductive efficiency of the hen. Br. Poult. Sci. 18, 179-188.

Martin, W. G., N. H. Tattrie and W. H. Cook (1963) Lipid extraction and distribution studies of egg tolk lipoproteins. Can. J. Biocem. Physiol. 41, 657-666.

Masoom and Townsend (1985) Determination of cholesterol by flow injection analysis with immobilised cholesterol oxidase. Analytica Chimica Acta 174, 293-297. 
Mattson F. H. and S. M. Grundy (1985) Comparison of effects of dietary saturated, monounsaturated, and polyunstaurated fatty acids on plasma and lipoproteins in man. J. Lipid Res. 26, 194-202.

Maurice, D. V., S. F. Lightsey, K. t. Hsu, T. G. Gaylord and R. V. Reddy (1994) Cholesterol in eggs from dfferent species of poultry determined by capillary GLC Food Chem. 50, 367-372.

McDonald, M. W. and T. Shafey (1989) Nutrition of the hen and egg cholesterol Proceedings, Cholesterol in eggs seminar Melb., 30-34.

McGilvery, R. W. (1979) Biochemistry, a functional approach 2nd ed., W. B. Saunders Co., Philadelphia, pp. 253-256, 666, 674.

McNaughton, J. L. (1978) Effect of dietary fibre on egg yolk, liver, and plasma cholesterol concentrations of the laying hen J. Nutr. 108, 1842-1848.

Mohan, B., R. Kadirvel, M. Bhaskaran and A. Natarajan (1995) Effect of probiotic supplementation on serumbyolk cholesterol and on egg shell thickness in layers Br. Poultry Sci. 36, 799-809.

Moore, N. F., J. Patzer, Y. Barenholz and R. R. Wagner (1977) Effect of phospholipase $C$ and cholesterol oxidase on membrane integrity, microviscosity, and infectivity of vesicular stomatitis virus Biochemistry 16 (21), 4709-4715. 
Morgan, J. N. and D. J. Armstrong (1989) Wide-bore capillary gas chromatographic method for quantification of oxidised cholesterol derivatives in eg yolk powder J. Food Sci. 54, 427.

Naber, E. C. (1991) Cholesterol content of eggs: Can and should it be changed? Adv. Appl. Biotechnol. Ser. 12 (Fat and cholesterol reduced foods), 261276.

Naber, E. C. (1983) Nutrient and drug effects on cholesterol metabolism in the laying hen Fed. Proc. 42, 2486-2493.

Naber, E. C. and M. D. Biggert (1985) Analysis for generation of cholesterol oxidation products in egg yolk by heat treatment Poult. Sci. 64, 341-347.

Naber, E. C., J. F. Elliot ans T. L. Smith (1982) Effect of probucol on reproductive performance, egg yolk cholesterol content and lipid metabolism in the laying hen. Poult. Sci. 61, 1118-24.

National Heart Foundation of Australia (NHFA) (1989) Update on diet and heart disease

Neidlemann, S. L. (1991) Enzymes in the food industry: a backward glance Food Technol. 88-91. 
Nestel, P. J., M. Kestin, P. M. Clifton (1989) Effects of dietary cholesterol in human subjects: Interactions between egg yolk cholesterol and the type of dietary fat Proceedings, Cholesterol in eggs seminar Melb., 19-27.

NHMRC (1992) Report of the NHMRC Working Party: Polyunsaturated fats in the Australian diet Food Aust. 44, 235-236.

Noma, A. and K. Nakayama (1976) Comparative studies on cholesterol oxidases from different sources. Clinica Chimica Acta 73, 487-496.

O'Dea, K. and A. J. Sinclair (1991) Eggs: implications for human health in Australian Poultry Science Symposium, Sydney 23-28.

O'Dea, K. and A. J. Sinclair (1989) Effect of one or two eggs daily on plasma cholesterol and triglyceride levels: Interaction with other dietary fats Proceedings, Cholesterol in eggs seminar Melb., 19-27.

O'Dea, K. K. Traianedes, N. K. Chisolm, H. Leydem and A. J. Sinclair (1991) Cholesterol-loweing effect of a low-fat diet containing lean beef is reversed by the addition of beef fat Am. J. Clin. Nutr. 52, 491-494.

Osterberg, E., A-C Blomstrom and K, Hulmberg (1989) Lipase catalysed transesterification of unsaturated lipids in a microemulsion J. Am. Oil Chem. Soc. 66,1330 . 
Owen, R. W., A. N. Mason and R. F. Billton (1983) The degradation of cholesterol by Pseudomonas sp. NCIB 10590 under aerobic conditions J. Lipid Research 24, 1500-1511.

Penet, C. S. (1991) New applications of industrial food Enzymology: Economics and processes Food Technol. 98-99.

Pratt, D. E. (1975) Lipid Analysis of a frozen egg substitute. J. Am. Diet Assoc. 66, 31-33.

Prescott, J. F., M. Lastra and L. Barksdale (1982) Equi factors in the identification of Corynebacterium equi Manusson J. of Clin. Microbiol. 988-990.

Pszczola, D. E. (1988) Production and potential food applications of cyclodextrins. Food Technol. 42, 96.

Ramesh, B, S. S. Adkar, A. V. Prabhudesai and C. V. Viswanathan (1979) Selective extraction of phospholipids from egg yolk JAOCS 56, 585-587.

Randolph, T. W., D. S. Clark, H. Blanch and Prausnitz (1988) Enzymatic oxidation of cholesterol aggregates in supercritical carbon dioxide. Science 238, 387-390.

Reddy, R. V., S. F. Lightsey and D. V. Maurice (1991) Effect of feeding garlic oil on performance and egg yolk cholesterol concentration Poultry Sci. 70, 20062009. 
Richmond, W. (1973) Preparation and properties of a cholesterol oxidase from Nocardia sp. and it's application to the enzymatic assay of total cholesterol in serum Clin. Chem. 19, 1350-1356.

Rifkind, B. M. (1986) Diet, plasma cholesterol and coronary heart disease J. Nutr. 116, 1578-1580.

Ritchie, A. S. and M. H. Jee (1985) High-performance liquid chromatographic technique for the separation of lipid classes. J. Chromatog. 329, 273-280.

Romanoff, A. L. and A. J. Romanoff (1949) The avian egg. John Wiley and Sons Inc., New York, NY.

Schucker, B., K. Bailey, J. T. Heimbach, M. E. Mattson, J. T Wittes, C. M. Haines, D. J. Gordon, J. A. Cutler, V. S. Keating, R. S. Goor, B. M. Rifkind (1987a) Change in public perspective on cholesterol and heart disease. Results from two national surveys. JAMA 258, 3527-3531.

Schucker, B., J. T. Wittes, J. A. Cutler, K. Bailey, D. R. Mackintosh, D. J. Gordon, C. M. Haines, M. E. Mattson, R. S. Goor and B. M. Rifkind (1987b) Change in physician perspective on cholesterol and heart disease. Results from two national surveys JAMA 258, 3521-3526.

Sheather, K. R. (1992) Successes and failures in Australia Poultry International $31,60-68$. 
Sidhu, G. S. (1989) Cholesterol in dairy foods and the technology for ts removal Proceedings, Developments in milkfat technology seminar Melb. 30, 43.

Sim, J. S., W. D. Kitts and D. B. Bragg (1980) Influence of dietary oil, cholesterol, and soysterols on the feacal neutral and acidic steroid excretion in laying hens Poult. Sci. 59, 325-327.

Sim, J. S. and D. B. Bragg (1978) Effect of dietary oil, cholesterol, and soysterols on the lipid concentration and fatty acid composition of egg yolk, liver and serum of laying hens Poultry Sci. 57, 466-472.

Simons, L. A., Y. Friedlander, J. Simons and J. D. Kark (1988) Familial aggregation of coronary heart disease: partial mediation by high density lipoproteins? Atherosclerosis 69 (2-3), 139-144.

Smith, A. G. and C. J. W. Brooks (1977) The mechanism of the isomerisation of cholest-5-en-3-one to cholest-4-en-3-one by cholesterol oxidase.Biochem. Soc. Trans. 5, 1088-1090.

Smith A. G. and C. J. Brooks (1974) Application of cholesterol oxidase in the analysis of steroids J. Chromatography 101, 373-378.

Snedecor, G. W. and W. G. Cochran (1967) Statistical Methods The Iowa State University Press Ames, Iowa, U.S.A. 6th edn. 
Soehnlen, J. (1987) The diet-heart disease contriversy: new research looks promising for the dairy industry Cultured dairy products journal, 29-33.

Stehbens, W. E. (1990) The Cholesterol-Heart Disease Controversy ASEAN Food J. 5, 131-141.

Sugano, H. and I. Watanabe (1961) Isolations and some properties of native lipoproteins from egg yolk. J. Biochem. 50, 473.

Sutton, C, D, W, M, Muir and G. E. Mitchell, Jr. (1984) Cholesterol metabolism in the laying hen as influenced by dietary cholesterol, caloric intake and genotype Poultry Sci. 63, 972-980.

Swift, M. L. (1984) Analysis of molluscan sterols: calorimetric methods Lipids $19,625-630$.

Tomioka, H., M. Kagawa and S. Nakamura (1976) Some enzymatic properties of 3ß-hydroxysteroid oxidase produced by Streptomyces violascens J. Biochem. 79, 903-915.

Tsai, L. S., K. Ijichi, C. A. Hudson and J. J. Meehan (1980) A method for the quantitative estimation of cholesterol -oxide in eggs Lipids 15, 124.

Tu, C., W. D. Powrie and O. Fennema (1970) Steroids in egg yolk J. Food Sci. $35,601-606$. 
Turfitt, G. E. (1944) Microbiological agencies in the degradation of steroids. I. The cholesterol-decomposing organisms of soils.J. Bacteriol. 47, 487-493.

Turk, D. E. and B. D. Barnett (1972) Diet and egg cholesterol content Poul. Sci. $50,1303-1306$.

Turk, D. E. and B. D. Barnett (1971) Cholesterol content of market eggs Poul. Sci. $50,1303-1306$.

Ulberth and Reich (1992) Gas chromatographic determination of cholesterol in processed foods Food Chem. 43, 387-391.

Uwajima, T., H. Yagi and O. Terada (1974) Properties of crystalline 3ß-hydroxysteroid oxidase of Brevibacterium sterolicum Agricul. Biol. Chem. 38, 11491156.

Vargas, R. E., J. B. Allred, M. D. Biggert and E. C. Naber (1986) Effect of Dietary 7-ketocholesterol, pure, or oxidised cholesterol on hepatic 3-hydroxy-3methylglutaryl-coenzyme A reductase activity, energy balance, egg cholesterol concentration, and ${ }^{14} \mathrm{C}$-acetate incorportaion into yolk lipids of laying hens Poultry Sci. $65,1333-1342$.

Warren, M. W. and H. R. Ball (1990) Yolk or white: Which is more important to egg flavour Egg Industry Sept/Oct., 24-26. 
Warren, M. W., H. G. Brown and D. R. Davis (1988) Solvent extraction of lipid components from egg yolk solids. JAOCS 65, 1136-1139.

Washburn, K. W. and D. F. Nix (1974a) Genetic basis of yolk cholesterol content Poultry Sci. 53, 109-115.

Washburn, K. W. and D. F. Nix (1974b) A rapid technique for extraction of yolk cholesterol Poultry Sci. 53, 1118-1122.

Washburn, K. W., R. A. Guill and H. M. Edwards Jr. (1975) Influence of genetic differences in feed efficiency of young chickens on derivation of metabolizable energy from the diet and nitrogen retention J. Nutr. 105, 726-732.

Watanabe, K., H. Aihara, Y. Nakagawa, R. Nakamura, and T. Sasaki (1989) Properties of the purified extracellular cholesterol oxidase from Rhodococcus equi No. 23 J. Agric. Food Chem. 37, 1178-1182.

Watanabe, K., H. Shimizu, H. Aihara, R. Nakamura, K. Suzuki, and K. Komagata (1986) Isolation and identification of cholesterol-degrading Rhodococcus strains from food of animal origin and their cholesterol oxidase activities J. Gen. Appl. Microbiol. 32, 137-147.

Watkins, B. A. and R. G. Elkin (1992) Dietary modulation of oleic and stearic acids in egg yolk J. Food Comp. Anal. 5, 209-215. 
Weiss, F. G. and M. L. Scott (1979) Effects of dietary fibre, fat and total energy upon plasma cholesterol and other parameters in chickens J. Nutr. 109, 693-701.

Weiss, J. F., E. C. Naber and R. M. Johnson (1967a) Effect of some dietary factors and drugs on cholesterol concentrations in the egg and plasma of the laying hen J. Nutr. 91, 119-128.

Weiss, J. F., E. C. Naber and R. M. Johnson (1967b) Effect of dietary fat and cholesterol in the in vivo incorporation of acetage $-{ }^{14} \mathrm{C}$ into hen liver and ovarian lipids J. Nutr. 93, 142-152.

Weiss, J. F., E. C. Naber and R. M. Johnson (1964) Effect of dietary fat and other factors on egg yolk cholesterol 1. The "cholesterol" content of egg yolk as influenced by dietary unsaturated fat and the method of determination Arch. of Biochem. and Biophys. 105, 521-526.

Williams, A. (1969) Introduction to the chemistry of enzyme action McGrawHill publishing company Ltd, 8

Xiansheng, W., T. V. Hung, P. G. Drew and K. Versteeg (1990) Enzymatic degradation of cholesterol in milk Aust. J. Dairy Technol. 45, 50-52.

Yeh, S. J. and G. A. Leveille (1972) Influence of dietary protein level on plasma cholesterol turnover and faecal steroid excretion in the chick. J. Nutr. 103, 40711. 
Zlatkis, A., B. Zak and A. J. Boyle (1953) A new method for the direct determination of serum cholesterol J. Lab. Clin. Med. 41, 486-492. 


\title{
Enzymatic Degradation of Egg Yolk Cholesterol
}

\author{
SPEROULLA CHRISTODOULOU ${ }^{* 1}$, TRAN V. HUNG ${ }^{1}$, MAURIE A. TREWHELL ${ }^{2}$ and ROBERT G. BLACK ${ }^{2}$
}

\author{
'Food Research Institute, Department of Agriculture, Sneydes Road, Werribee, Victoria, 3030; and \\ 'Victoria University of Technology, Ballarat Road, Footscray, Victoria, 3011
}

(Received November 22, 1993/Accepted May 13, 1994)

\section{ABSTRACT}

The capacity of four cholesterol oxidases from Nocardia erythropolis (Ne), Streptomyces species (Ss), Brevibacterium species (Bs) and Pseudomonas fluorescens (Pf) to degrade cholesterol in egg yolk at 4,25 and $37^{\circ} \mathrm{C}$ has been determined. The effects of enzyme/substrate concentration and incubation temperature on the enzymatic activity of these cholesterol oxidases have also been investigated. The cholesterol degrading capacity of these enzymes follows the sequence $\mathrm{Pf}>\mathrm{Ne}>\mathrm{Ss}>\mathrm{Bs}$. Up to $93.4 \%$ cholesterol was degraded by cholesterol oxidases from $P$. fluorescens and Streptomyces species after $72 \mathrm{~h}$ of incubation at $37^{\circ} \mathrm{C}$. At $4^{\circ} \mathrm{C}, P$. fluorescens cholesterol oxidase could degrade up to $64.9 \%$ cholesterol after $48 \mathrm{~h}$.

Key Words: Enzymatic degradation, eggs, cholesterol.

High cholesterol content in egg is a factor contributing to declining egg consumption (27). A suspected causable association between dietary cholesterol intake and the development of coronary heart disease is still a topic for debate (30). Several studies have also indicated no or insignificant association of cholesterol intake from egg with serum cholesterol $(11,17,32)$. Nevertheless, to address this cholesterol concern, the egg industry has attempted to produce low cholesterol eggs as well as egg products by various approaches.

Genetic selection to develop a chicken line which could produce low cholesterol eggs has only yielded moderate results $(2.4 \%$ cholesterol reduction) $(13,22)$. Modification of the hen's feed by dietary lipid treatment or by adding garlic oil did not influence cholesterol concentration in the egg $(26,36)$. Consequently, cholesterol reduced egg products are prepared by rather complex processes (for example, by partially replacing egg yolk with vegetable oil or water then combining with egg white to meet the market demand) $(4,8)$. The recombined egg does not retain the desirable egg flavor. Therefore, there is a need for a simple technique to produce cholesterol-free or cholesterol reduced egg yolk without losing egg flavor, and as a food ingredient for various egg based products.

Extraction of egg cholesterol using organic solvents has met with limited success due to a large amount of cholesterol remaining in the extracted egg as well as some undesirable effects on the functional properties of the egg yolk (20). Recently, $\beta$-cyclodextrin has been used to remove egg cholesterol by the formation of an insoluble complex with cholesterol or its ester in an aqueous solution. However, even with an additional enzymatic treatment it is still difficult to completely remove all residues of $\beta$ cyclodextrin from the yolk (9). This is a major impediment since $\beta$-cyclodextrin is not an approved food ingredient in some countries.

Supercritical fluid extraction could provide another means to remove egg cholesterol. In spite of the high capital investment required, this process already has several commercial applications in the food industry. Although up to $80 \%$ of egg cholesterol can be removed by supercritical fluid extraction using carbon-dioxide as the fluid, an economical technique to recover the cholesterol from the significant amount of co-extracted egg lipid is required as a prerequisite for commercial feasibility $(12,14)$.

Microbiological degradation of cholesterol by some strains of Nocardia, Rhodococcus, Brevibacterium, Corynebacterium or Streptomyces has been reported $(1,2)$. However, some of these organisms may accumulate steroid intermediates with consequent adverse health effects (3). Some strains which could degrade cholesterol without accumulating steroid intermediates have also been isolated (3). When egg yolk was inoculated with Rhodococcus equi, up to $70 \%$ of cholesterol was degraded with almost no accumulation of steroid intermediates (2).

Johnson and Somkuti also found Rhodococcus strains could reduce up to $80 \%$ of egg cholesterol (16). Although these findings indicate that enzymatic treatment could be used for egg cholesterol degradation, for any commercial application it would be necessary to shorten the degradation time of 3 days reported in these studies.

In a previous study, we reported that cholesterol oxidase could degrade all cholesterol in a buffer system and up to $65 \%$ in milk at low temperature $\left(3\right.$ to $7^{\circ} \mathrm{C}$ ), in less than $24 \mathrm{~h}(36)$.

This paper reports cholesterol degradation activities of cholesterol oxidases originating from four different microorganism genera, Brevibacterium, Nocardia, Pseudomonas and Streptomyces, in an egg yolk system under different environments in order to define the optimal conditions for each enzyme. 


\section{MATERLALS AND METHODS}

Egg yolk.

Yolks and whites from 61 eggs (weighing $55 \mathrm{~g}$ each) were carefully separated. The yolks were combined and mixed until homogenous. A $0.2 \mathrm{~g} / \mathrm{ml}$ yolk suspension in $0.1 \mathrm{M}$ potassium phosphate buffer_(KPB) $\mathrm{pH} 7$ containing $0.02 \%$ thimerosal, as an anti-bacterial agent, was portioned into $20 \mathrm{ml}$ lots and stored at $-4^{\circ} \mathrm{C}$ until required.

\section{Cholesterol oxidases.}

Cholesterol oxidases (COD) derived from Ss, Bs, Ne and Pf were purchased from Sigma Chemical Company, St. Louis, MO as lyophilized powders. Cholesterol oxidases from $\mathrm{Ne}$ was reconstituted in $0.1 \mathrm{M} \mathrm{KPB}, \mathrm{pH} 5$ and the others in the same buffer but at $\mathrm{pH}$ 7. These $\mathrm{pH}$ 's have been reported as being the most stable environment to store these enzymes to prevent appreciable loss of activity (28).

Since the incubation $\mathrm{pH}$ may have a strong effect on the enzymatic activity, the degradation capacity of each enzyme was measured at $\mathrm{pH} 7, \mathrm{a} \mathrm{pH}$ at or close to their optimum physiological $\mathrm{pH}$ based on available literature. Cheillan et al. (6) observed activity by $\mathrm{Pf} \mathrm{COD}$ remaining constant as long as the $\mathrm{pH}$ was maintained between 6.5 and 8.5. Uwajima et al. (33) have shown that the enzyme isolated from Brevibacterium had a broad $\mathrm{pH}$ optimum in the range of $\mathrm{pH} 6.0$ to 8.5 and Noma and Nakayama (23) observed $\mathrm{pH}$ optimum of 7.0 for $\mathrm{Ne}$ and $\mathrm{Bs} \mathrm{COD}$, and $\mathrm{pH} 7.5$ for enzyme from Streptomyces.

For enzyme activity, one enzyme unit ( $1 \mathrm{U})$ is defined as the amount of COD that will promote the conversion of $I \mu \mathrm{M}$ of cholesterol to cholest-4-en-3-1 per min under the prescribed test conditions (๑).

\section{Cholesterol determination.}

Cholesterol was determined by gas chromatography, following a method of Kovacs (18) with some modifications (7), with the principal change being that samples containing I to $2 \mathrm{mg}$ cholesterol were converted to trimethylsilyl (TMS) derivatives prior to gas chromatography, by heating with $100 \mu \mathrm{l}$ MSTFA (Sigma) at $100^{\circ} \mathrm{C}$ for $15 \mathrm{~min}$. Octacosan-1-ol or dihydrocholesterol were used as internal standards. Coefficient of variation for the reproducibility and the mean recovery of the method used ranged from 2.2 to $2.9 \%$ and 90.2 to $95 \%$, respectively.

A 25QC2/BP 10-0.25 capillary column was used in a Hewlett Packard 5890 gas chromatograph. Column temperature was set at $285^{\circ} \mathrm{C}$ and injection temperature at $300^{\circ} \mathrm{C}$. A flame ionisation detector was used, and this was set at $320^{\circ} \mathrm{C}$. A split injection (1 $\mu l)$ was made with a split ratio of 30:1. Flow rate of the carrier gas (He) was $0.8 \mathrm{~mL} / \mathrm{min}$ and the head pressure set at 24 psi. The run time was $17 \mathrm{~min}$, with the retention times of the TMS derivatives of cholesterol and dihydrocholesterol being typically 10 and 10.3 min, respectively.

Sterols were identified by co-chromatography using TMS derivatives of commercially purchased standards (Sigma).

\section{Cholesterol degradation products.}

In order to detect COD products, thin-layer chromatography (TLC) was performed with pre-coated silica Gel $60 \mathrm{~F}_{254}$ plates (Merck). Incubation products were separated with a mixed solvent system (chloroform: diethyl ether [60:40]) at room temperature and spots were made visible by spraying with $2^{\prime}, 7^{\prime}$. dichlorofluorescein and viewed under ultra-violet (UV) light (254 $\mathrm{nm})$. Standards of cholesterol and possible oxidation by-products (5-cholesten-3-one, 4-cholesten-3-one, 25-hydroxycholesterol and 7-ketocholesterol) were purchased from Sigma.

The identification of by-products was confirmed using gas chromatographic retention times in conjunction with mass spectra obtained. Incubation products were extracted with diethyl ether (2 $\mathrm{ml}+\mathrm{l} \mathrm{ml}$ ), the extracts evaporated to dryness and the residues dissolved in chloroform $(500 \mu \mid)$. Samples $(I \mu \mid)$ were then taken for GC-MS using a Hewlett Packard 5890 gas chromatograph fitted with a Hewlett Packard 5971A Mass Selective Detector (MSD). The column and chromatographic conditions were the same as for cholesterol determination.

\section{Enzymatic treatment of egg yolk cholesterol.}

Effect of time and temperature. The effect of incubation time on the cholesterol degrading capacity of each enzyme was initially investigated. Yolk suspensions containing $0.1 \mathrm{~g}$ yolk (approximately $3.9 \mu \mathrm{M}$ cholesterol) were added to tightly capped (teflonlined) $10 \mathrm{ml}$ test tubes and incubated, in triplicate, with $3.9 \mathrm{U}$ of each enzyme at 4,25 and $37^{\circ} \mathrm{C}$. Residual cholesterol was determined at $3,6,9,12,24,48$ and $72 \mathrm{~h}$ by gas chromatography. The experiment was not replicated. Results of this investigation were used to select $12 \mathrm{~h}$ as a suitable time for a more detailed study of incubation temperature.

To four of seven test-tubes containing $0.1 \mathrm{~g}$ yolk in buffer suspension, 3.9 $\mathrm{U}$ of COD enzyme from either one of four types (Ne, Ss, Bs and Pf) was added. The other three test-tubes contained no enzyme and were treated as controls. These treatments were randomly positioned into test-tube racks, and these racks were randomly assigned to incubators at each of the temperatures, $5,15,25,37,45$ and $60^{\circ} \mathrm{C}$. Incubation time was $12 \mathrm{~h}$.

The experiment was replicated on three different days and described as a split-plot type design (29). Days were considered as blocks, incubators as plots and test-tubes containing yolks as the sub-plots. Temperature was the main plot treatment and the enzymes (including controls) were the sub-plot treatments. The main temperature effect, and the temperature by enzyme type interaction, were divided into orthogonal polynomial components (29) up to cubic order to determine if there were linear and quadratic trends. The data was analyzed as a temperature by enzyme type factorial with the control being considered in the analysis as an enzyme type. Pairs of means were compared using Fisher's least significant difference (LSD) at the 5\% level.

Effect of concentration. Yolk suspensions containing $0.1 \mathrm{~g}$ yolk cholesterol were incubated with $0,0.5,1,2,4$ and $8 \mathrm{U}$ of enzyme per $\mu \mathrm{M}$ egg cholesterol for $2 \mathrm{~h}$ at $45^{\circ} \mathrm{C}$ and residual cholesterol was determined. The analysis was replicated on 3 days, with each day considered as a block (29). Within each block there was one test-tube sample of each enzyme type by concentration combination, plus two control samples (without enzyme). The arrangement of the test-tubes placed in the incubator was fully randomized. The data was analyzed as an enzyme type by concentration factorial plus an external non-enzyme control treatment, with blocking for time. Within the factorial, the concentration effects and concentration by enzyme type interaction were divided into orthogonal polynomial components (29) up to cubic order to determine if there were linear and quadratic trends. Pairs of means were compared using LSD at the 5\% level.

\section{RESULTS AND DISCUSSION}

Cholesterol content of treated and untreated egg yolks were compared and results expressed as either percent of cholesterol reduction or $\mathrm{mg} / \mathrm{g}$ residual cholesterol.

\section{Effect of time and temperature.}

The time course of cholesterol degradation of four COD in egg yolk at 37, 25 and $4^{\circ} \mathrm{C}$ are shown in Fig. 1, 2 and 3 when the yolk was incubated with the enzymes for $72 \mathrm{~h}$.

The cholesterol degradation activity of each enzyme at $37^{\circ} \mathrm{C}$ is presented in Fig. 1 . At $37^{\circ} \mathrm{C}$, the Bs COD could 


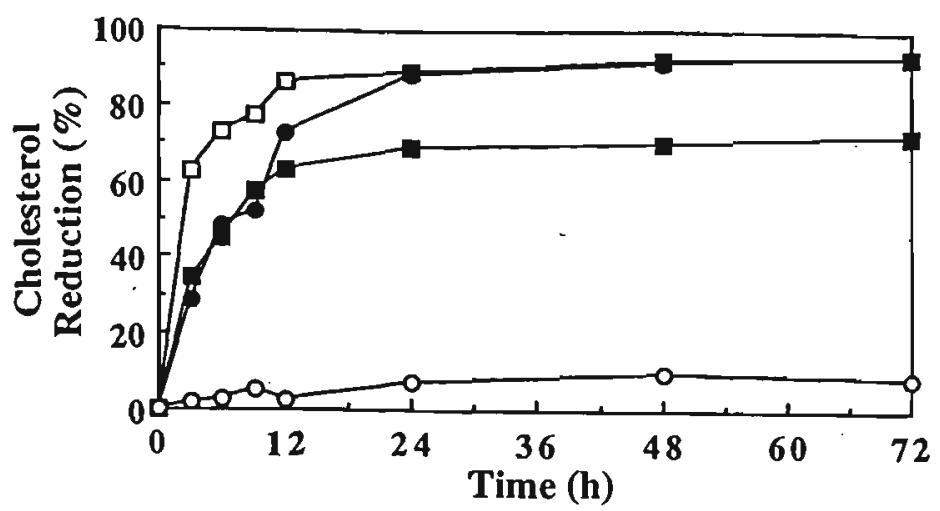

Figure 1. Time course of cholesterol degradation in egg yolk at $37^{\circ} \mathrm{C}$ by $\mathrm{COD}$ of $\mathrm{Pf}(\square), \mathrm{Ne}(\square), \mathrm{Ss}(\mathrm{O})$ and $\mathrm{Bs}(\mathrm{O})$.

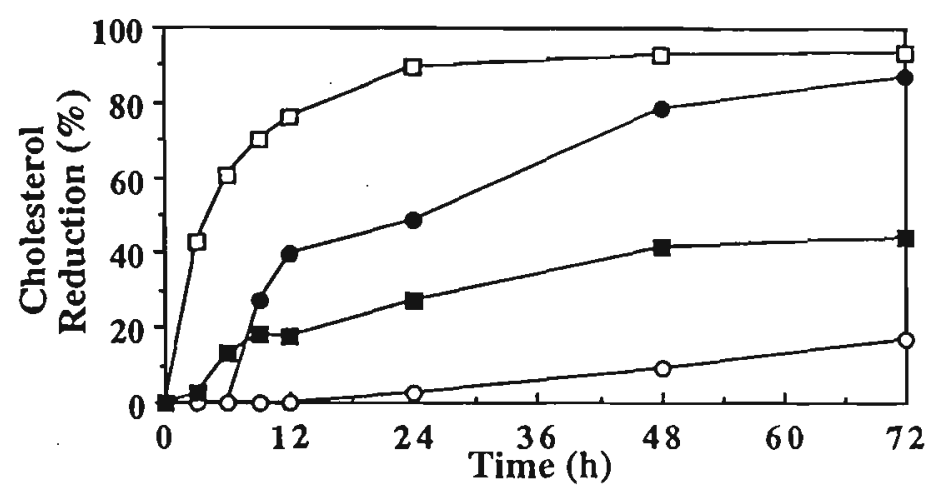

Figure 2. Time course of cholesterol degradation in egg yolk at $25^{\circ} \mathrm{C}$ by $\mathrm{COD}$ of $\mathrm{Pf}(\square), \mathrm{Ne}(\mathrm{\square}), \mathrm{Ss}(\mathrm{O})$ and $\mathrm{Bs}(\mathrm{O})$.

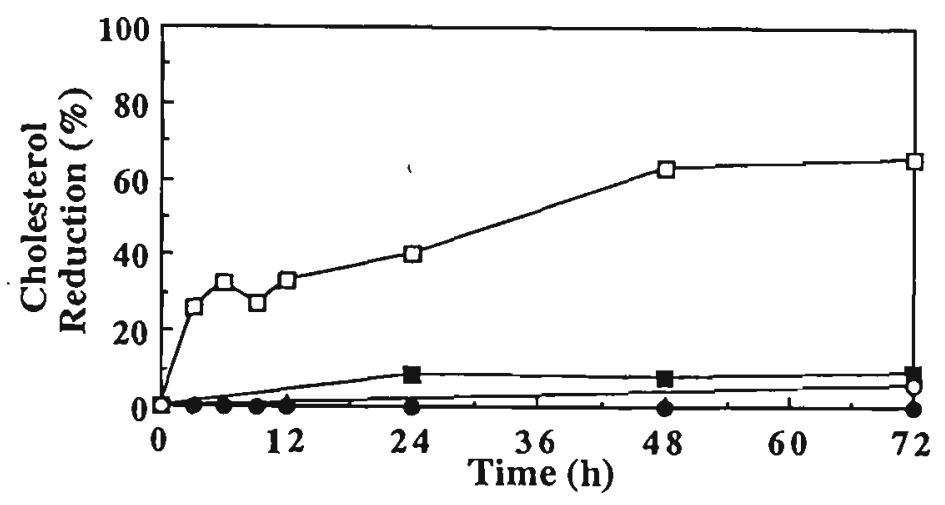

Figure 3. Time course of cholesterol degradation in egg yolk at $4^{\circ} \mathrm{C}$ by $\mathrm{COD}$ of $\mathrm{Pf}(\square), \mathrm{Ne}(\square), \mathrm{Ss}(O)$ and $\mathrm{Bs}(\mathrm{O})$.

only degrade about $8.3 \%$ cholesterol after $72 \mathrm{~h}$ of incubation while Ss COD reduced cholesterol $72.6 \%$. Both Pf $\mathrm{COD}$ and Ne COD, respectively, reduced up to $93.4 \%$ of the cholesterol.

With the exception of Bs COD, cholesterol degradation at $37^{\circ} \mathrm{C}$ occurred in two stages. In the first stage, the reaction proceeded rapidly up to approximately $12 \mathrm{~h}$. In the second stage, the reaction rate became slower, and after $24 \mathrm{~h}$ remained almost unchanged.

The performance of the Pf COD at $25^{\circ} \mathrm{C}$ (Fig. 2) was similar to that at $37^{\circ} \mathrm{C}$ in terms of reaction rate and cholesterol degradation capacity. The reaction of both $\mathrm{Ne}$ and Ss CODs occurred at a much lower rate for the first 48 h at $25^{\circ} \mathrm{C}$ as compared to that at $37^{\circ} \mathrm{C}$. The cholesterol degradation capacity of both $\mathrm{Ne}$ and $\mathrm{Ss}$ CODs also decreased at $25^{\circ} \mathrm{C}$ with $\mathrm{Ne}$ COD degrading $86.4 \%$ and Ss
COD $42.3 \%$ cholesterol after 72 h. Brevibacterium species cholesterol oxidase showed no reaction after $48 \mathrm{~h}$ incubation and $15.7 \%$ after $72 \mathrm{~h}$ incubation. At $4^{\circ} \mathrm{C}$, all enzymes, except Pf COD, showed no or insignificant reaction even after $72 \mathrm{~h}$ of incubation (Fig. 3). Interestingly, Pf COD could degrade approximately $30 \%$ cholesterol within $3 \mathrm{~h}$ and $67.2 \%$ after $48 \mathrm{~h}$, after which the cholesterol level remained almost unchanged.

In a previous study, Ss COD completely degraded cholesterol in a buffer system within $24 \mathrm{~h}$ at 25 and $37^{\circ} \mathrm{C}$ but only $60 \%$ of cholesterol at 7 or $45^{\circ} \mathrm{C}$ (36). In milk, the Ss enzyme reduced about $35 \%$ of cholesterol after $10 \mathrm{~h}$ at $25^{\circ} \mathrm{C}$ but up to $50 \%$ at 3 and $7{ }^{\circ} \mathrm{C}$. By contrast, in the present egg yolk system Ss COD showed high activity at $37^{\circ} \mathrm{C}(65 \%)$ and $25^{\circ} \mathrm{C}(35 \%)$ but no activity at $4^{\circ} \mathrm{C}$. This observation may reflect the effect of substrate environment on the activity of an enzyme.

In a buffer system, Ss oxidases will readily reduce free cholesterol (100\% reduction), but the membrane bound cholesterol present in milk is more difficult to reduce (35 to $50 \%$ reduction) (15). At the most active range of temperature $\left(25\right.$ to $\left.37^{\circ} \mathrm{C}\right)$, Ss COD reacted with egg cholesterol as in a buffer system. At $4^{\circ} \mathrm{C}$, contrary to its behavior with milk cholesterol, the Ss enzyme failed to react with egg cholesterol, possibly due to a lack of a common bonding site for both enzyme and cholesterol, which appears to be present in the milkfat membrane at low temperatures (36). The results show that the capacity for yolk COD by these enzymes followed the sequence $\mathrm{Pf}>\mathrm{Ne}>\mathrm{Ss}>\mathrm{Bs}$ (Fig. 1, 2 and 3). In a comparative study on CODs, Noma and Nakayama (1976) (23) found the degrading activity of these enzymes for pure cholesterol in a buffer system followed a reverse sequence $\mathrm{Bs}>\mathrm{Ss}>\mathrm{Ne}$. These observations appear to indicate that the activity of a COD is greatly influenced by the nature of the reaction system. For example, cholesterol oxidase isolated from Ss was found to be much more active in a reaction system of supercritical fluid carbon-dioxide, than in a buffer or yolk system. In supercritical carbon dioxide $\left(100 \mathrm{bar}, 35^{\circ} \mathrm{C}\right), \mathrm{COD}$ from Ss demonstrated $100 \%$ cholesterol degradation within $1 \mathrm{~h}$ (25).

The 12-h incubation chosen to investigate the effect of temperature on cholesterol degrading ability by all four enzymes was based on data presented in initial incubation studies (Fig. 1- 3), which showed the majority of cholesterol being degraded in the first $12 \mathrm{~h}$ (Fig. 4). The effect of temperature on the enzyme activity after 12-h incubation differed greatly between enzyme types (interaction significant at p<0.001, Fig. 4). An attempt was made to fit the treatment variation to linear, cubic and quadratic relationships, but none of these relationships could adequately. account for all the variation among the treatment means (the lack of fit was significant, $\mathrm{p}<0.001$ ).

The most effective temperature for $\mathrm{Bs} C O D$ and that of the other CODs was around 37 and $45^{\circ} \mathrm{C}$, respectively. The enzymes isolated from $\mathrm{Pf}, \mathrm{Ne}$ and Ss showed good heat stability with substantial activity retained even at $60^{\circ} \mathrm{C}$. The Pf COD has an effective temperature range from 5 to $60^{\circ} \mathrm{C}$, and from 5 to $37^{\circ} \mathrm{C}$ it is clearly superior to the other three enzymes. 


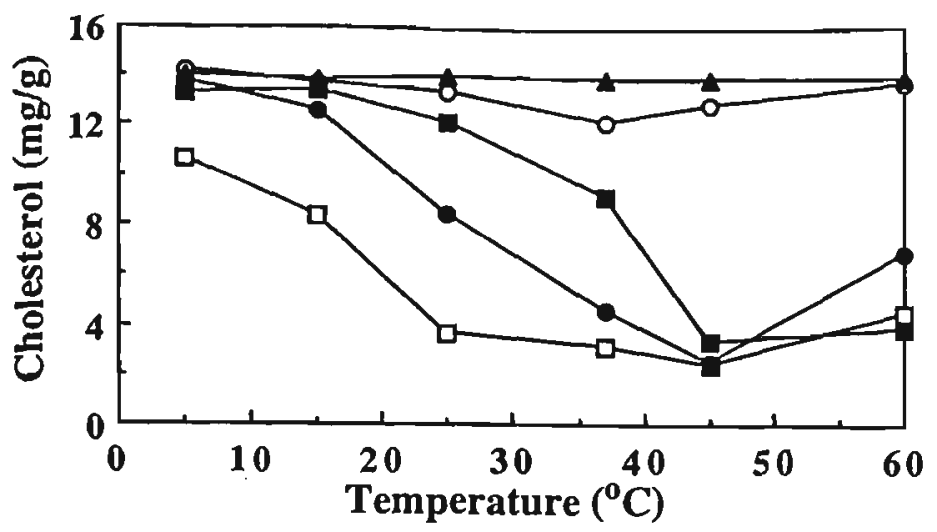

Figure 4. Effect of temperature on the activity of cholesterol oxidases from $\mathrm{Pf}(\square), \mathrm{Ne}(\mathrm{D})$, Ss (O) and Bs (O) in egg yolk, after $12 \mathrm{~h}$. $\mathrm{Mg} / \mathrm{g}$ residual cholesterol for control (4). LSD (for comparing the control treatments at different temperatures) $=0.58 . L S D$. (for all other comparisons) $=1.07$.

The most effective temperature/enzyme combination for maximum cholesterol degradation after $12 \mathrm{~h}$, was determined to be that by $\mathrm{Pf} C O D$ incubated at $37^{\circ} \mathrm{C}(\mathrm{LSD}=1.07$ $\mathrm{mg}$ cholesterol/g). Raising the temperature to $45^{\circ} \mathrm{C}$ did not produce a significant change in the amount of cholesterol degraded by this enzyme, and heating further to $60^{\circ} \mathrm{C}$ was no different to incubating at $25^{\circ} \mathrm{C}$ for the same period (LSD $=1.07 \mathrm{mg}$ cholesterol $/ \mathrm{g}$ ). At the low temperature of $5^{\circ} \mathrm{C}$, Pf COD was the only enzyme to achieve a cholesterol reduction (up to $28.7 \%$ ) which was significantly different to the control (LSD $=0.88 \mathrm{mg}$ cholesterol/g).

\section{Effect of concentration.}

The effect of enzyme concentration on cholesterol degradation is shown in Fig. 5. COD from Bs was not used due to the poor activity it demonstrated in the first two studies.

Based on data presented in Fig. 4, yolk samples were incubated at the optimum temperature of $45^{\circ} \mathrm{C}$. An incubation time of two hours was chosen because the data presented in Fig. 1 indicates that the reaction order followed first order kinetics for this initial period.

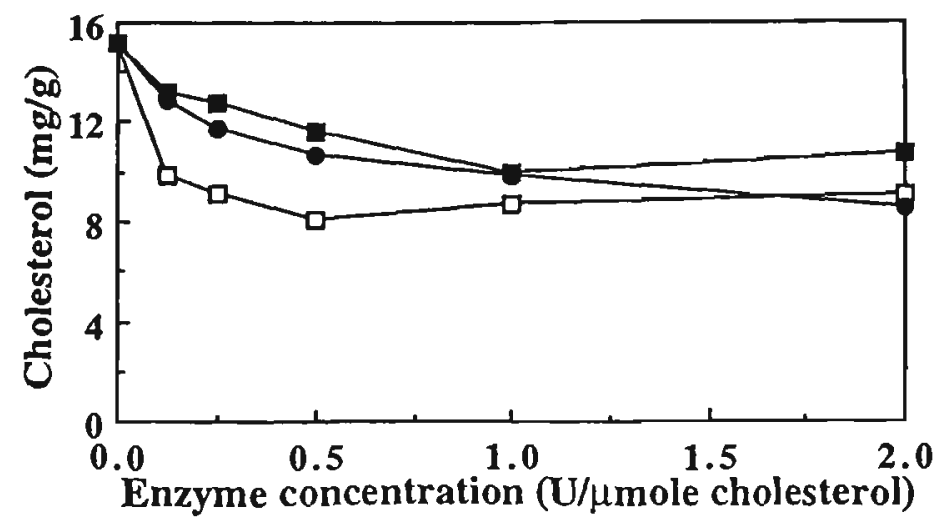

Figure 5. Effect of enzyme concentration on the degradation of cholesterol in egg yolk, at $45^{\circ} \mathrm{C}$ after $2 \mathrm{~h}$. Cholesterol oxidase from $P f(\square)$. Ne ( $\square$ ) and Ss (O). LSD (for comparing control with non-control treatments at different concentrations) $=0.41 . L S D$ (for all other comparisons) $=0.47$.
The effect of enzyme concentration on the degradation of egg yolk cholesterol after $2 \mathrm{~h}$ differed significantly (interaction significant as $\mathrm{p}<0.01$, Fig. 5). As in the previous study on temperature effect, an attempt was made to fit the treatment variation to linear, cubic and quadratic relationships, but none of these relationships could adequately account for all the variation among the treatment means (the lack of fit was significant, $\mathrm{p}<0.01$ ).

The most effective concentration of Pf $\mathrm{COD}$ and $\mathrm{Ne}$ COD was around $0.5 \mathrm{U} / \mu \mathrm{M}$ cholesterol and $1 \mathrm{U} / \mu \mathrm{M}$ cholesterol, respectively. Increasing the concentration of either of these enzymes further did not produce significantly different reductions in cholesterol (LSD $=0.47 \mathrm{mg}$ cholesterol/g). Streptomyces species COD showed significant changes in activity up to the maximum concentration of $2 \mathrm{U} / \mu \mathrm{M}$ cholesterol used in this investigation $(\mathrm{LSD}=0.47$ $\mathrm{mg}$ cholesterol/g). Surprisingly, even at the low concentration of $0.125 \mathrm{U} / \mu \mathrm{M}$ cholesterol, COD from Pf degraded more than one-third (up to $34.6 \%$ ) of the egg cholesterol in $2 \mathrm{~h}$. This was the only significantly different reduction between treatments seen at this concentration (LSD $=0.47$ $\mathrm{mg}$ cholesterol $/ \mathrm{g}$ ). Increasing the concentration of Pf COD further to 1 and $2 \mathrm{U} / \mu \mathrm{M}$ did not produce a reduction in cholesterol that was significantly different (LSD $=0.47 \mathrm{mg}$ cholesterol/g).

Cholesterol degradation was significantly different to that of the control at all concentrations and enzyme types (LSD $=0.41 \mathrm{mg}$ cholesterol/g). The most effective enzyme type/concentration combination was that of Pf COD at 0.5 $\mathrm{U} / \mu \mathrm{M}$.

\section{Cholesterol degradation products.}

A number of COD products have been detected in foods including spray-dried egg yolk powder (19). The adverse health effects of these oxidation products are still a topic for debate.

In this study, the degradation products of egg cholesterol were examined by TLC and confirmed by MSD. The only steroid-like component detected was 4-cholesten-3one, for each of the four enzymes. The 4-cholesten-3-one has been described as the first degradation product of cholesterol oxidase catalysis in species of Nocardia, Brevibacterium, Pseudomonas and Streptomyces by many investigators $(2,5,10,28,31,33)$. Aihara et al. (2) also found this compound in egg yolk inoculated with $R$. equi No. 23, a COD producing microorganism, but it was converted rapidly into non-steroid components.

\section{CONCLUSION}

The cholesterol degrading capacity of four COD enzymes in egg yolk, a complex food system, has been investigated. All enzymes catalyzed the transformation of cholesterol to 4-cholesten-3-one. More than 50\% of egg cholesterol could be reduced by three COD's (Ne, Pf and Ss), within $24 \mathrm{~h}$. The Pf COD was effective over a wide temperature range including at $4^{\circ} \mathrm{C}$. The low concentration of COD from Pf required to achieve significant reduction in yolk cholesterol coupled with the ability of this enzyme to degrade cholesterol at $4^{\circ} \mathrm{C}$ may make this enzyme, com- 
pared to the other three investigated, an economical and practical method for lowering the cholesterol level in egg yolk.

\section{ACKNOWLEDGMENTS}

We thank the Egg Industry Research Council for financial support, Kym Butler of the Victorian Institute of Animal Science for assistance with the design of our experiments and statistical analysis, Vince Murone of Victoria University of Technology for assistance with GC-MSD analysis, and Harry Laan, post-doctoral fellow from the University of Groningen in the Netherlands, for valuable discussion.

\section{REFERENCES}

1. Aihara, H., K. Watanabe and R. Nakamura. 1986. Characterization of production of cholesterol oxidase in three Rhodococcus strains. J. Appl. Bacteriol. 61:269-274.

2. Aihara, H., K. Watanabe and R. Nakamura. 1988. Degradation of cholesterol in egg yolk by Rhodococcus equi No. 23. J. Food Sci. 53:659-660.

3. Aihara, H., K. Watanabe and R. Nakamura. 1988. Degradation of cholesterol in hens egg yolk and its low density lipoproteins by extracellular enzymes of Rhodococcus equi No 23. Lebensum.Wiss.u-Technol 21:342-345.

4. Anonymous. 1991. A unique line: Liquid eggs in Tetra Povicvk packaging. Brot Backwaven 39:391.

5. Cheetham, P. S. J., P. Dunil and M. D. Lilly. 1982. The characterization and interconversion of three forms of cholesterol oxidase extracted from Nocardia rhodochrous. Biochemical J. 201:515-521.

6. Cheillan, F., H. Lafont, E. Termine, F. Fernandez, P. Sauve and G. Lesgards. 1989. Molecular characteristics of the cholesterol oxidase and factors influencing its activity. Biochemica et Biophysica Acta 999:233-238.

7. Christodoulou, S., T. V. Hung., M. A. Trewhella and R. Black. 1993. Enzymatic Degradation of Egg Cholesterol. 26th Convention Proceedings AIFST, 2nd-6th May, Adelaide.

8. Cully, J and H. R. Vollbrecht. 1992. Process for manufacture of cholesterol reduced egg yolk. Patent DE $4029287 \mathrm{Al}$.

9. Cully, J and H. R. Vollbrecht. 1991. Process for removal $\beta$ cyclodextrin from egg yolk. Patent DE $4001611 \mathrm{Cl}$.

10. Flegg, H. M. 1973. An investigation of the determination of serum cholesterol by an enzymatic method. Ann. Clin. Biochem. 10:79.

11. Flynn, M. A., Nolph, T. C. Flynn., R. Kahrs and G. Krause. 1979. Effect of dietary eggs on human serum cholesterol and triglycerides. Am. J. Clin. Nutr. 32:1051-1057.

12. Froning, G. W., R. L. Wehling, S. L. Cuppett, M. M. Pierce, L. Niemann and D. K. Siekman. 1990. Extraction of cholesterol and other lipids from dried egg yolk using supercritical carbon dioxide. J. Food Sci. 55:95-98.

13. Hargis, P. S. 1988. Modifying egg yolk cholesterol in the domestic fowl. Worlds Poultry Sci. J. 44:17-24.

14. Hung, T. V. and M. Unger. 1993. Production of cholesterol reduced egg products by supercritical fluid extraction AIFST 26th Convention Proceedings, 2nd-6th May, Adelaide.
15. Jellema, A. 1980. The action of milk lipase on milkfat globules: Physiological and mechanical factors. Neth. Milk Dairy J. 34:133135.

16. Johnson, T. L. and G. A. Somkuti. 1990. Properties of cholesterol dissimilation by Rhodococcus equi. J. Food Prot. 53:332-335.

17. Kern, F., Jr. 1991. Normal plasma cholesterol in 88 year old man who eats 25 eggs per day. N. Engl. J. Med. 324:896-899.

18. Kovacs, M. I. P. 1990. Determination of cholesterol in pasta products using gas-liquid chromatography. J. Cereal Sci. 11:291-297.

19. Kumar, N. and O. P. Singhal. 1991. Cholesterol oxides and atherosclerosis: A Review. J. Sci. Food Agric. 55:497-510.

20. Larsen, J. E. and G. W. Froning. 1981. Extraction and processing of various components from egg yolk. Poult. Sci. 60:160-167.

21. Lebovics, V. K, O. Gaal, L. Somogyi and J. Farkas. 1992. Cholesterol oxides in $\gamma$-irradiated spray-dried egg powder. J. Sci. Food Agric. 60:251-254.

22. Naber, E. C. and D. B. Mathew. 1985. Analysis for generation of cholesterol oxidation products in egg yolk by heat treatment. Poult. Sci. 64:341-347.

23. Noma, A. and K. Nakayama. 1976. Comparative studies on cholesterol oxidases from different sources. Clinica Chimica Acta 73:487496.

24. Owen, R., W., Mason, A. N. Mason and R. F. Billton. 1983. The degradation of cholesterol by Pseudomonas sp. NCIB 10590 under aerobic conditions. J. Lipid Research 24:1500-1511.

25. Randolph, T. W., D. S. Clark, H. Blanch and Prausniz. 1988 Enzymatic oxidation of cholesterol aggregates in supercritical carbon dioxide. Science 238:387-390.

26. Reddy, R. V., S. F. Lightsey and D. V. Maurice. 1991. Effect of feeding garlic oil on performance and egg yolk cholesterol concentration. Poult. Sci. 70:2006-2009.

27. Sheather, K. R. 1992. Successes and failures in Australia. Poultry Intemational 31:60-68.

28. Smith A. G. and C. J. Brooks. 1974. Application of cholesterol oxidase in the analysis of steroids. J. Chromatography $101373-378$.

29. Snedecor, G. W. and W. G. Cochran. 1967. Statistical Methods. Sixth ed. Iowa State University Press, Ames, LA.

30. Stehbens, W. E. 1990. The Cholesterol-Hear Disease Controversy. ASEAN Food J. 5:131-141.

31. Tomioka, H., M. Kagawa and S. Nakamura. 1976. Some enzymatic properties of $3 \boldsymbol{\beta}$-hydroxysteroid oxidase produced by Strepiomjces violascens. J. Biochem 79:903-915.

32. Trustwell, A. S. 1978. Diet and plasma lipids-a reappraisal. Am. J. Clin. Nutr. 31:977-987.

33. Uwajima, T., H. Yagi and O. Terada. 1974. Properties of crystalline $3 \beta$-hydroxysteroid oxidase of Brevibacterium sterolicum. Agric. Biol. Chem 38:1149-1156.

34. Vorster, H. H., A. J. Spinnler Benade, H. C. Bamard, M. M. Locke. N. Silvis, C. S. Venter, C. M. Smuts, G. P. Engelbrecht and M. P. Marais. 1992. Egg intake does not change plasma lipoprotein and coagulation profiles. Am. J. Clin. Nutr. 55:400-410.

35. Watkins, B. A. and R. G. Elkin. 1992. Dietary modulatisn of oleic and stearic acids in egg yolk. J. Food Comp. Analysis 5:2 $2,9-215$.

36. Xiansheng W., T. V. Hung. P. G. Drew and K. Versteeg. 1990 Enzymatic degradation of cholesterol in milk. Aust. J. Dairy Tech. 45(2):50-52. 